\title{
Palladium-Catalyzed Intramolecular Asymmetric Hydroamination, Hydroalkoxylation and Hydrocarbonation of Alkynes
}

Nitin T. Patil, Léopold Mpaka Lutete, Huanyou Wu, Nirmal K. Pahadi, Ilya D. Gridnev, and Yoshinori Yamamoto*

Department of Chemistry, Graduate School of Science, Tohoku University, Sendai 980-8578, Japan

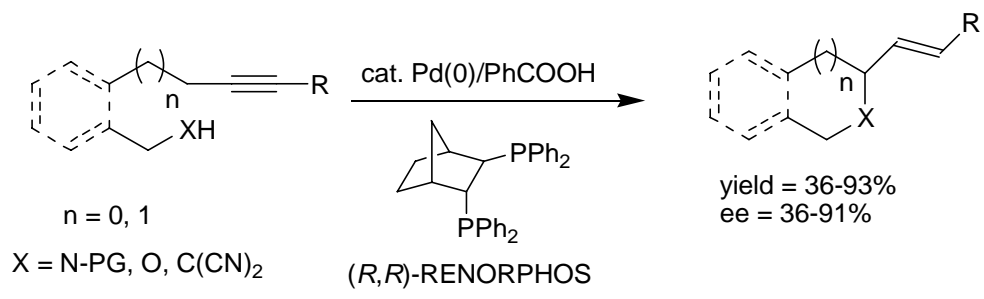

Page S-1: Title of the paper, author's name and address along with the contents.

Page S-2: General procedures

Page S-3: Catalytic Asymmetric Hydroamination of Alkynes

Page S-6: Catalytic Asymmetric Hydroalkoxylation of Alkynes

Page S-11: Catalytic Asymmetric Hydrocarbonation of Alkynes

Page S-15: ${ }^{1} \mathrm{H}$ NMR spectra of all compounds

Page S-50: Cartesian Coordinates 


\section{General}

The ${ }^{1} \mathrm{H}$ NMR and ${ }^{13} \mathrm{C}$ NMR spectra were recorded at $400 \mathrm{MHz}$ and $100 \mathrm{MHz}$, respectively, using $\mathrm{CDCl}_{3}$ as solvent and were reported in ppm relative to $\mathrm{CHCl}_{3}(\delta=7.26)$ for ${ }^{1} \mathrm{H}$ NMR and relative to the central $\mathrm{CDCl}_{3}(\delta=77.00)$ resonance for ${ }^{13} \mathrm{C}$ NMR. Coupling constants in ${ }^{1} \mathrm{H}$ NMR are in Hz. Optical Rotations were measured on a digital polarimeter. HPLC was performed using a CHIRALCEL OD column (4.6 mm x $250 \mathrm{~mm}) \cdot \mathrm{Pd}_{2} \mathrm{dba}_{3} \cdot \mathrm{CHCl}_{3}$ and benzoic acid were purchased. $(R, R)$-NORPHOS, biphenyl ligands 16, 17 and 18 were purchased. $(R, R)$-RENORPHOS 4 was prepared from commercial available $(R, R)$-NORPHOS by hydrogenation with $10 \% \mathrm{Pd} / \mathrm{C}$ in methanol (see ref 35 in the text). Unless otherwise noted all experiments were carried out using $(R, R)$-RENORPHOS.

General procedures for asymmetric intramolecular hydroamination, hydroalkoxylation and hydrocarbonation reactions

Conditions A: To $\mathrm{Pd}_{2}(\mathrm{dba})_{3} \cdot \mathrm{CHCl}_{3}(5 \mathrm{~mol} \%), \operatorname{PhCOOH}(10 \mathrm{~mol} \%)$ and $(R, R)-\mathrm{RENORPHOS}^{35}(25$ mol\%) was added a substrate $(0.125 \mathrm{mmol})$ in $1.5 \mathrm{ml}$ of benzene under Ar atmosphere in a screw capped vial. After heating at $100^{\circ} \mathrm{C}$ for $72 \mathrm{~h}$, the reaction mixture was filtered through a short silica gel column using diethyl ether as an eluent. After evaporation of the solvent, the residue was purified by silica gel column chromatography to afford the corresponding cyclization product.

Conditions B: To $\mathrm{Pd}_{2}(\mathrm{dba})_{3} \cdot \mathrm{CHCl}_{3}(20 \mathrm{~mol} \%)$, $\mathrm{PhCOOH}(40 \mathrm{~mol} \%)$ and $(R, R)-\mathrm{RENORPHOS}(100$ mol\%) was added a substrate $(0.125 \mathrm{mmol})$ in $1.5 \mathrm{ml}$ of benzene/hexane $(2: 1)$ under Ar atmosphere in a screw capped vial. After heating at $80^{\circ} \mathrm{C}$ for $72 \mathrm{~h}$, the reaction mixture was filtered through a short silica gel column using diethyl ether as an eluent. After evaporation of the solvent, the residue was purified by silica gel column chromatography to afford the corresponding cyclization product.

Conditions C: To $\mathrm{Pd}_{2}(\mathrm{dba})_{3} \cdot \mathrm{CHCl}_{3}(10 \mathrm{~mol} \%)$, PhCOOH $(20 \mathrm{~mol} \%)$ and $(R, R)-\mathrm{RENORPHOS}(60$ mol\%) was added a substrate $(0.125 \mathrm{mmol})$ in benzene $(0.5 \mathrm{M})$ under Ar atmosphere in a screw capped 
vial. After heating at $100^{\circ} \mathrm{C}$ for $72 \mathrm{~h}$, the reaction mixture was filtered through a short silica gel column using diethyl ether as an eluent. After evaporation of the solvent, the residue was purified by silica gel column chromatography to afford the corresponding cyclization product.

\section{Catalytic Asymmetric Hydroamination of Alkynes}

For detail characterization, see our previously reported communications, ref 19 in text.

We have screened several nonracemic chiral ligands for the intramolecular hydroamination reaction, the results are listed below.

\section{Ligand Screened}




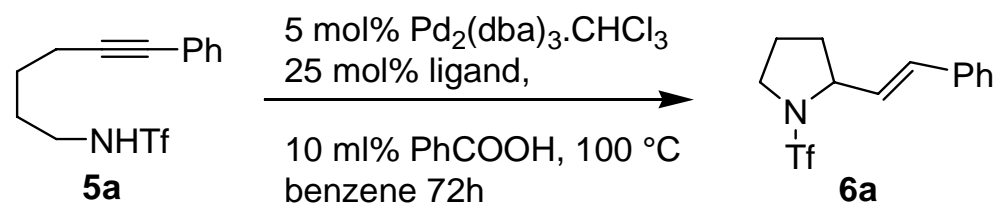

\begin{tabular}{|l|l|l|l|}
\hline \multicolumn{1}{|c|}{ Entry } & \multicolumn{1}{|c|}{ L $^{*}$} & \multicolumn{1}{c|}{ Yield (\%) } & \multicolumn{1}{c|}{ Ee (\%) } \\
\hline 1 & (S)-QUINAP (I) & 59 & 10 \\
\hline 2 & (R)-BINAP (II) & 67 & 8 \\
\hline 3 & (R)-MOP (III) & 89 & 6 \\
\hline 4 & Trost Ligand (1) & 51 & 12 \\
\hline 5 & (R, R)-Me-BPE (IV) & No Reaction & - \\
\hline 6 & (R, R)-Me-DUPHOS (V) & No Reaction & - \\
\hline 7 & (S, S)-Me-FerroTane (VI) & 57 & 3 \\
\hline 8 & (S)-PHANEPHOS (VII) & 76 & 8 \\
\hline 9 & (S, S)-CHIRAPHOS (2) & 92 & 25 \\
\hline 10 & (R, R)-NORPHOS (3) & 5 & 98 \\
\hline 11 & (R, R)-RENORPHOS (4) & 54 & 81 \\
\hline 12 & (S, S)-BDPP (VIII) & 70 & 15 \\
\hline 13 & (R)-PROPHOS (IX) & 61 & 0 \\
\hline 14 & (S, S)-DIOP (X) & 92 & 6 \\
\hline 15 & (S, S)-BPPM (XI) & 81 & 6 \\
\hline 16 & (+) NMDPP (XII) & 81 & 5 \\
\hline 17 & (R)-(S)-JOSIPHOS (XIII) & 77 & 7 \\
\hline 18 & (R)-(S)-BPPFOAc (XIV) & 65 & 4 \\
\hline 19 & (XV) & 91 & 0 \\
\hline 20 & (XVI) & 89 & 0 \\
\hline 21 & (XVII) & 41 & 6 \\
\hline
\end{tabular}




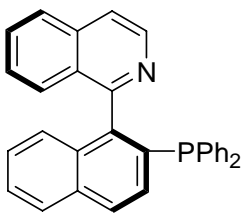

(S)-QUINAP (I)

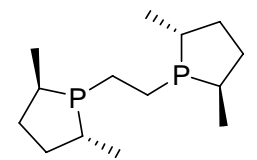

(R, R)-Me-BPE (IV)

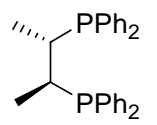

(S, S)-CHIRAPHOS (2)

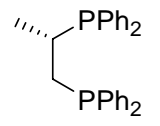

(R)-PROPHOS (IX)

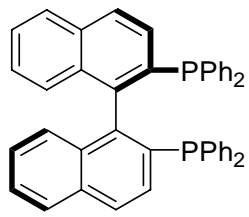

(R)-BINAP (II)

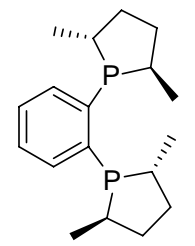

(R, R)-Me-DUPHOS (V)

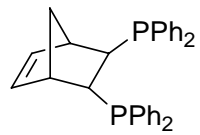

(R, R)-NORPHOS (3)

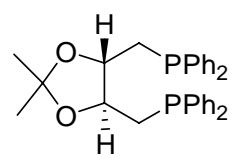

$(\mathrm{S}, \mathrm{S})$-DIOP $(\mathbf{X})$

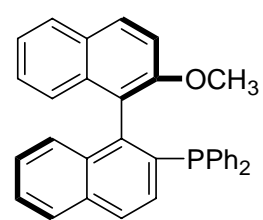

(R)-MOP (III)

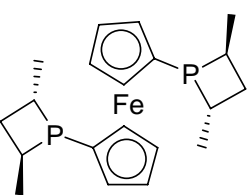

(S, S)-Me-FerroTane (VI)

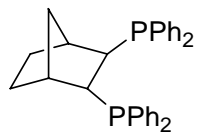

(R, R)-RENORPHOS (4)

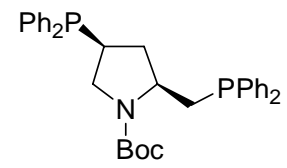

(S, S)-BPPM (XI)

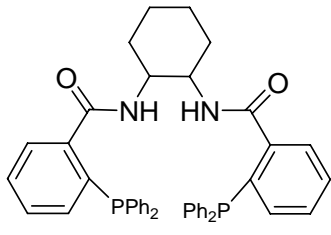

Trost Ligand (1)

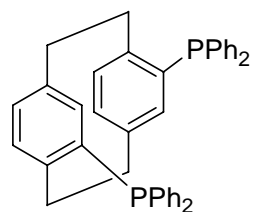

(S)-PHANEPHOS (VII)

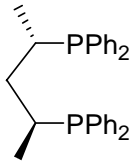

(S, S)-BDPP (VIII)

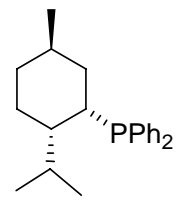

(+) NMDPP (XII)

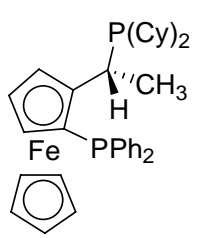

(R)-(S)-JOSIPHOS (XIII)

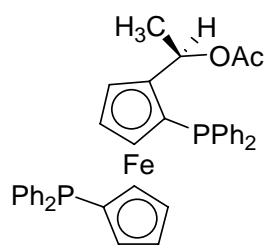

(R)-(S)-BPPFOAc (XIV)

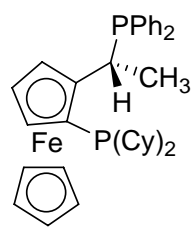

$(\mathbf{X V})$

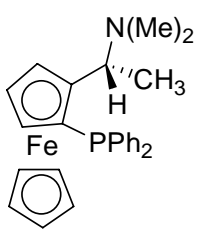

(XVI)

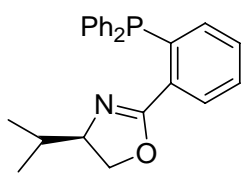

(XVII) 
Preparation of Substrates: The substrates were prepared by the Sonogashira reactions of terminal alkynes with corresponding iodo compounds.

\section{General procedure for Sonogashira reaction:}

To a mixture of iodobenzenes (1 equiv), $\mathrm{Pd}\left(\mathrm{PPh}_{3}\right)_{4}(1 \mathrm{~mol} \%)$, and $\mathrm{CuI}(2 \mathrm{~mol} \%)$ in $\mathrm{Et}_{3} \mathrm{~N}$ were added alkynes ( 1 equiv) at room temperature under an argon atmosphere. The mixture was stirred at room temperature overnight. After the reaction was completed, $\mathrm{NH}_{3} / \mathrm{NH}_{4} \mathrm{Cl}$ aqueous solution was added and the product was extracted with ether. Then the organic phase was dried with $\mathrm{Na}_{2} \mathrm{SO}_{4}$ and concentrated. The residue was purified by column chromatography ( silica gel, hexane/AcOEt $=4 / 1$ ) to give corresponding alkynols 7a-i in high yields.
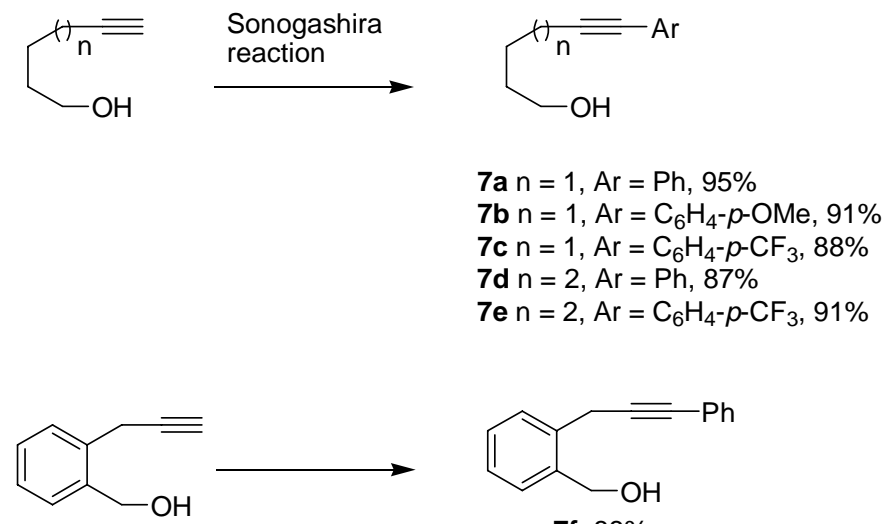

$$
\text { 7f, } 88 \%
$$
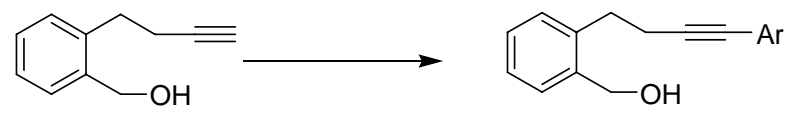

$7 \mathrm{~g} \mathrm{Ar}=\mathrm{Ph}, 88 \%$

7h $\mathrm{Ar}=\mathrm{C}_{6} \mathrm{H}_{4}-p-\mathrm{CF}_{3}, 82 \%$

$7 \mathbf{i} \mathrm{Ar}=\mathrm{C}_{6} \mathrm{H}_{4}-p-\mathrm{OMe}, 87 \%$

7a. ${ }^{1} \mathrm{H}$ NMR $\left(\mathrm{CDCl}_{3}, 400 \mathrm{MHz}\right) \delta$ 7.40-7.37 (m, 2H), 7.29-7.25 (m, 3H), $3.70(\mathrm{t}, J=6.4 \mathrm{~Hz}, 2 \mathrm{H}), 2.45$ $\left.(\mathrm{t}, J=6.4 \mathrm{~Hz}, 2 \mathrm{H}), 1.77-1.66(\mathrm{~m}, 5 \mathrm{H}) ;{ }^{13} \mathrm{C} \mathrm{NMR}\left(\mathrm{CDCl}_{3}, 100 \mathrm{MHz}\right)\right) \delta 131.4,128.1,127.5,123.8$, 89.8, 80.9, 62.4, 31.9, 25.0, 19.2; HRMS calcd for $\mathrm{C}_{12} \mathrm{H}_{14} \mathrm{O}\left(\mathrm{M}^{+}\right)$174.1045, found $174.1039\left(\mathrm{M}^{+}\right)$.

7b. ${ }^{1} \mathrm{H} \mathrm{NMR}\left(\mathrm{CDCl}_{3}, 400 \mathrm{MHz}\right) \delta 7.31(\mathrm{~d}, J=8.8 \mathrm{~Hz}, 2 \mathrm{H}), 6.80(\mathrm{~d}, J=8.8 \mathrm{~Hz}, 2 \mathrm{H}), 3.79(\mathrm{~s}, 3 \mathrm{H}), 3.70$ $(\mathrm{t}, J=6.8 \mathrm{~Hz}, 2 \mathrm{H}), 2.44(\mathrm{t}, J=6.8 \mathrm{~Hz}, 2 \mathrm{H}), 1.75-1.67(\mathrm{~m}, 4 \mathrm{H}), 1.58(\mathrm{bs}, 1 \mathrm{H}) ;{ }^{13} \mathrm{C} \mathrm{NMR}\left(\mathrm{CDCl}_{3}, 100\right.$ 
MHz) ) $\delta 158.9,132.7,115.9,113.7,88.2,80.6,62.5,55.3,32.0,25.1,19.2$; HRMS calcd for $\mathrm{C}_{13} \mathrm{H}_{16} \mathrm{O}_{2}$ $\left(\mathrm{M}^{+}\right)$204.1150, found 227.1043 (M+Na).

7c. ${ }^{1} \mathrm{H}$ NMR $\left(\mathrm{CDCl}_{3}, 400 \mathrm{MHz}\right) \delta 7.52(\mathrm{~d}, J=8.4 \mathrm{~Hz}, 2 \mathrm{H}), 7.47(\mathrm{~d}, J=8.4 \mathrm{~Hz}, 2 \mathrm{H}), 3.71(\mathrm{t}, J=6.8$ $\mathrm{Hz}, 2 \mathrm{H}), 2.47(\mathrm{t}, J=6.8 \mathrm{~Hz}, 2 \mathrm{H}), 1.76-1.69(\mathrm{~m}, 4 \mathrm{H}), 1.54(\mathrm{bs}, 1 \mathrm{H}) ;{ }^{13} \mathrm{C} \mathrm{NMR}\left(\mathrm{CDCl}_{3}, 100 \mathrm{MHz}\right) \delta$ 131.7, 129.4, 127.7, 125.0, 122.6, 92.7, 79.8, 62.4, 31.9, 24.9, 19.3; HRMS calcd for $\mathrm{C}_{13} \mathrm{H}_{13} \mathrm{~F}_{3} \mathrm{O}\left(\mathrm{M}^{+}\right)$ 242.0918, found 265.0811 (M+Na).

7d. ${ }^{1} \mathrm{H}$ NMR $\left(\mathrm{CDCl}_{3}, 400 \mathrm{MHz}\right) \delta$ 7.40-7.36 (m, 2H), 7.29-7.25 (m, 3H), 3.67 (t, $\left.J=6.8 \mathrm{~Hz}, 2 \mathrm{H}\right), 2.42$ (t, $J=6.8 \mathrm{~Hz}, 2 \mathrm{H}), 1.68-1.50(\mathrm{~m}, 6 \mathrm{H}), 1.48(\mathrm{bs}, 1 \mathrm{H}) ;{ }^{13} \mathrm{C} \mathrm{NMR}\left(\mathrm{CDCl}_{3}, 100 \mathrm{MHz}\right) \delta 131.4,128.1$, 127.4, 123.9, 90.0, 80.7, 62.8, 32.3, 28.5, 25.1, 19.4; HRMS calcd for $\mathrm{C}_{13} \mathrm{H}_{16} \mathrm{O}\left(\mathrm{M}^{+}\right)$188.1201, found $211.1093(\mathrm{M}+\mathrm{Na})$.

7e. ${ }^{1} \mathrm{H}$ NMR $\left(\mathrm{CDCl}_{3}, 400 \mathrm{MHz}\right) \delta 7.52(\mathrm{~d}, J=8.4 \mathrm{~Hz}, 2 \mathrm{H}), 7.46(\mathrm{~d}, J=8.4 \mathrm{~Hz}, 2 \mathrm{H}), 3.67(\mathrm{t}, J=6.8$ $\mathrm{Hz}, 2 \mathrm{H}), 2.44(\mathrm{t}, J=6.8 \mathrm{~Hz}, 2 \mathrm{H}), 1.75-1.49(\mathrm{~m}, 7 \mathrm{H}) ;{ }^{13} \mathrm{C} \mathrm{NMR}\left(\mathrm{CDCl}_{3}, 100 \mathrm{MHz}\right) \delta 131.6,129.3$, 125.3, 124.9, 92.9, 79.6, 62.7, 32.2, 28.4, 25.1, 19.4; HRMS calcd for $\mathrm{C}_{14} \mathrm{H}_{15} \mathrm{~F}_{3} \mathrm{O}\left(\mathrm{M}^{+}\right)$256.1075, found $279.0967(\mathrm{M}+\mathrm{Na})$.

7f. ${ }^{1} \mathrm{H}$ NMR $\left(\mathrm{CDCl}_{3}, 400 \mathrm{MHz}\right) \delta$ 7.53-7.26 (m, 9H), 4.79 (s, 2H), $3.88(\mathrm{~s}, 2 \mathrm{H}), 1.50$ (bs, $\left.1 \mathrm{H}\right) ;{ }^{13} \mathrm{C}$ $\mathrm{NMR}\left(\mathrm{CDCl}_{3}, 100 \mathrm{MHz}\right) \delta 138.1,134.9,131.5,129.0,128.3,128.2,128.1,127.8,127.2,123.3,87.3$, 82.9, 63.3, 23.1; HRMS calcd for $\mathrm{C}_{16} \mathrm{H}_{14} \mathrm{O}\left(\mathrm{M}^{+}\right)$222.1045, found $245.0937(\mathrm{M}+\mathrm{Na})$.

7g. ${ }^{1} \mathrm{H}$ NMR ( $\left.\mathrm{CDCl}_{3}, 400 \mathrm{MHz}\right) \delta$ 7.36-7.22 (m, 9H), $4.73(\mathrm{~s}, 2 \mathrm{H}), 2.99$ (t, $\left.J=6.8 \mathrm{~Hz}, 2 \mathrm{H}\right), 2.71$ (t, $J=$ $6.8 \mathrm{~Hz}, 2 \mathrm{H}), 1.99$ (bs, $1 \mathrm{H}) ;{ }^{13} \mathrm{C} \mathrm{NMR}\left(\mathrm{CDCl}_{3}, 100 \mathrm{MHz}\right) \delta 138.8,138.4,131.4,129.6,128.5,128.1$, 128.0, 127.6, 126.7, 123.5, 89.4, 81.5, 63.2, 31.4, 21.4; HRMS calcd for $\mathrm{C}_{17} \mathrm{H}_{16} \mathrm{O}\left(\mathrm{M}^{+}\right)$236.1201, found $259.1093(\mathrm{M}+\mathrm{Na})$.

7h. ${ }^{1} \mathrm{H}$ NMR $\left(\mathrm{CDCl}_{3}, 400 \mathrm{MHz}\right) \delta 7.51(\mathrm{~d}, J=8.0 \mathrm{~Hz}, 2 \mathrm{H}), 7.43(\mathrm{~d}, J=8.0 \mathrm{~Hz}, 2 \mathrm{H}), 7.37(\mathrm{~d}, J=6.8$ Hz, 1H), 7.30-7.24 (m, 3H), 4.77 (s, 2H), 3.03 (t, $J=7.2 \mathrm{~Hz}, 2 \mathrm{H}$ ), 2.75 (t, $J=7.2 \mathrm{~Hz}, 2 \mathrm{H}$ ), 1.69 (bs, $1 \mathrm{H}) ;{ }^{13} \mathrm{C} \mathrm{NMR}\left(\mathrm{CDCl}_{3}, 100 \mathrm{MHz}\right) \delta 138.7,138.3,131.6,129.7,129.5,128.5,128.1,127.4,126.8$, 125.2, 125.0, 125.0, 92.2, 80.4, 63.4, 31.2, 21.4; HRMS calcd for $\mathrm{C}_{18} \mathrm{H}_{15} \mathrm{~F}_{3} \mathrm{O}\left(\mathrm{M}^{+}\right)$304.1075, found $304.1070\left(\mathrm{M}^{+}\right)$. 
7i. ${ }^{1} \mathrm{H}$ NMR $\left(\mathrm{CDCl}_{3}, 400 \mathrm{MHz}\right) \delta 7.37(\mathrm{~d}, J=6.8 \mathrm{~Hz}, 1 \mathrm{H}), 7.30-7.21(\mathrm{~m}, 5 \mathrm{H}), 6.79(\mathrm{~d}, J=8.8 \mathrm{~Hz}, 2 \mathrm{H})$, 4.77 (s, 2H), 3.79 (s, 3H), 3.01 (t, $J=7.2 \mathrm{~Hz}, 2 \mathrm{H}), 2.71(\mathrm{t}, J=7.2 \mathrm{~Hz}, 2 \mathrm{H}), 1.51(\mathrm{bs}, 1 \mathrm{H}) ;{ }^{13} \mathrm{C} \mathrm{NMR}$ $\left(\mathrm{CDCl}_{3}, 100 \mathrm{MHz}\right) \delta 159.0,138.9,138.4,132.8,129.6,128.6,128.0,126.6,115.7,113.8,87.8,81.2$, 63.3, 55.3, 31.5, 21.5; HRMS calcd for $\mathrm{C}_{18} \mathrm{H}_{18} \mathrm{O}_{2}\left(\mathrm{M}^{+}\right)$266.1307, found $289.1301(\mathrm{M}+\mathrm{Na})$.

\section{Catalytic Asymmetric Cyclization or alkynols 7:}

The alkynols 7a-i were subjected to palladium catalysis under condition $\mathbf{C}$ to give corresponding oxygen heterocycles 8a-i. Refer Table 3 in the text.

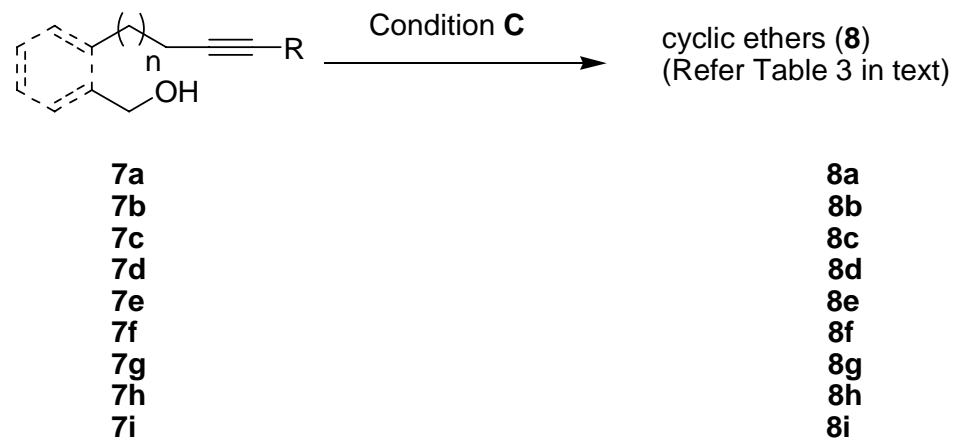

8a. ${ }^{1} \mathrm{H}$ NMR $\left(\mathrm{CDCl}_{3}, 400 \mathrm{MHz}\right) \delta$ 7.39-7.20 (m, $\left.5 \mathrm{H}\right), 6.58(\mathrm{~d}, J=16.0 \mathrm{~Hz}, 1 \mathrm{H}), 6.20(\mathrm{dd}, J=16.0,8.4$ $\mathrm{Hz}, 1 \mathrm{H}), 4.47$ (q, $J=8.4 \mathrm{~Hz}, 1 \mathrm{H}), 3.97(\mathrm{q}, J=8.4 \mathrm{~Hz}, 1 \mathrm{H}), 3.84(\mathrm{dt}, J=12.0,8.4 \mathrm{~Hz}, 1 \mathrm{H}), 2.17-2.09$ (m, 1H), 2.01-1.91 (m, 2H), 1.76-1.67 (m, 1H); ${ }^{13} \mathrm{C} \mathrm{NMR}\left(\mathrm{CDCl}_{3}, 100 \mathrm{MHz}\right) \delta 136.8,130.5,130.3$, 128.4, 127.4, 126.4, 79.6, 68.2, 32.4, 25.9; HRMS calcd for $\mathrm{C}_{12} \mathrm{H}_{14} \mathrm{O}\left(\mathrm{M}^{+}\right)$174.1045, found 174.1039 $\left(\mathrm{M}^{+}\right) ;[\alpha]^{23}=-14.1\left(\right.$ c $\left.0.5, \mathrm{CHCl}_{3}\right) ;$ ee $=80 \%$.

8b. ${ }^{1} \mathrm{H}$ NMR $\left(\mathrm{CDCl}_{3}, 400 \mathrm{MHz}\right) \delta 7.30(\mathrm{~d}, J=8.4 \mathrm{~Hz}, 2 \mathrm{H}), 6.82(\mathrm{~d}, J=8.4 \mathrm{~Hz}, 2 \mathrm{H}), 6.51(\mathrm{~d}, J=16.0$ $\mathrm{Hz}, 1 \mathrm{H}), 6.05$ (dd, $J=16.0,6.8 \mathrm{~Hz}, 1 \mathrm{H}), 4.43$ (q, $J=7.6 \mathrm{~Hz}, 1 \mathrm{H}), 3.96$ (q, $J=7.6 \mathrm{~Hz}, 1 \mathrm{H}), 3.83$ (dt, $J=$ 12.0, 7.6 Hz, 1H), 3.79 (s, 3H), 2.16-2.06 (m, 1H), 2.01-1.89 (m, 2H), 1.74-1.65 (m, 1H); ${ }^{13} \mathrm{C}$ NMR $\left(\mathrm{CDCl}_{3}, 100 \mathrm{MHz}\right) \delta 159.0,130.0,129.6,128.2,127.5,113.9,79.9,68.1,55.3,32.5,25.9$; HRMS calcd for $\mathrm{C}_{13} \mathrm{H}_{16} \mathrm{O}_{2}\left(\mathrm{M}^{+}\right)$204.1150, found 227.1043 (M+Na); $[\alpha]^{15}=-10.8\left(c 1.0, \mathrm{CHCl}_{3}\right)$; ee $=40 \%$. 
8c. ${ }^{1} \mathrm{H}$ NMR $\left(\mathrm{CDCl}_{3}, 400 \mathrm{MHz}\right) \delta 7.54(\mathrm{~d}, J=8.0 \mathrm{~Hz}, 2 \mathrm{H}), 7.45(\mathrm{~d}, J=8.0 \mathrm{~Hz}, 2 \mathrm{H}), 6.61(\mathrm{~d}, J=16.0$ Hz, 1H), 6.30 (dd, $J=16.0,6.4 \mathrm{~Hz}, 1 \mathrm{H}), 4.49$ (q, $J=6.4 \mathrm{~Hz}, 1 \mathrm{H}), 3.97$ (q, $J=6.4 \mathrm{~Hz}, 1 \mathrm{H}), 3.85$ (dt, $J=$ 12.0, $6.4 \mathrm{~Hz}, 1 \mathrm{H}), 2.19-2.11(\mathrm{~m}, 1 \mathrm{H}), 2.05-1.90(\mathrm{~m}, 2 \mathrm{H}), 1.77-1.67(\mathrm{~m}, 1 \mathrm{H}) ;{ }^{13} \mathrm{C} \mathrm{NMR}\left(\mathrm{CDCl}_{3}, 100\right.$ MHz) $\delta 140.3,133.3,129.0,128.7,126.5,125.4,79.2,68.3,32.4,25.9$; HRMS calcd for $\mathrm{C}_{13} \mathrm{H}_{13} \mathrm{~F}_{3} \mathrm{O}$ $\left(\mathrm{M}^{+}\right) 242.0918$, found $281.0760(\mathrm{M}+\mathrm{O}+\mathrm{Na}) ;[\alpha]^{15}{ }_{\mathrm{D}}=-10.8\left(c 1.0, \mathrm{CHCl}_{3}\right)$; ee $=82 \%$.

8d. ${ }^{1} \mathrm{H}$ NMR $\left(\mathrm{CDCl}_{3}, 400 \mathrm{MHz}\right) \delta 7.31-7.10(\mathrm{~m}, 5 \mathrm{H}), 6.50(\mathrm{~d}, J=16.4 \mathrm{~Hz}, 1 \mathrm{H}), 6.13(\mathrm{dd}, J=16.4,6.0$ $\mathrm{Hz}, 1 \mathrm{H}), 4.01-3.98(\mathrm{~m}, 1 \mathrm{H}), 3.92-3.87(\mathrm{~m}, 1 \mathrm{H}), 3.47$ (dt, $J=11.6,2.8 \mathrm{~Hz}, 1 \mathrm{H}), 1.90-1.85(\mathrm{~m}, 1 \mathrm{H})$, 1.80-1.30 (m, 5H); ${ }^{13} \mathrm{C} \mathrm{NMR}\left(\mathrm{CDCl}_{3}, 100 \mathrm{MHz}\right) \delta 136.9,130.8,129.7,128.4,127.3,126.3,78.0,68.4$, 32.3, 25.9, 23.5; HRMS calcd for $\mathrm{C}_{13} \mathrm{H}_{16} \mathrm{O}\left(\mathrm{M}^{+}\right)$188.1201, found $188.1196\left(\mathrm{M}^{+}\right) ;[\alpha]^{22}{ }_{\mathrm{D}}=-13.6($ c 0.9 , $\left.\mathrm{CHCl}_{3}\right) ; \mathrm{ee}=78 \%$.

8e. ${ }^{1} \mathrm{H}$ NMR $\left(\mathrm{CDCl}_{3}, 400 \mathrm{MHz}\right) \delta 7.53(\mathrm{~d}, J=8.0 \mathrm{~Hz}, 2 \mathrm{H}), 7.45(\mathrm{~d}, J=8.0 \mathrm{~Hz}, 2 \mathrm{H}), 6.61(\mathrm{~d}, J=16.0$ $\mathrm{Hz}, 1 \mathrm{H}), 6.29(\mathrm{~d}, J=16.0,5.6 \mathrm{~Hz}, 1 \mathrm{H}), 4.09-4.06(\mathrm{~m}, 1 \mathrm{H}), 4.01-3.97(\mathrm{~m}, 1 \mathrm{H}), 3.58-3.51(\mathrm{~m}, 1 \mathrm{H}), 1.95-$ $1.85(\mathrm{~m}, 1 \mathrm{H}), 1.79-1.71(\mathrm{~m}, 1 \mathrm{H}), 1.66-1.42(\mathrm{~m}, 4 \mathrm{H}) ;{ }^{13} \mathrm{C} \mathrm{NMR}\left(\mathrm{CDCl}_{3}, 100 \mathrm{MHz}\right) \delta 140.5,133.4$, 129.3, 128.9, 126.4, 125.4, 122.8, 77.6, 68.5, 32.2, 25.9, 23.5; HRMS calcd for $\mathrm{C}_{14} \mathrm{H}_{15} \mathrm{~F}_{3} \mathrm{O}\left(\mathrm{M}^{+}\right)$ 256.1075 , found $256.1000\left(\mathrm{M}^{+}\right) ;[\alpha]^{16}=-18.8\left(c 0.9, \mathrm{CHCl}_{3}\right)$; ee $=74 \%$.

8f. ${ }^{1} \mathrm{H}$ NMR $\left(\mathrm{CDCl}_{3}, 400 \mathrm{MHz}\right) \delta$ 7.42-7.17 (m, 9H), $6.74(\mathrm{~d}, J=16.0 \mathrm{~Hz}, 1 \mathrm{H}), 6.28(\mathrm{dd}, J=16.0,8.0$ $\mathrm{Hz}, 1 \mathrm{H}), 5.76(\mathrm{~d}, J=8.0 \mathrm{~Hz}, 1 \mathrm{H}), 5.19(\mathrm{ABq}, J=14.4 \mathrm{~Hz}, 2 \mathrm{H}),{ }^{13} \mathrm{C} \mathrm{NMR}\left(\mathrm{CDCl}_{3}, 100 \mathrm{MHz}\right) \delta 140.8$, 139.1, 136.4, 131.9, 128.9, 128.5, 127.8, 127.7, 127.3, 126.7, 121.9, 121.0, 85.2, 72.8; HRMS calcd for $\mathrm{C}_{16} \mathrm{H}_{14} \mathrm{O}\left(\mathrm{M}^{+}\right) 222.1045$, found $222.1039\left(\mathrm{M}^{+}\right) ;[\alpha]^{16}{ }_{\mathrm{D}}=+4.7\left(c 1.1, \mathrm{CHCl}_{3}\right)$; ee $=88 \%$.

8g. ${ }^{1} \mathrm{H}$ NMR $\left(\mathrm{CDCl}_{3}, 400 \mathrm{MHz}\right) \delta 7.41(\mathrm{~d}, \mathrm{~J}=7.2 \mathrm{~Hz}, 2 \mathrm{H}), 7.32(\mathrm{t}, J=7.2 \mathrm{~Hz}, 2 \mathrm{H}), 7.19-7.12(\mathrm{~m}, 4 \mathrm{H})$, 7.04-7.01 (m, 1H), $6.71(\mathrm{~d}, J=15.6 \mathrm{~Hz}, 1 \mathrm{H}), 6.35$ (dd, $J=15.6,6.0 \mathrm{~Hz}, 1 \mathrm{H}), 4.95$ (bs, 2H), 4.41-4.36 $(\mathrm{m}, 1 \mathrm{H}), 2.93(\mathrm{dd}, J=16.0,10.8 \mathrm{~Hz}, 1 \mathrm{H}), 2.86(\mathrm{dd}, J=16.0,3.6 \mathrm{~Hz}, 1 \mathrm{H}) ;{ }^{13} \mathrm{C} \mathrm{NMR}\left(\mathrm{CDCl}_{3}, 100\right.$ MHz) $\delta 134.4,129.4,128.7,128.5,127.6,126.5,126.4,126.1,124.7,124.1,75.1,68.0$, 34.3; HRMS calcd for $\mathrm{C}_{17} \mathrm{H}_{16} \mathrm{O}\left(\mathrm{M}^{+}\right) 236.1201$, found $259.1093(\mathrm{M}+\mathrm{Na})$; $[\alpha]^{15}{ }_{\mathrm{D}}=-22.4\left(\right.$ c $\left.0.4, \mathrm{CHCl}_{3}\right)$; ee $=86 \%$.

8h. ${ }^{1} \mathrm{H}$ NMR $\left(\mathrm{CDCl}_{3}, 400 \mathrm{MHz}\right) \delta 7.57$ (d, $\left.J=8.4 \mathrm{~Hz}, 2 \mathrm{H}\right), 7.50$ (d, $\left.J=8.4 \mathrm{~Hz}, 2 \mathrm{H}\right), 7.25-7.02$ (m, 4H), $6.75(\mathrm{~d}, J=16.0 \mathrm{~Hz}, 1 \mathrm{H}), 6.44(\mathrm{dd}, J=16.0 \mathrm{~Hz}, 5.6 \mathrm{~Hz}, 1 \mathrm{H}), 4.94(\mathrm{ABq}, J=14.8 \mathrm{~Hz}, 2 \mathrm{H}), 4.43-$ $4.38(\mathrm{~m}, 1 \mathrm{H}), 3.00-2.84(\mathrm{~m}, 2 \mathrm{H}) ;{ }^{13} \mathrm{C} \mathrm{NMR}\left(\mathrm{CDCl}_{3}, 100 \mathrm{MHz}\right) \delta 140.1,134.2,132.6,132.0,129.6$, 
129.3, 128.7, 128.3, 126.6, 126.5, 126.2, 125.5, 125.4, 124.2, 74.7, 68.1, 34.2; HRMS calcd for $\mathrm{C}_{18} \mathrm{H}_{15} \mathrm{~F}_{3} \mathrm{O}\left(\mathrm{M}^{+}\right)$304.1075, found 304.1071 $\left(\mathrm{M}^{+}\right) ;[\alpha]^{25}=-13.2\left(\right.$ c 1.3, $\left.\mathrm{CHCl}_{3}\right)$; ee $=86 \%$.

8i. ${ }^{1} \mathrm{H}$ NMR $\left(\mathrm{CDCl}_{3}, 400 \mathrm{MHz}\right) \delta 7.35(\mathrm{~d}, J=8.4 \mathrm{~Hz}, 2 \mathrm{H}), 7.19-7.00(\mathrm{~m}, 4 \mathrm{H}), 6.85(\mathrm{~d}, J=8.4 \mathrm{~Hz}, 2 \mathrm{H})$, $6.65(\mathrm{~d}, J=15.6 \mathrm{~Hz}, 1 \mathrm{H}), 6.21(\mathrm{dd}, J=15.6,6.0 \mathrm{~Hz}, 1 \mathrm{H}), 4.93(\mathrm{ABq}, J=14.8 \mathrm{~Hz}, 2 \mathrm{H}), 4.39-4.32(\mathrm{~m}$, $1 \mathrm{H}), 3.81(\mathrm{~s}, 3 \mathrm{H}), 2.95(\mathrm{dd}, J=16.4,10.4 \mathrm{~Hz}, 1 \mathrm{H}), 2.85(\mathrm{dd}, J=16.4,3.6 \mathrm{~Hz}, 1 \mathrm{H}) ;{ }^{13} \mathrm{C} \mathrm{NMR}\left(\mathrm{CDCl}_{3}\right.$, $100 \mathrm{MHz}) \delta 159.2,134.5,132.9,130.6,129.4,128.8,127.7,127.2,126.4,126.0,124.1,113.9,75.3$, 68.0, 55.3, 34.4; HRMS calcd for $\mathrm{C}_{18} \mathrm{H}_{18} \mathrm{O}_{2}\left(\mathrm{M}^{+}\right)$266.1307, found 266.1302 (M $\left.{ }^{+}\right)$; $[\alpha]^{24}{ }_{\mathrm{D}}=-16.9$ (c 0.9, $\left.\mathrm{CHCl}_{3}\right)$; ee $=42 \%$.

Hydrogenation of 8a: To a solution of 8a (100 mg) in methanol $(5 \mathrm{~mL})$ was added $10 \mathrm{~mol} \% \mathrm{Pd} / \mathrm{C}(20$ $\mathrm{mg}$ ) and the mixture was stirred for $12 \mathrm{~h}$ at room temperature. The mixture was filtered through celite and washed with ethyl acetate. The combined filtrates were evaporated to give a residue which on column chromatography purification gave $12(\sim 70 \mathrm{mg}, 68 \%)$ in pure form.

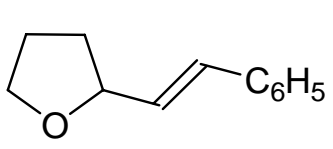

(S)-8a

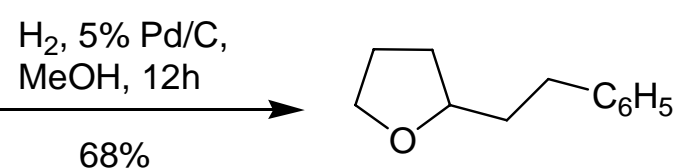

(S)-12

(S)-12. ${ }^{1} \mathrm{H} \mathrm{NMR}\left(\mathrm{CDCl}_{3}, 400 \mathrm{MHz}\right) \delta$ 7.29-7.15 (m, 5H), 3.91-3.78 (m, 2H), 3.73 (q, J = 8.0 Hz, 1H),

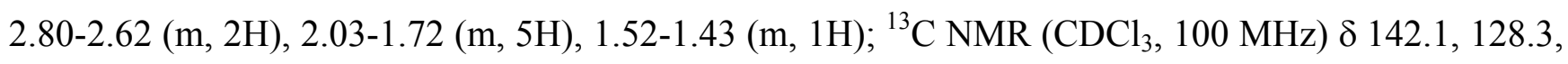
128.2, 125.6, 78.6, 67.7, 37.5, 32.7, 31.4, 25.8; HRMS calcd for $\mathrm{C}_{12} \mathrm{H}_{16} \mathrm{O}\left(\mathrm{M}^{+}\right)$176.1201, found $176.1196\left(\mathrm{M}^{+}\right) ;[\alpha]_{\mathrm{D}}{ }^{25}=+2.8\left(c 1.0, \mathrm{CHCl}_{3}\right)$. 


\section{Catalytic Asymmetric Hydrocarbonation of Alkynes}

The substrates $9 \mathbf{a}-\mathbf{h}$ were prepared by the previously reported procedure, see ref $10 \mathrm{a}$ in the text.

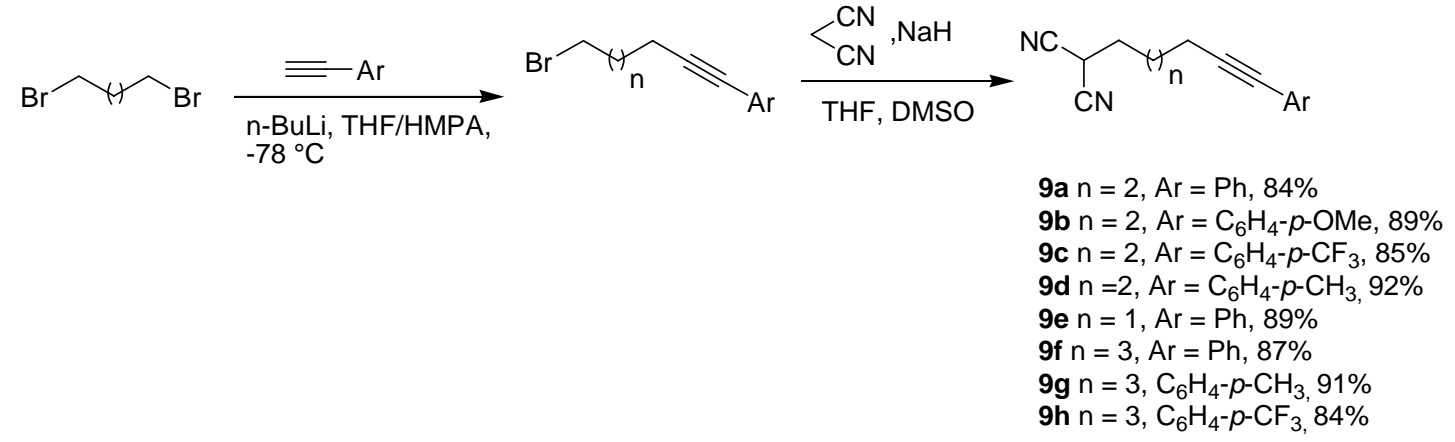

9a. ${ }^{1} \mathrm{H}$ NMR $\left(\mathrm{CDCl}_{3}, 400 \mathrm{MHz}\right) \delta$ 7.33-7.29 (m, 2H), 7.24-7.19 (m, 3H), $3.68(\mathrm{t}, J=6.8 \mathrm{~Hz}, 1 \mathrm{H}), 2.42$ $(\mathrm{t}, J=6.8 \mathrm{~Hz}, 2 \mathrm{H}), 2.04(\mathrm{q}, J=7.2 \mathrm{~Hz}, 2 \mathrm{H}), 1.80-1.72(\mathrm{~m}, 2 \mathrm{H}), 1.68-1.60(\mathrm{~m}, 2 \mathrm{H}) ;{ }^{13} \mathrm{C} \mathrm{NMR}\left(\mathrm{CDCl}_{3}\right.$, $100 \mathrm{MHz}) \delta 131.5,128.2,127.7,123.4,112.4,88.4,81.7,30.5,27.3,25.8,22.7,19.0$; HRMS calcd for $\mathrm{C}_{15} \mathrm{H}_{14} \mathrm{~N}_{2}\left(\mathrm{M}^{+}\right)$222.1157, found 245.1155 (M+Na).

9b. ${ }^{1} \mathrm{H}$ NMR $\left(\mathrm{CDCl}_{3}, 400 \mathrm{MHz}\right) \delta 7.32(\mathrm{~d}, J=9.2 \mathrm{~Hz}, 2 \mathrm{H}), 8.00(\mathrm{~d}, J=9.2 \mathrm{~Hz}, 2 \mathrm{H}), 3.79(\mathrm{~s}, 3 \mathrm{H}), 3.73$ $(\mathrm{t}, J=6.8 \mathrm{~Hz}, 1 \mathrm{H}), 2.46(\mathrm{t}, J=6.8 \mathrm{~Hz}, 2 \mathrm{H}), 2.08(\mathrm{q}, J=7.2 \mathrm{~Hz}, 2 \mathrm{H}), 1.85-1.77(\mathrm{~m}, 2 \mathrm{H}), 1.72-1.65(\mathrm{~m}$, $2 \mathrm{H}) ;{ }^{13} \mathrm{C} \mathrm{NMR}\left(\mathrm{CDCl}_{3}, 100 \mathrm{MHz}\right) \delta 159.1,132.8,115.6,113.8,112.4,86.9,81.4,55.2,30.4,27.4$, 25.8, 22.6, 18.9; HRMS calcd for $\mathrm{C}_{16} \mathrm{H}_{16} \mathrm{~N}_{2} \mathrm{O}\left(\mathrm{M}^{+}\right)$252.1263, found $275.1155(\mathrm{M}+\mathrm{Na})$.

9c. ${ }^{1} \mathrm{H} \mathrm{NMR}\left(\mathrm{CDCl}_{3}, 400 \mathrm{MHz}\right) \delta 7.53(\mathrm{~d}, J=8.0 \mathrm{~Hz}, 2 \mathrm{H}), 7.48(\mathrm{~d}, J=8.0 \mathrm{~Hz}, 2 \mathrm{H}), 3.76(\mathrm{t}, J=6.8 \mathrm{~Hz}$, $1 \mathrm{H}), 2.50(\mathrm{t}, J=6.8 \mathrm{~Hz}, 2 \mathrm{H}), 2.00(\mathrm{q}, J=7.2 \mathrm{~Hz}, 2 \mathrm{H}), 1.86-1.77(\mathrm{~m}, 2 \mathrm{H}), 1.74-1.65(\mathrm{~m}, 2 \mathrm{H}) ;{ }^{13} \mathrm{C} \mathrm{NMR}$ $\left(\mathrm{CDCl}_{3}, 100 \mathrm{MHz}\right) \delta 131.7,125.1,125.1,125.0,124.9,112.4,91.3,91.2,80.4,30.3,27.1,25.7,22.6$, 18.9; HRMS calcd for $\mathrm{C}_{16} \mathrm{H}_{13} \mathrm{~F}_{3} \mathrm{~N}_{2}\left(\mathrm{M}^{+}\right)$290.1031, found $313.0923(\mathrm{M}+\mathrm{Na})$.

9d. ${ }^{1} \mathrm{H}$ NMR $\left(\mathrm{CDCl}_{3}, 400 \mathrm{MHz}\right) \delta 7.27(\mathrm{dd}, J=8.0,1.2 \mathrm{~Hz}, 2 \mathrm{H}), 7.08(\mathrm{~d}, J=8.0 \mathrm{~Hz}, 2 \mathrm{H}), 3.70(\mathrm{t}, J=$ $7.2 \mathrm{~Hz}, 1 \mathrm{H}), 2.46$ (t, $J=6.8 \mathrm{~Hz}, 2 \mathrm{H}), 2.32$ (s, 3H), 2.06 (q, $J=6.8 \mathrm{~Hz}, 2 \mathrm{H}), 1.83-1.75$ (m, 2H), 1.71$1.63(\mathrm{~m}, 2 \mathrm{H}) ;{ }^{13} \mathrm{C} \mathrm{NMR}\left(\mathrm{CDCl}_{3}, 100 \mathrm{MHz}\right) \delta 137.7,131.3,128.9,120.3,112.4,87.7,81.6,30.4,27.3$, 25.7, 22.6, 21.4, 18.9; HRMS calcd for $\mathrm{C}_{16} \mathrm{H}_{16} \mathrm{~N}_{2}\left(\mathrm{M}^{+}\right)$236.1313, found 359.1206 (M+Na). 
9e. ${ }^{1} \mathrm{H}$ NMR $\left(\mathrm{CDCl}_{3}, 400 \mathrm{MHz}\right) \delta$ 7.33-7.29 (m, 2H), 7.22-7.18 (m, 3H), 3.69 (t, J=6.8 Hz, 1H), 2.42 $(\mathrm{t}, J=6.8 \mathrm{~Hz}, 2 \mathrm{H}), 2.08(\mathrm{q}, J=6.8 \mathrm{~Hz}, 2 \mathrm{H}), 1.81-1.73(\mathrm{~m}, 2 \mathrm{H}) ;{ }^{13} \mathrm{C} \mathrm{NMR}\left(\mathrm{CDCl}_{3}, 100 \mathrm{MHz}\right) \delta 131.3$, 128.1, 127.9, 122.9, 112.4, 87.2, 82.2, 29.8, 25.2, 22.2, 18.2; HRMS calcd for $\mathrm{C}_{14} \mathrm{H}_{12} \mathrm{~N}_{2}\left(\mathrm{M}^{+}\right)$ 208.1001, found $231.0893(\mathrm{M}+\mathrm{Na})$.

9f. ${ }^{1} \mathrm{H}$ NMR $\left(\mathrm{CDCl}_{3}, 400 \mathrm{MHz}\right) \delta$ 7.37-7.34 (m, 2H), 7.26-7.21 (m, 3H), $3.65(\mathrm{t}, J=6.8 \mathrm{~Hz}, 1 \mathrm{H}), 2.40$ $(\mathrm{t}, J=6.8 \mathrm{~Hz}, 2 \mathrm{H}), 2.02-1.96(\mathrm{~m}, 2 \mathrm{H}), 1.63-1.40(\mathrm{~m}, 6 \mathrm{H}) ;{ }^{13} \mathrm{C} \mathrm{NMR}\left(\mathrm{CDCl}_{3}, 100 \mathrm{MHz}\right) \delta 131.4,128.1$, 127.5, 123.6, 112.5, 89.3, 81.0, 30.6, 28.0, 27.5, 26.1, 22.5, 19.1; HRMS calcd for $\mathrm{C}_{16} \mathrm{H}_{16} \mathrm{~N}_{2}\left(\mathrm{M}^{+}\right)$ 236.1314, found $259.1312(\mathrm{M}+\mathrm{Na})$.

9g. ${ }^{1} \mathrm{H}$ NMR $\left(\mathrm{CDCl}_{3}, 400 \mathrm{MHz}\right) \delta 7.27(\mathrm{~d}, J=8.0 \mathrm{~Hz}, 2 \mathrm{H}), 7.08(\mathrm{~d}, J=8.0 \mathrm{~Hz}, 2 \mathrm{H}), 3.69(\mathrm{t}, J=6.8$ $\mathrm{Hz}, 1 \mathrm{H}), 2.43(\mathrm{t}, J=6.8 \mathrm{~Hz}, 2 \mathrm{H}), 2.33(\mathrm{~s}, 3 \mathrm{H}), 2.03(\mathrm{q}, J=6.8 \mathrm{~Hz}, 2 \mathrm{H}), 1.70-1.51(\mathrm{~m}, 6 \mathrm{H}) ;{ }^{13} \mathrm{C} \mathrm{NMR}$ $\left(\mathrm{CDCl}_{3}, 100 \mathrm{MHz}\right) \delta 137.6,131.3,128.9,120.6,112.5,88.5,81.1,30.7,28.1,27.5,26.2,22.6,21.4$, 19.2; HRMS calcd for $\mathrm{C}_{17} \mathrm{H}_{18} \mathrm{~N}_{2}\left(\mathrm{M}^{+}\right)$250.1470, found $273.1362(\mathrm{M}+\mathrm{Na})$.

9h. ${ }^{1} \mathrm{H} \mathrm{NMR}\left(\mathrm{CDCl}_{3}, 400 \mathrm{MHz}\right) \delta 7.51(\mathrm{~d}, J=8.4 \mathrm{~Hz}, 2 \mathrm{H}), 7.45(\mathrm{~d}, J=8.4 \mathrm{~Hz}, 2 \mathrm{H}), 3.73(\mathrm{t}, J=6.8$ $\mathrm{Hz}, 1 \mathrm{H}), 2.43(\mathrm{t}, J=6.8 \mathrm{~Hz}, 2 \mathrm{H}), 2.01(\mathrm{q}, J=6.8 \mathrm{~Hz}, 2 \mathrm{H}), 1.68-1.40(\mathrm{~m}, 6 \mathrm{H}) ;{ }^{13} \mathrm{C} \mathrm{NMR}\left(\mathrm{CDCl}_{3}, 100\right.$ MHz) $\delta 131.6,127.5,125.0,112.5,92.2,79.9,30.6,27.9,27.5,26.1,22.6,19.1$; HRMS calcd for $\mathrm{C}_{17} \mathrm{H}_{15} \mathrm{~F}_{3} \mathrm{~N}_{2}\left(\mathrm{M}^{+}\right)$304.1187, found $327.1080(\mathrm{M}+\mathrm{Na})$.

\section{Catalytic Asymmetric Cyclization of Alkynylmethines 9a-h:}

The alkynylmethines 9a-h were subjected to palladium catalysis under condition $\mathbf{A}$ or $\mathbf{B}$ to give corresponding carbocycles 10a-h. Refer Table 4 in the text.

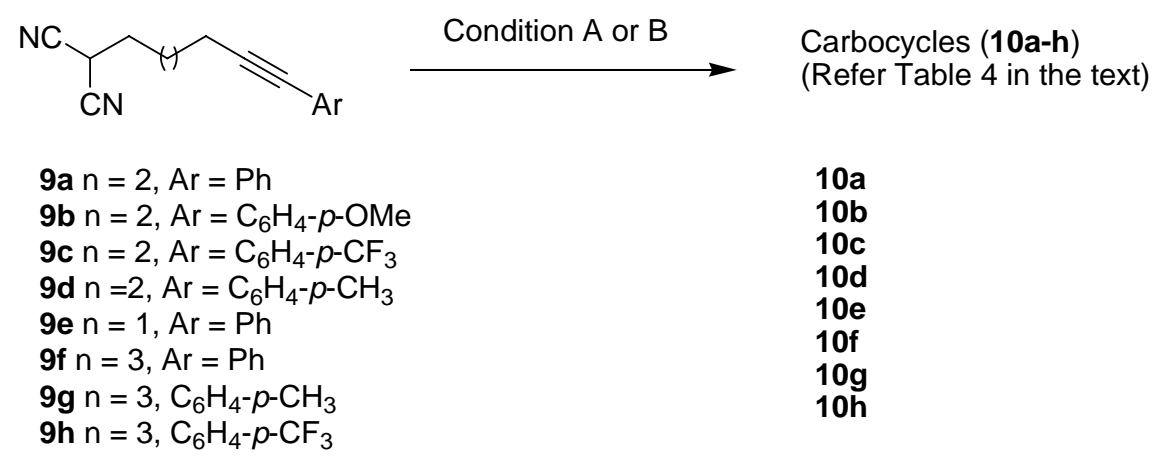


10a. ${ }^{1} \mathrm{H} \mathrm{NMR}\left(\mathrm{CDCl}_{3}, 400 \mathrm{MHz}\right) \delta 7.36-7.17(\mathrm{~m}, 5 \mathrm{H}), 6.63(\mathrm{~d}, J=16 \mathrm{~Hz}, 1 \mathrm{H}), 6.13(\mathrm{dd}, J=16.0,8.4$ $\mathrm{Hz}, 1 \mathrm{H}), 3.06$ (q, $J=8.4 \mathrm{~Hz}, 1 \mathrm{H}), 2.54(\mathrm{ddd}, \mathrm{J}=13.2,8.4,4.8 \mathrm{~Hz}, 1 \mathrm{H}), 2.37-2.29$ (m, 1H), 2.14-1.82 $(\mathrm{m}, 4 \mathrm{H}) ;{ }^{13} \mathrm{C} \mathrm{NMR}\left(\mathrm{CDCl}_{3}, 100 \mathrm{MHz}\right) \delta 135.6,135.5,128.6,128.3,126.6,123.6,115.9,114.4,54.6$, 40.7, 38.3, 30.0, 22.3; HRMS calcd for $\mathrm{C}_{15} \mathrm{H}_{14} \mathrm{~N}_{2}\left(\mathrm{M}^{+}\right)$222.1157, found 245.1049 $(\mathrm{M}+\mathrm{Na})$; $[\alpha]^{25}=-$ $155.6\left(c 0.4, \mathrm{CHCl}_{3}\right)$; ee $=90 \%$

10b. ${ }^{1} \mathrm{H}$ NMR $\left(\mathrm{CDCl}_{3}, 400 \mathrm{MHz}\right) \delta 7.35(\mathrm{~d}, J=8.8 \mathrm{~Hz}, 2 \mathrm{H}), 6.85(\mathrm{~d}, J=8.8 \mathrm{~Hz}, 2 \mathrm{H}), 6.64(\mathrm{~d}, J=16.0$ $\mathrm{Hz}, 1 \mathrm{H}), 6.06(\mathrm{dd}, J=16.0,8.8 \mathrm{~Hz}, 1 \mathrm{H}), 3.81(\mathrm{~s}, 3 \mathrm{H}), 3.12(\mathrm{q}, J=8.8 \mathrm{~Hz}, 1 \mathrm{H}), 2.60$ (ddd, $J=13.2,8.8$, $4.8 \mathrm{~Hz}, 1 \mathrm{H}), 2.44-2.37(\mathrm{~m}, 1 \mathrm{H}), 2.21-1.89(\mathrm{~m}, 4 \mathrm{H}) ;{ }^{13} \mathrm{C} \mathrm{NMR}\left(\mathrm{CDCl}_{3}, 100 \mathrm{MHz}\right) \delta 159.7,134.9,128.5$, 127.9, 121.3, 115.9, 114.5, 114.0, 55.3, 54.8, 40.8, 38.3, 30.1, 22.4; HRMS calcd for $\mathrm{C}_{16} \mathrm{H}_{16} \mathrm{~N}_{2} \mathrm{O}\left(\mathrm{M}^{+}\right)$ 252.1263 , found $275.1155(\mathrm{M}+\mathrm{Na}) ;[\alpha]^{25}=+46.9\left(c 0.4, \mathrm{CHCl}_{3}\right)$; ee $=71 \%$

10c. ${ }^{1} \mathrm{H}$ NMR $\left(\mathrm{CDCl}_{3}, 400 \mathrm{MHz}\right) \delta 7.58(\mathrm{~d}, J=8.0 \mathrm{~Hz}, 2 \mathrm{H}), 7.50(\mathrm{~d}, J=8.0 \mathrm{~Hz}, 2 \mathrm{H}), 6.74(\mathrm{~d}, J=16.0$ $\mathrm{Hz}, 1 \mathrm{H}), 6.31(\mathrm{dd}, J=16.0,8.4 \mathrm{~Hz}, 1 \mathrm{H}), 3.17$ (q, $J=8.8 \mathrm{~Hz}, 1 \mathrm{H}), 2.63(\mathrm{ddd}, J=13.2,8.4,4.8 \mathrm{~Hz}, 1 \mathrm{H})$,

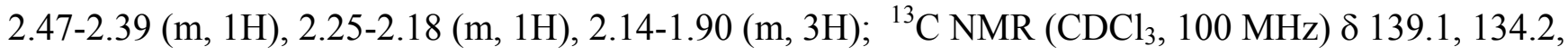
126.5, 125.6, 125.5, 115.7, 114.2, 54.4, 40.6, 38.3, 29.9, 22.4; HRMS calcd for $\mathrm{C}_{16} \mathrm{H}_{13} \mathrm{~F}_{3} \mathrm{~N}_{2}\left(\mathrm{M}^{+}\right)$ 290.1031 , found $313.0923(\mathrm{M}+\mathrm{Na}) ;[\alpha]^{25}=-31.3\left(c 0.3, \mathrm{CHCl}_{3}\right)$; ee $=68 \%$

10d. ${ }^{1} \mathrm{H}$ NMR $\left(\mathrm{CDCl}_{3}, 400 \mathrm{MHz}\right) \delta 7.30(\mathrm{~d}, J=8.0 \mathrm{~Hz}, 2 \mathrm{H}), 7.13(\mathrm{~d}, J=8.0 \mathrm{~Hz}, 2 \mathrm{H}), 6.66(\mathrm{~d}, J=15.6$ $\mathrm{Hz}, 1 \mathrm{H}), 6.15(\mathrm{dd}, J=15.6,8.4 \mathrm{~Hz}, 1 \mathrm{H}), 3.14$ (q, $J=8.0 \mathrm{~Hz}, 1 \mathrm{H}), 2.60(\mathrm{ddd}, J=13.2,8.4,4.8 \mathrm{~Hz}, 1 \mathrm{H})$, 2.44-2.38 (m, 1H), 3.34 (s, 3H), 2.21-1.87 (m, 4H); ${ }^{13} \mathrm{C} \mathrm{NMR}\left(\mathrm{CDCl}_{3}, 100 \mathrm{MHz}\right) \delta 138.2,135.4,132.9$, 129.2, 126.5, 122.6, 115.9, 114.4, 54.7, 40.7, 38.3, 30.1, 22.3, 21.3; HRMS calcd for $\mathrm{C}_{16} \mathrm{H}_{16} \mathrm{~N}_{2}\left(\mathrm{M}^{+}\right)$ 236.1313 , found $259.1206(\mathrm{M}+\mathrm{Na}) ;[\alpha]^{25} \mathrm{D}=+25.4\left(c 0.35, \mathrm{CHCl}_{3}\right)$; ee $=88 \%$

10e. ${ }^{1} \mathrm{H} \mathrm{NMR}\left(\mathrm{CDCl}_{3}, 400 \mathrm{MHz}\right) \delta 7.40(\mathrm{~s}, 5 \mathrm{H}), 6.07-6.04(\mathrm{~m}, 1 \mathrm{H}), 5.72(\mathrm{ddd}, J=6.8,4.8,2.4 \mathrm{~Hz}$, $1 \mathrm{H}), 3.93(\mathrm{t}, J=2.4 \mathrm{~Hz}, 1 \mathrm{H}), 2.53-2.29(\mathrm{~m}, 4 \mathrm{H}) ;{ }^{13} \mathrm{C} \mathrm{NMR}\left(\mathrm{CDCl}_{3}, 100 \mathrm{MHz}\right) \delta 136.1,129.2,128.9$, 128.7, 128.0, 124.9, 115.7, 113.7, 48.0, 38.0, 30.5, 21.5; HRMS calcd for $\mathrm{C}_{14} \mathrm{H}_{12} \mathrm{~N}_{2}\left(\mathrm{M}^{+}\right)$208.1001, found $231.1001(\mathrm{M}+\mathrm{Na}) ;[\alpha]_{\mathrm{D}}^{25}=-29.8\left(c 0.32, \mathrm{CHCl}_{3}\right)$; determination of ee proved difficult.

10f. ${ }^{1} \mathrm{H} \mathrm{NMR}\left(\mathrm{CDCl}_{3}, 400 \mathrm{MHz}\right) \delta 7.35-7.16(\mathrm{~m}, 5 \mathrm{H}), 6.60(\mathrm{~d}, J=15.6 \mathrm{~Hz}, 1 \mathrm{H}), 6.10(\mathrm{dd}, J=15.6,8.8$ $\mathrm{Hz}, 1 \mathrm{H}), 2.53(\mathrm{ddd}, J=11.6,8.8,7.2 \mathrm{~Hz}, 1 \mathrm{H}), 2.40$ (d, $J=13.2 \mathrm{~Hz}, 1 \mathrm{H}), 1.93-1.76(\mathrm{~m}, 4 \mathrm{H}), 1.65-1.52$ 
(m, 2H), 1.35-1.28 (m, 1H); ${ }^{13} \mathrm{C} \mathrm{NMR}\left(\mathrm{CDCl}_{3}, 100 \mathrm{MHz}\right) \delta 135.8,134.9,128.5,128.1,126.6,125.8$, 115.9, 113.9, 47.7, 39.0, 35.1, 28.1, 23.9, 21.3; HRMS calcd for $\mathrm{C}_{16} \mathrm{H}_{16} \mathrm{~N}_{2}\left(\mathrm{M}^{+}\right)$236.1313, found $259.1206(\mathrm{M}+\mathrm{Na}) ;[\alpha]^{25}{ }_{\mathrm{D}}=-175.4\left(c 0.33, \mathrm{CHCl}_{3}\right)$; ee $=72 \%$

10g. ${ }^{1} \mathrm{H}$ NMR $\left(\mathrm{CDCl}_{3}, 400 \mathrm{MHz}\right) \delta 7.30(\mathrm{~d}, J=8.0 \mathrm{~Hz}, 2 \mathrm{H}), 7.13(\mathrm{~d}, J=8.0 \mathrm{~Hz}, 2 \mathrm{H}), 6.63(\mathrm{~d}, J=15.6$ Hz, 1H), 6.12 (dd, $J=15.6,8.4 \mathrm{~Hz}, 1 \mathrm{H}), 2.58$ (dt, $J=8.4,3.2 \mathrm{~Hz}, 1 \mathrm{H}), 2.47$ (d, $J=14.0 \mathrm{~Hz}, 1 \mathrm{H}), 2.18$ (s, 3H), 1.99-1.85 (m, 4H), 1.70-1.57 (m, 2H), 1.42-1.32 (m, 1H); ${ }^{13} \mathrm{C}$ NMR $\left(\mathrm{CDCl}_{3}, 100 \mathrm{MHz}\right)$ $\delta$ 138.1, 134.7, 133.0, 129.2, 126.5, 124.7, 115.9, 113.9, 47.7, 39.1, 35.1, 28.2, 23.9, 21.3, 21.2; HRMS calcd for $\mathrm{C}_{17} \mathrm{H}_{18} \mathrm{~N}_{2}\left(\mathrm{M}^{+}\right) 250.1470$, found $273.1362(\mathrm{M}+\mathrm{Na}) ;[\alpha]^{25}=+19.3\left(\right.$ c $\left.0.53, \mathrm{CHCl}_{3}\right)$; ee $=76 \%$

10h. ${ }^{1} \mathrm{H}$ NMR $\left(\mathrm{CDCl}_{3}, 400 \mathrm{MHz}\right) \delta 7.58(\mathrm{~d}, J=8.4 \mathrm{~Hz}, 2 \mathrm{H}), 7.50(\mathrm{~d}, J=8.4 \mathrm{~Hz}, 2 \mathrm{H}), 7.00(\mathrm{~d}, J=15.6$ $\mathrm{Hz}, 1 \mathrm{H}), 2.28(\mathrm{dd}, J=15.6,8.8 \mathrm{~Hz}, 1 \mathrm{H}), 2.64(\mathrm{ddd}, J=11.2,8.8,2.8 \mathrm{~Hz}, 1 \mathrm{H}), 2.50(\mathrm{~d}, J=13.6 \mathrm{~Hz}$,

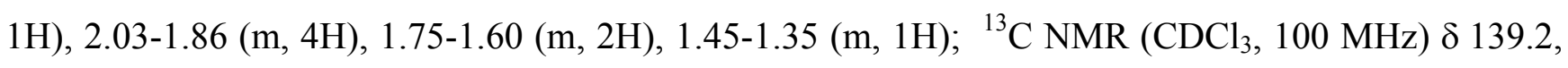
133.6, 128.5, 126.8, 125.6, 125.5, 125.5, 125.4, 125.3, 115.8, 113.8, 47.7, 38.8, 35.0, 27.9, 23.8, 21.2; HRMS calcd for $\mathrm{C}_{17} \mathrm{H}_{15} \mathrm{~F}_{3} \mathrm{~N}_{2}\left(\mathrm{M}^{+}\right)$304.1187, found $327.1080(\mathrm{M}+\mathrm{Na}) ;[\alpha]^{25}=+21.3\left(\right.$ c $\left.0.53, \mathrm{CHCl}_{3}\right)$; ee $=61 \%$ 

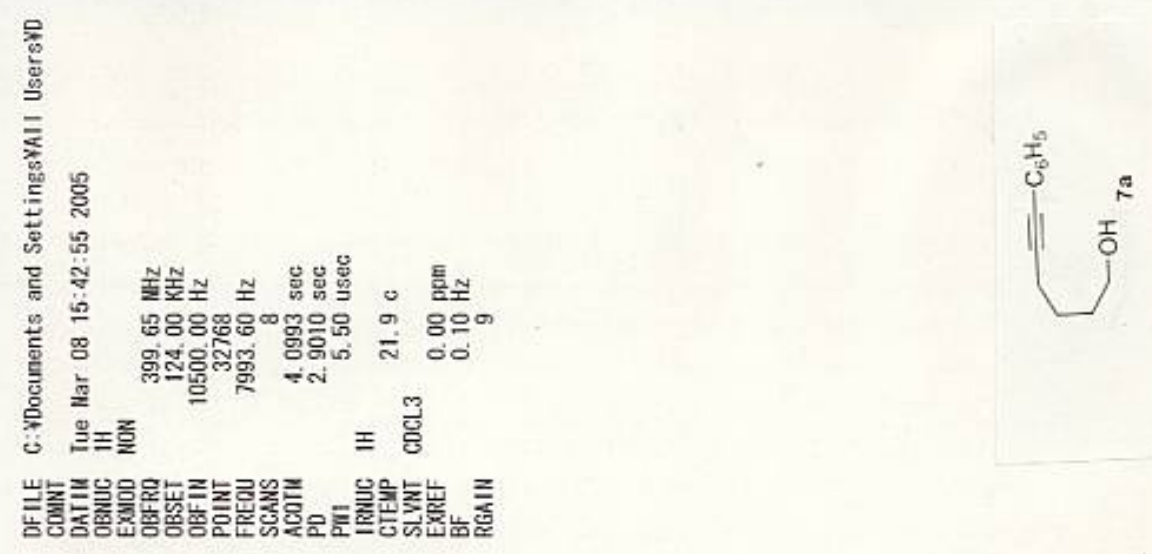

0000

$899^{\circ} 1-$
$\forall 99^{\circ} 1$

$799^{\circ} 1$
$0<9$

L89 $1-$

969 1-

$669 \%$

ILL $1-1$

IEL I-

LEL $1-1$

$\operatorname{tol} 1-$

IIL $1-$

$62+2-$

$900-2$
$29+\frac{2}{2}$

$819^{\circ} \mathrm{C}$

tol

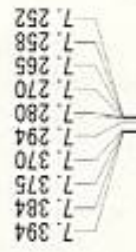

$00 \%$
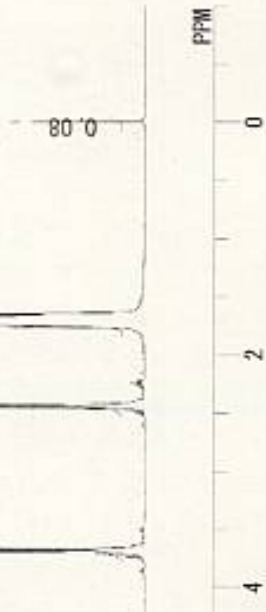

$-\infty$ 

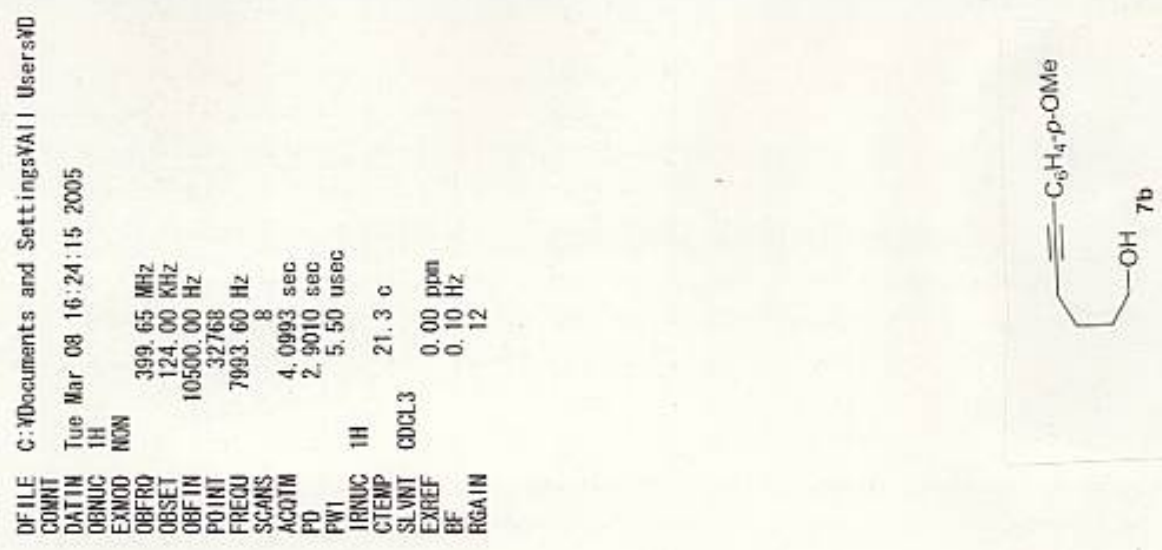

$0000^{\circ}$

$1 / 9^{\circ}$ I-

969 '

$92 L$.

$\varepsilon \rightarrow L$

6102

¿st?

989.8

101 '

$68 \mathrm{~L}$ ह

$86<$. $9-$

718.9

$908-$

goc 2 

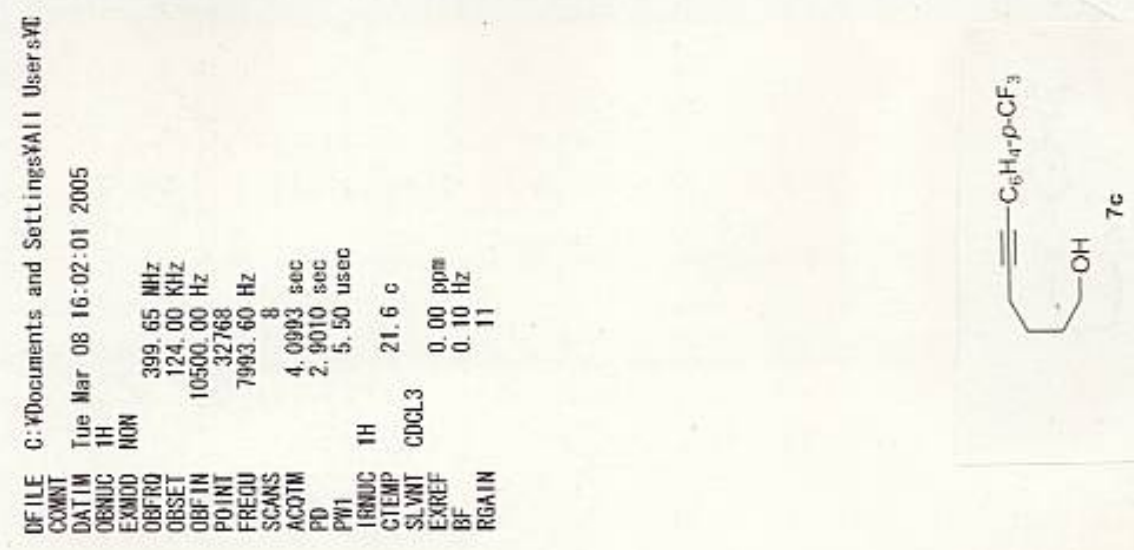

$0000^{\circ}$

$969.1-$

LOL:

III $1-$

$62 x-1=$

$\angle D L:$

$\operatorname{LgL}:-$

L9D 2

$\begin{array}{lll}2 L 7 \\ 06 & 2\end{array}$

$969^{\circ} 8$

2!L \&

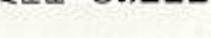

(2)

$652: 2$
$095 \cdot 2$
0952
$999 \cdot ?$
LES L

LES. 

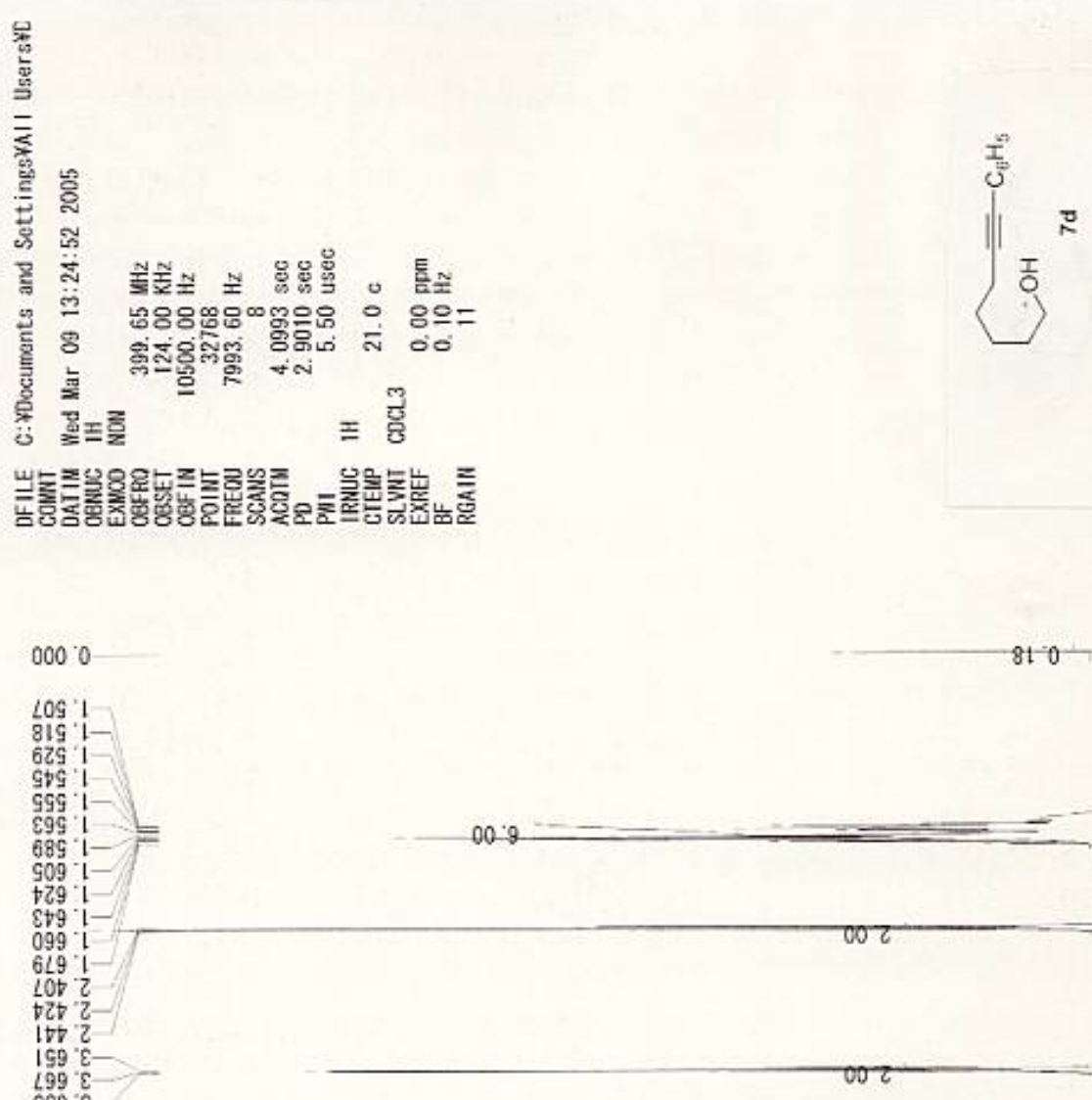

L99 $\mathrm{E}-$

$889^{\circ}$ है
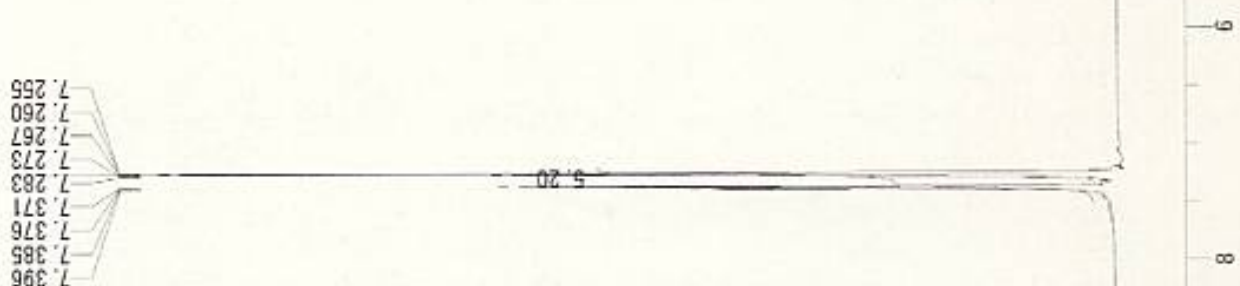

$\infty$ 

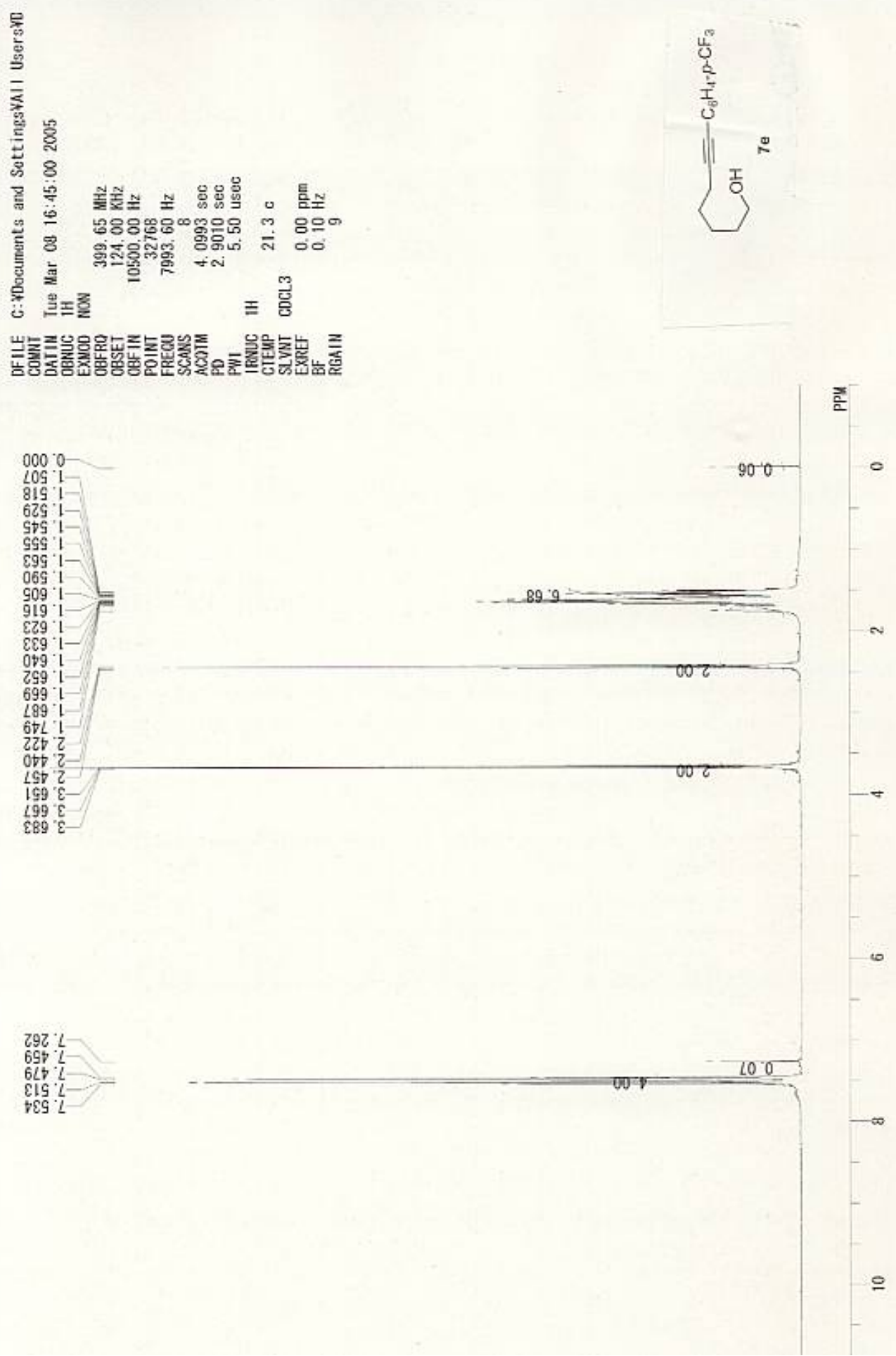

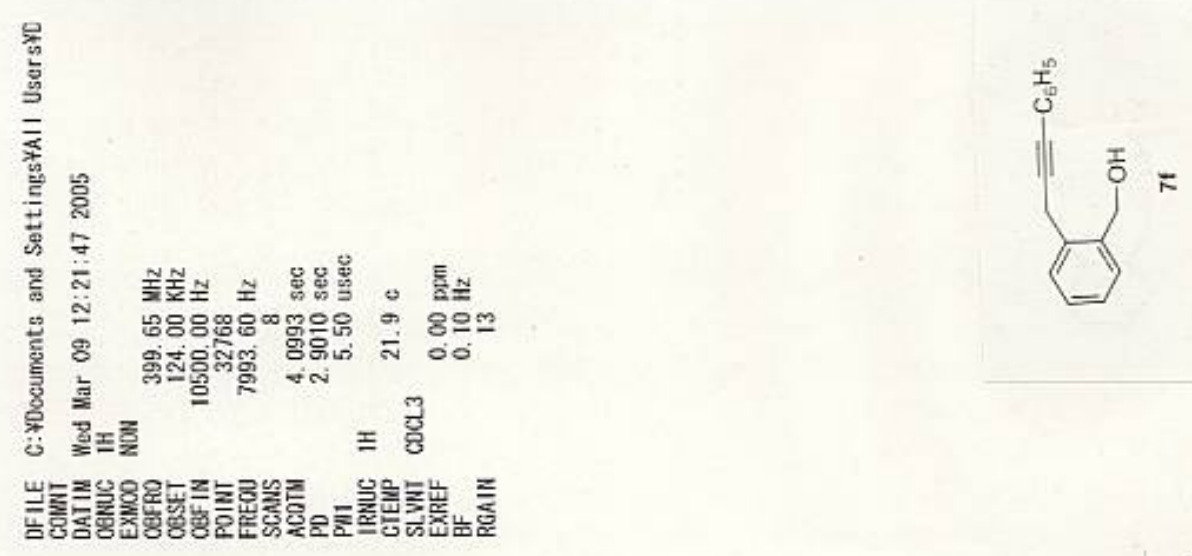

$000 \%$

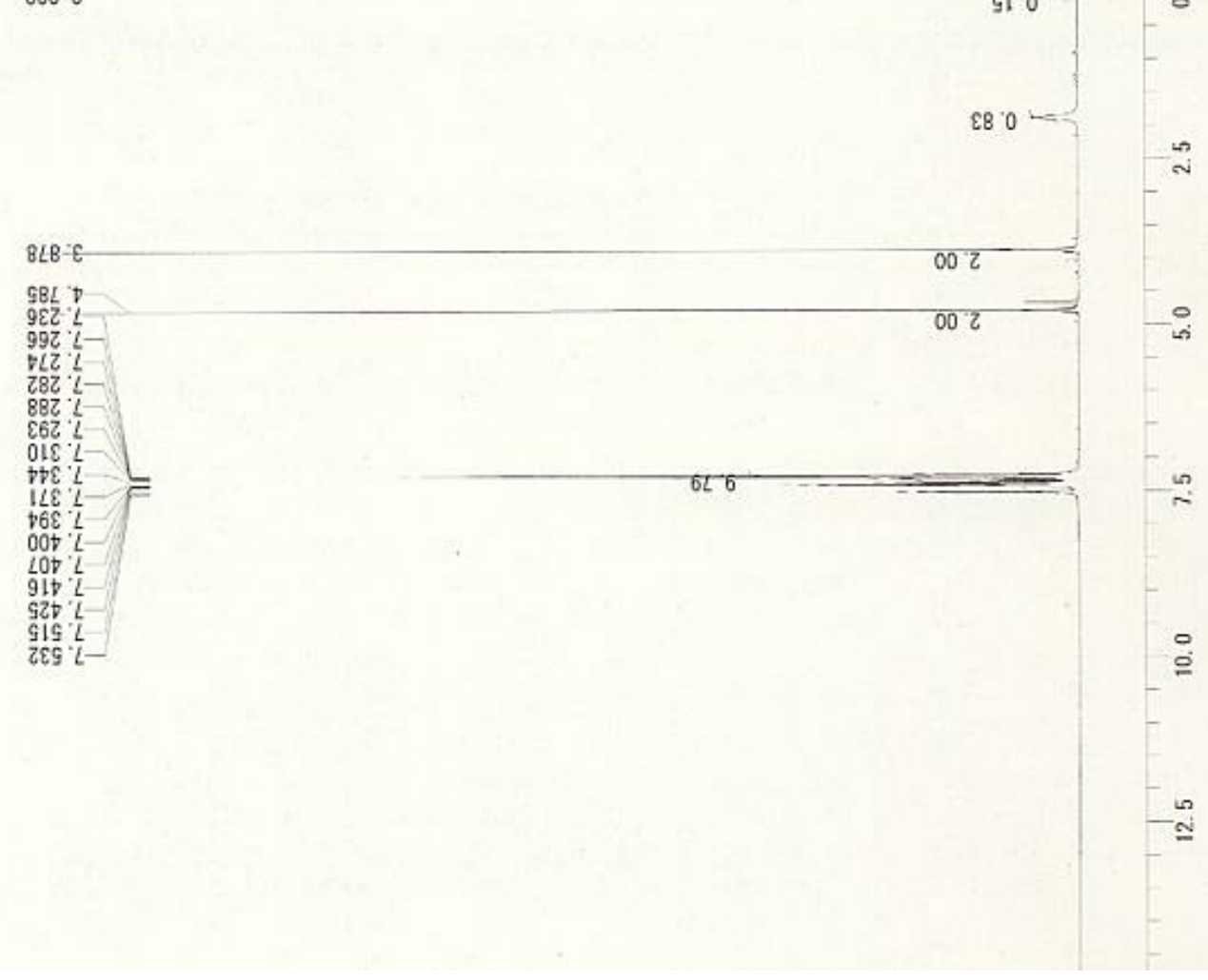



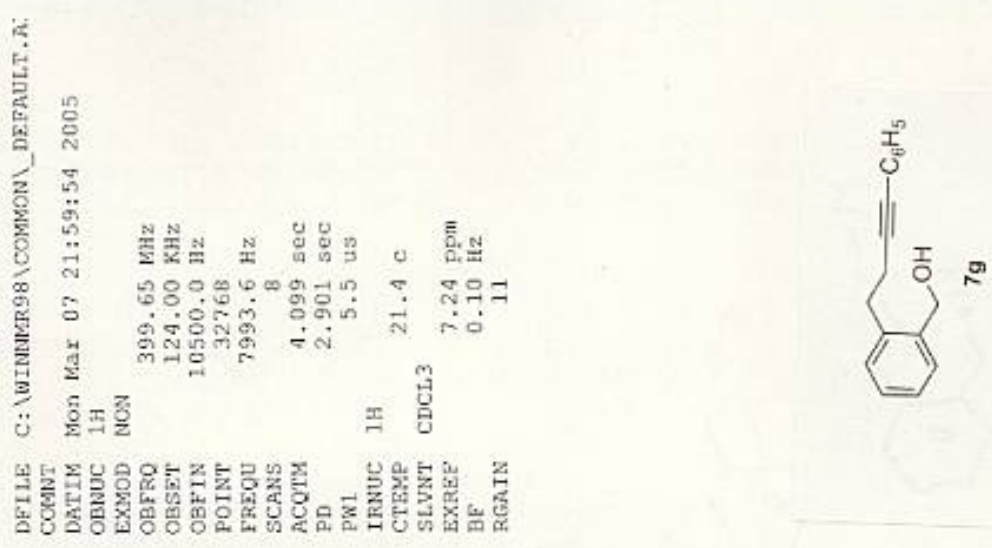

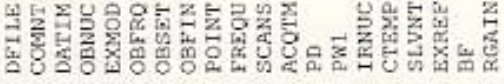



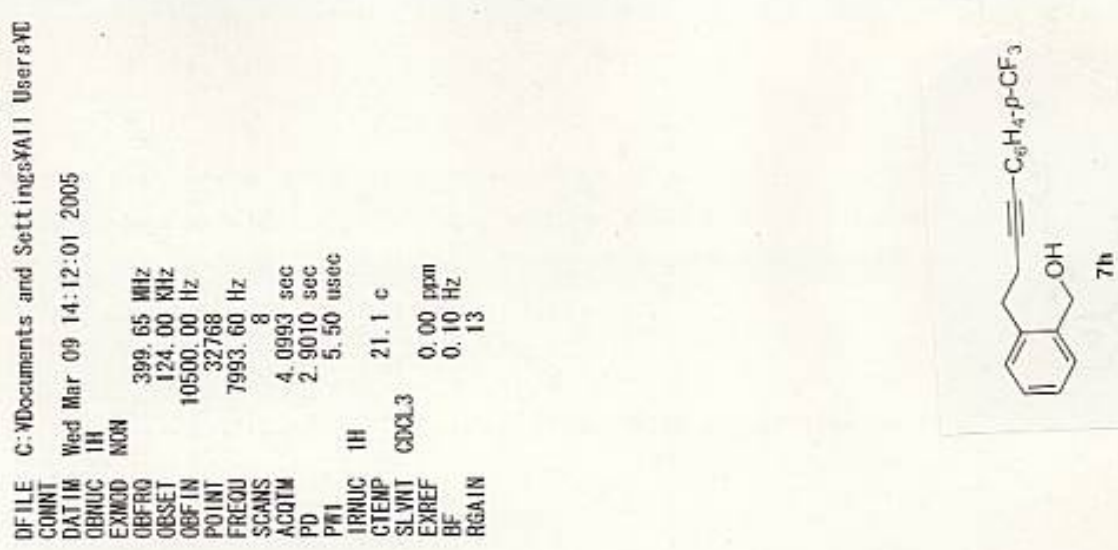

$000 \%$

$989^{\circ} 1-$

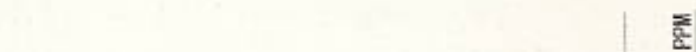

DEL ?-

\&gl ?

?LL?

710 -

$190^{\circ} \mathrm{E}$

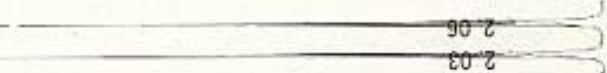

$99 L+7$

00

$\angle \nabla C 2$

212

$262 \cdot L$

$20 E^{2} L$

$69 \varepsilon^{2}=$

gटD ?

9652

609.2

629. 

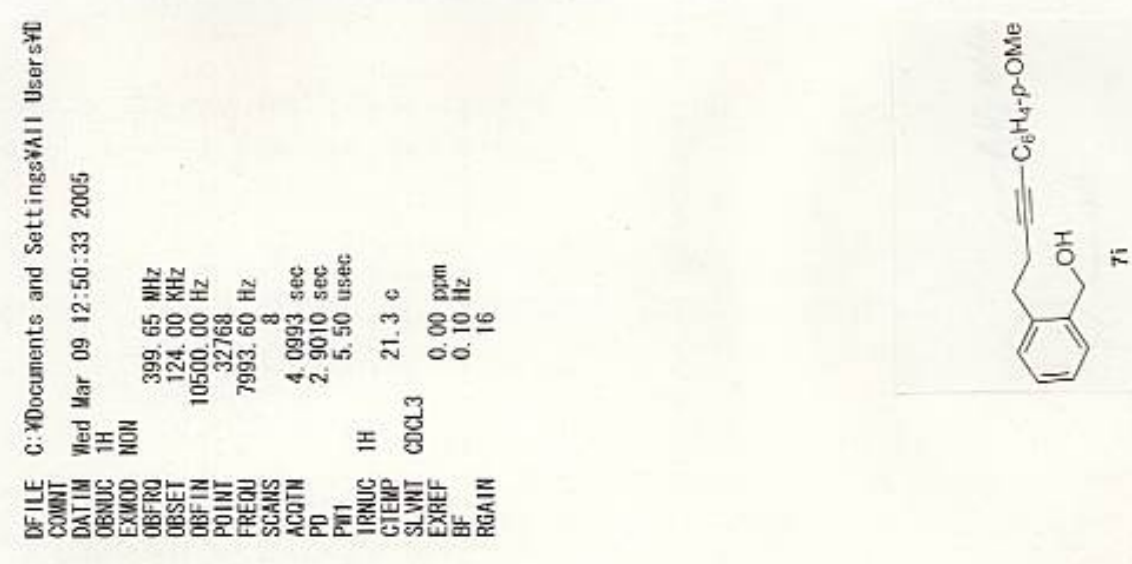

$000 \%$

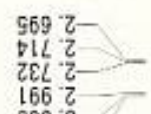

1662

$820^{\circ} 8$

$88 L-E$

$Z L L D$
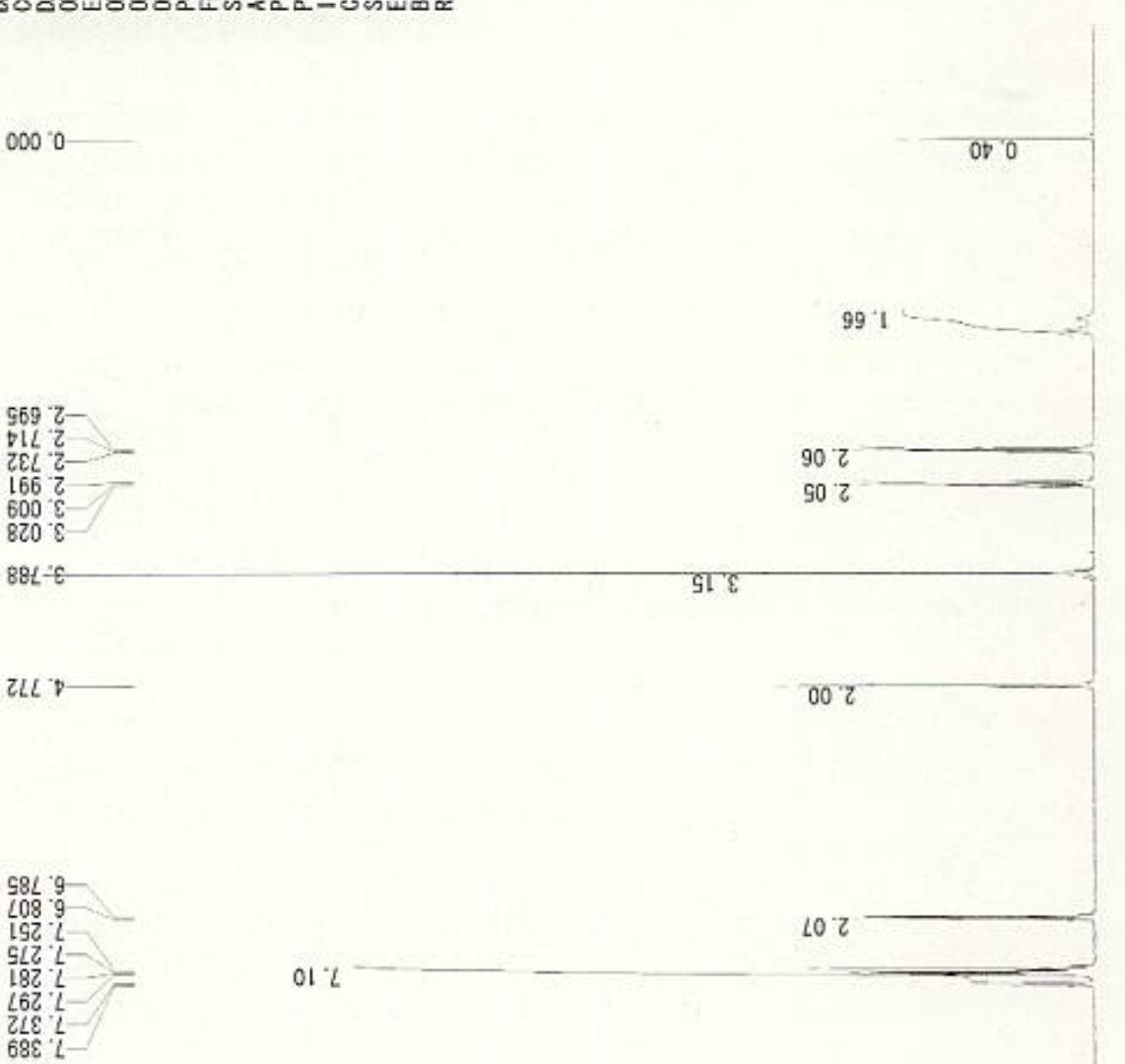

90

so 7

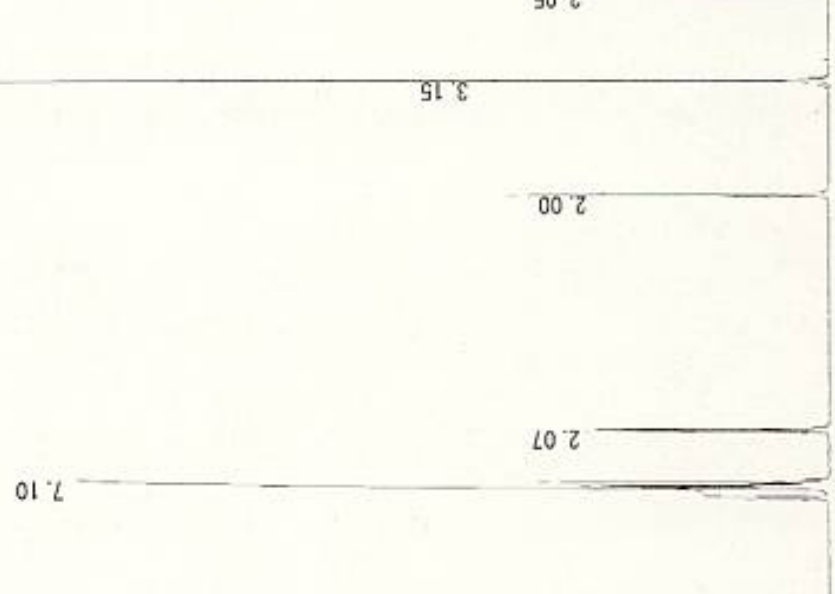

$-\infty$ 

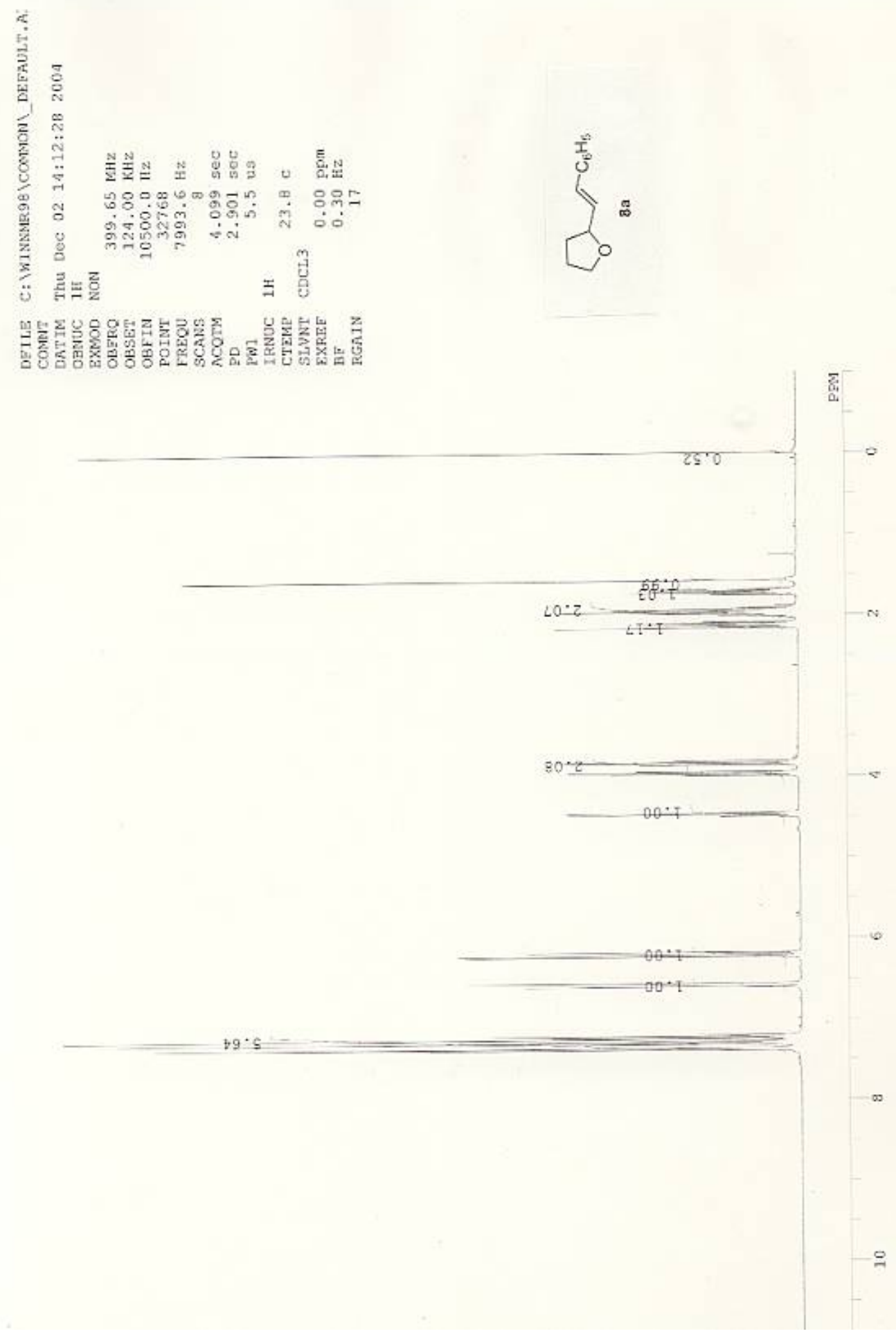

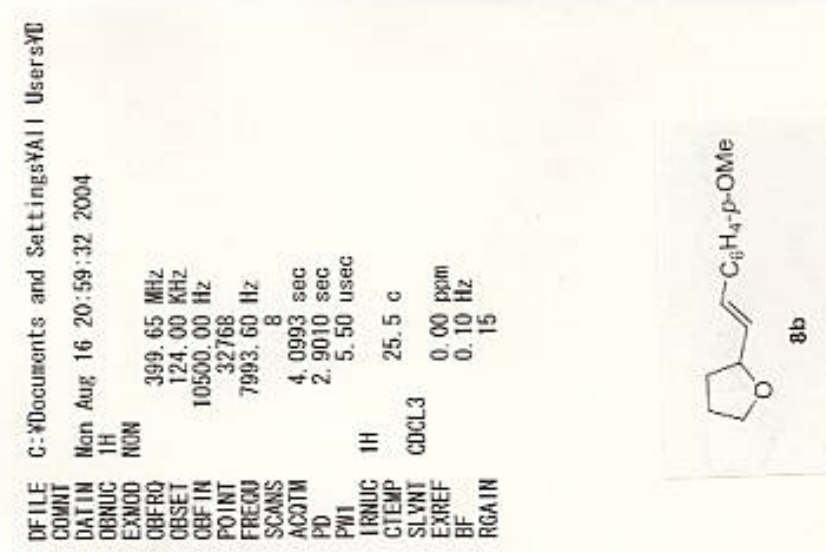

$000^{\circ}$
192.1

129.

289.1

2691

$22 L 1$

ive $1-$

$1260^{\circ}$

976 i

$296.1-$

$596^{\circ} 1-$
$2261-$

$186^{\circ}$

$786.1-$

$\angle 86^{\prime} 1$

$68611-$

480.2

280.2

$\angle 60 \%$

$9117-6$

191 ?

$261^{\circ} \mathrm{C}-$

608 ह

$718-8$

ECB 8

678 -

626 '

$\operatorname{Lt} 0^{\circ} \mathcal{E}$

$\mathrm{C} 96{ }^{\circ} \mathrm{E}$
$\mathrm{59}$
$\mathrm{L}$

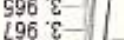

786 '

$016.0-$

Ett t $=$

$0<0$

$\angle t 0^{\circ} 9-$

$010^{\circ} 9$

2809

\begin{tabular}{l}
6679 \\
689 \\
\hline 5
\end{tabular}

128.9

ᄂ28 ' 9

BE8 9

I 98.9

$09 Z 1-$

SG ?

662

018.

tie 5

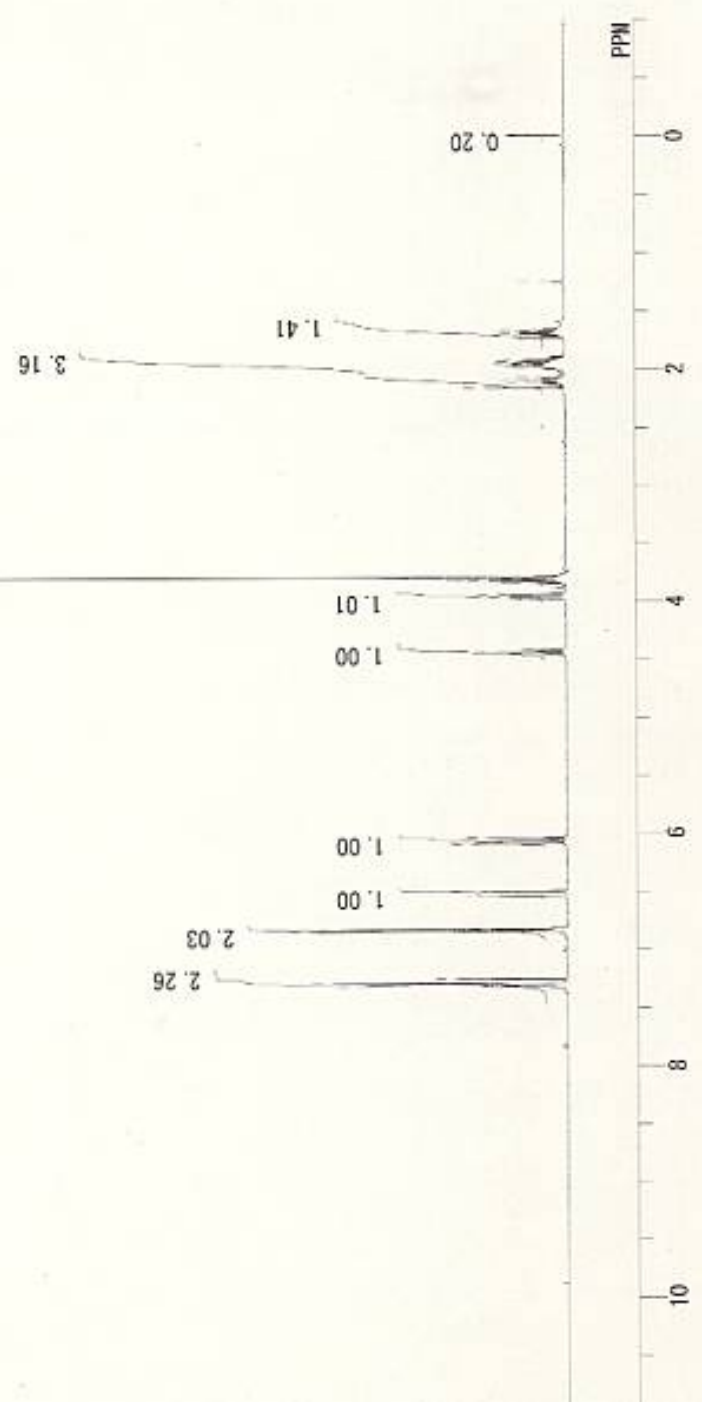




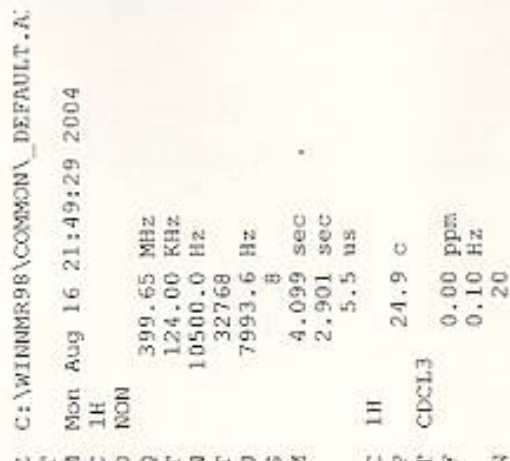

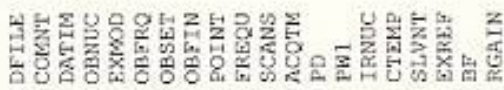

$600.0-$

$000^{\circ} 0$

5द2:

$499^{\circ}=$

$4: L^{\circ} \mathrm{I}$

GदL

cel.

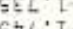

$8 B L \cdot T$

$99 L^{\circ}$

$796.5-1$

$756^{\circ} \mathrm{T}$

$0 \angle 6^{\circ} \cdot \mathrm{T}$

EL6. T-

$686^{\circ} \mathrm{T}-$

I5 $55^{\circ} \mathrm{I}-$

$100^{\circ} \mathrm{z}$

$2 Z 0^{\circ} z$

ยโT:z-

$92 \mathrm{~T}^{\circ} \mathrm{z}-$

OEI'?

हDt.

0 ST $\cdot 2$

LST: 2

E9t- 2

$89: 2$

BLT'Z-

$\angle 20^{\circ} \cdot$

$260^{\circ} \varepsilon$

$4.78^{\circ}$

$998 \cdot \varepsilon-1=$

$288^{\circ} \varepsilon-$

256.

$696^{\circ} \cdot \frac{8}{8}$

$685^{\circ} 8$

$686^{\circ} 8-$

$100^{\circ} \mathrm{b}$
$\angle B 0^{\circ} \mathrm{H}$

Dog' 0

$912 \% 9$

t62.9-

st? $9-$

088.9

$865 \cdot 9$

EEg.9

$\forall \subseteq Z^{\circ} \mathrm{L}-$

$900^{\circ} \mathrm{L}$

$\angle 96^{\circ} \mathrm{L}$

$\angle E S^{\circ} \mathrm{C}$
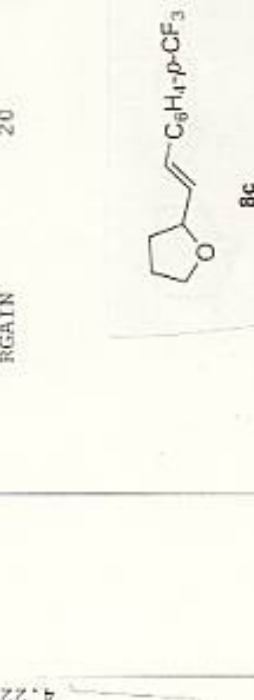

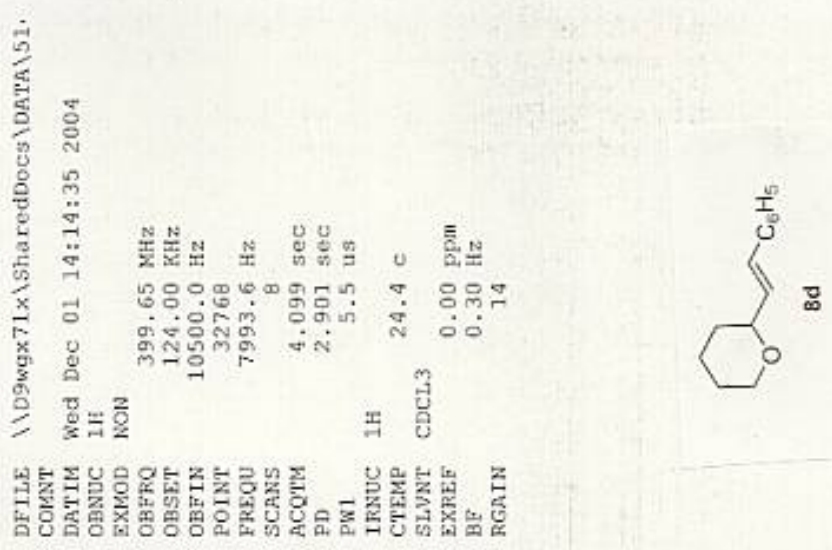

$768^{\circ} \mathrm{I}-$

II ${ }^{\circ}$.

$\angle I b^{\circ}$

bob 1

2S6. T-

$286^{\circ} \mathrm{T}$

167.

हTS: :-

I25.

ess.

Lรद

ह5.

$599^{\circ} \mathrm{T}-$

-

$608^{\circ}$

$828^{\circ} \mathrm{I}$

टहЯ'

$0>8^{\circ} \mathrm{T}-$

$8 \varepsilon \gamma^{\circ} \varepsilon-7=$

$497^{\circ} \mathrm{E}$

$\varepsilon \angle b \cdot \varepsilon-F$

$567 \cdot \varepsilon-1$

$205^{\circ} 8-$

$668^{\circ}$

668.8

$675^{\circ}$

$616 \cdot \varepsilon$

$086^{\circ} \varepsilon-$
$586^{\circ} \varepsilon$

800.7

810.7

$907 \cdot 9$

0zt.9 $=$

$90 \mathrm{~T}$

T97'9

$986 \cdot 9$ F

$425 \cdot 9$

Lat. 2

6ZT:L-

OहT. -

$05 t^{\circ} \mathrm{L}$

est $L$

$955^{\circ} \mathrm{L}$

$6 L T^{\circ} L$

$\angle 6 T^{\circ} \cdot \mathrm{L}$

हT2. $L$

$\angle\left[2^{\circ}\right.$

$0 \times 2^{\circ}$

$5 E 2 \cdot$

$\angle B Z^{\circ} \cdot 4$

SOE $L$

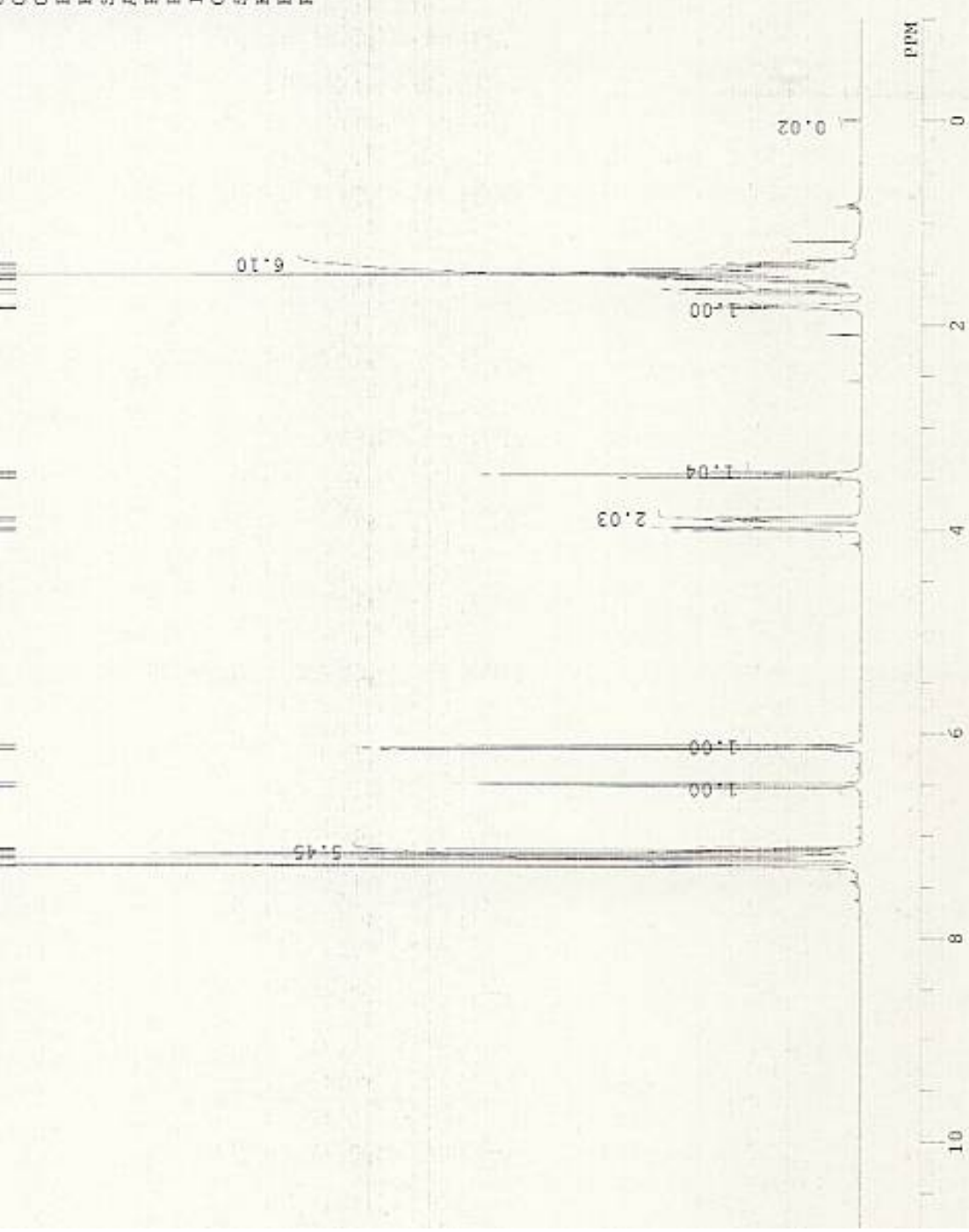




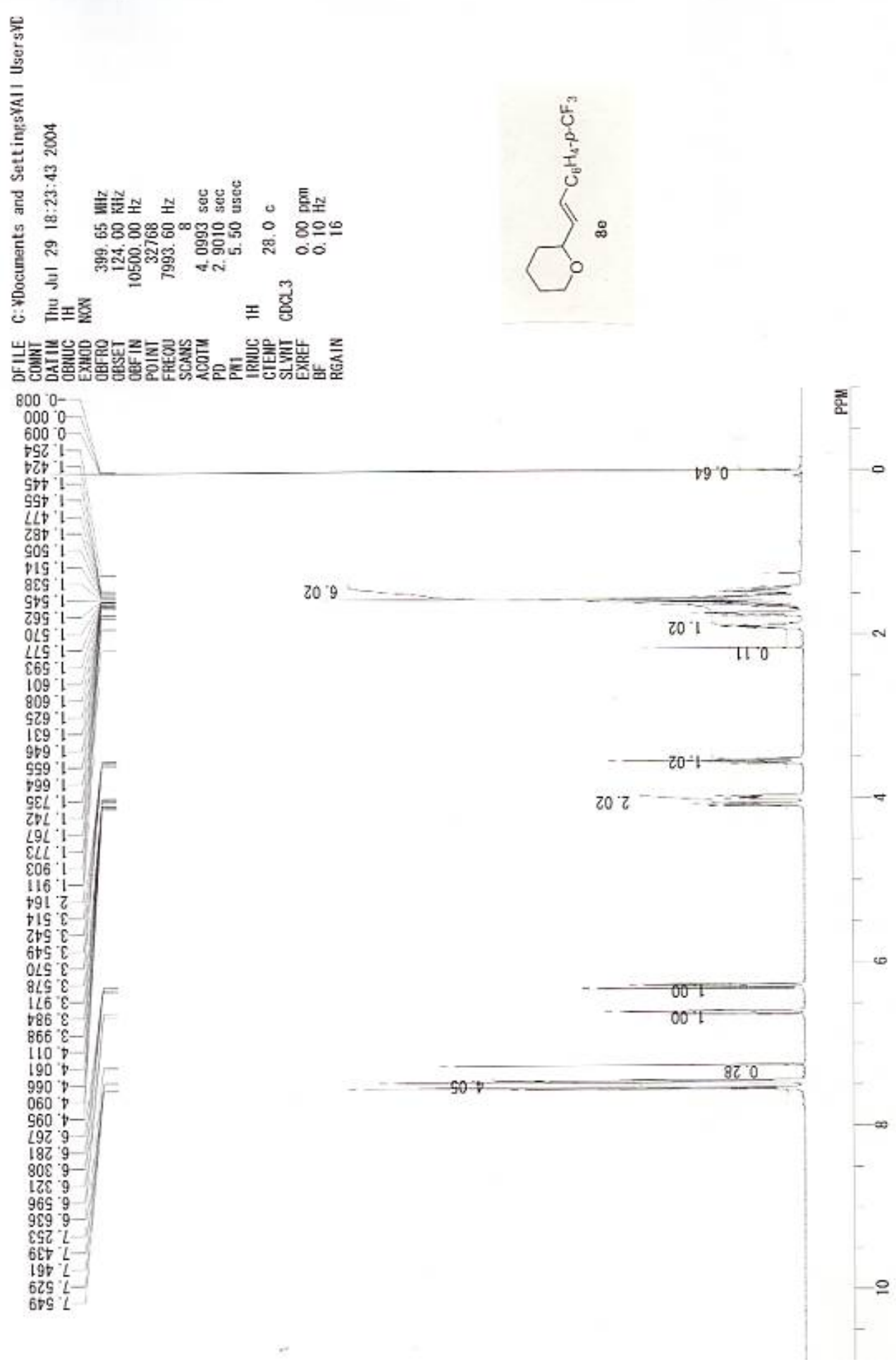



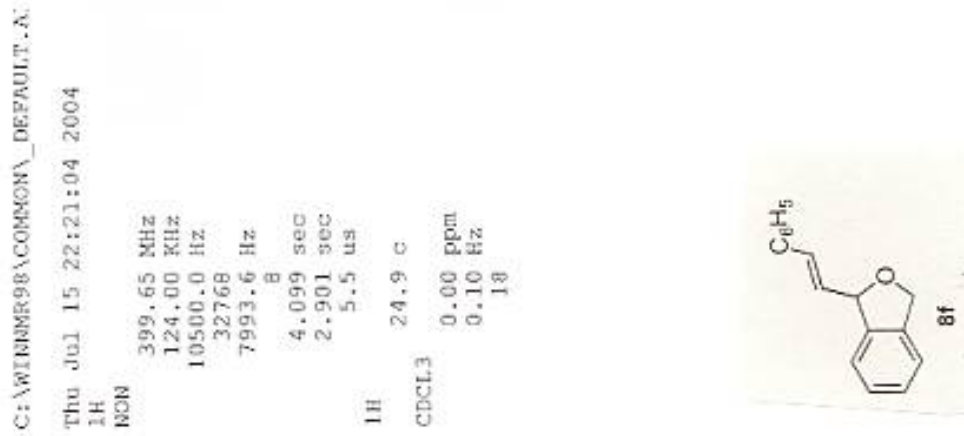

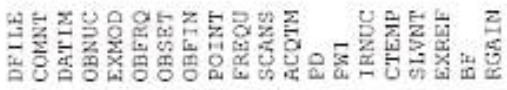

$000^{\circ} 0$

$6>5+$

$997 \cdot 2$

8т7:

8DI:5-

$412+5$

20ट 5

$\angle 62: 5$

$092^{\circ} 9-$

521.

tse.

$242 \cdot 9-$

$262 \cdot 9$

zte.9

$62 L=9$

$69 L^{\circ} 9-$

$66 T^{\circ} \mathrm{L}$

$6 \mathrm{tz}-2$

LEZ $L$

$672 \div 4-$

$\operatorname{agc} \cdot 2$

$0422^{*} 2$

$282^{\circ} \mathrm{L}-$

$\angle 82^{\circ} \mathrm{L}$

$908 \cdot 2$

6 te 2

उटह $L$

96ह :

$916^{\circ} 2$

ozti 

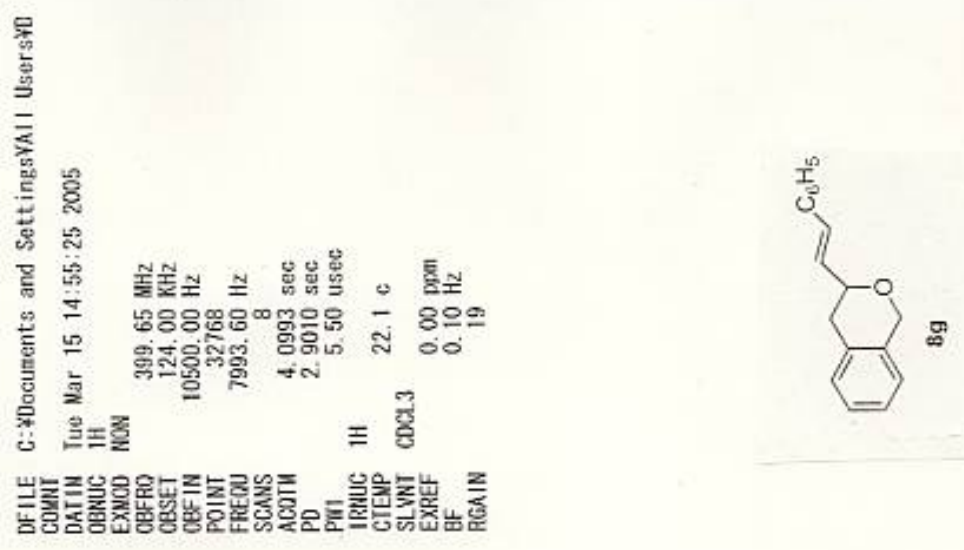

$000 \%$
$020^{\circ}$

Es? I

$\cos 1$

$0<12$
$578 ?$

$798 \%$

988 '

968 '

826 ' 2

996 '

क9:

จLE

L $2 E, b$

$000 \mathrm{D}-\mathrm{C}=$

olt t

$\angle 860^{\circ}$

GL6:

Lto -9

$19 \varepsilon^{-9}$

$286-9$

$\varepsilon 69^{\circ} 9$

ZEL" 9

G10 2

$\angle 2 O L$

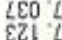

$981-2$

901 F

$8911-$

EL.

181.

$561 \div$

$928: 2$

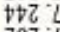

292 2

COE 2

$2 Z E^{\prime} L$

$O \forall E 2$

$\mathrm{COS}^{2} \mathrm{C}$

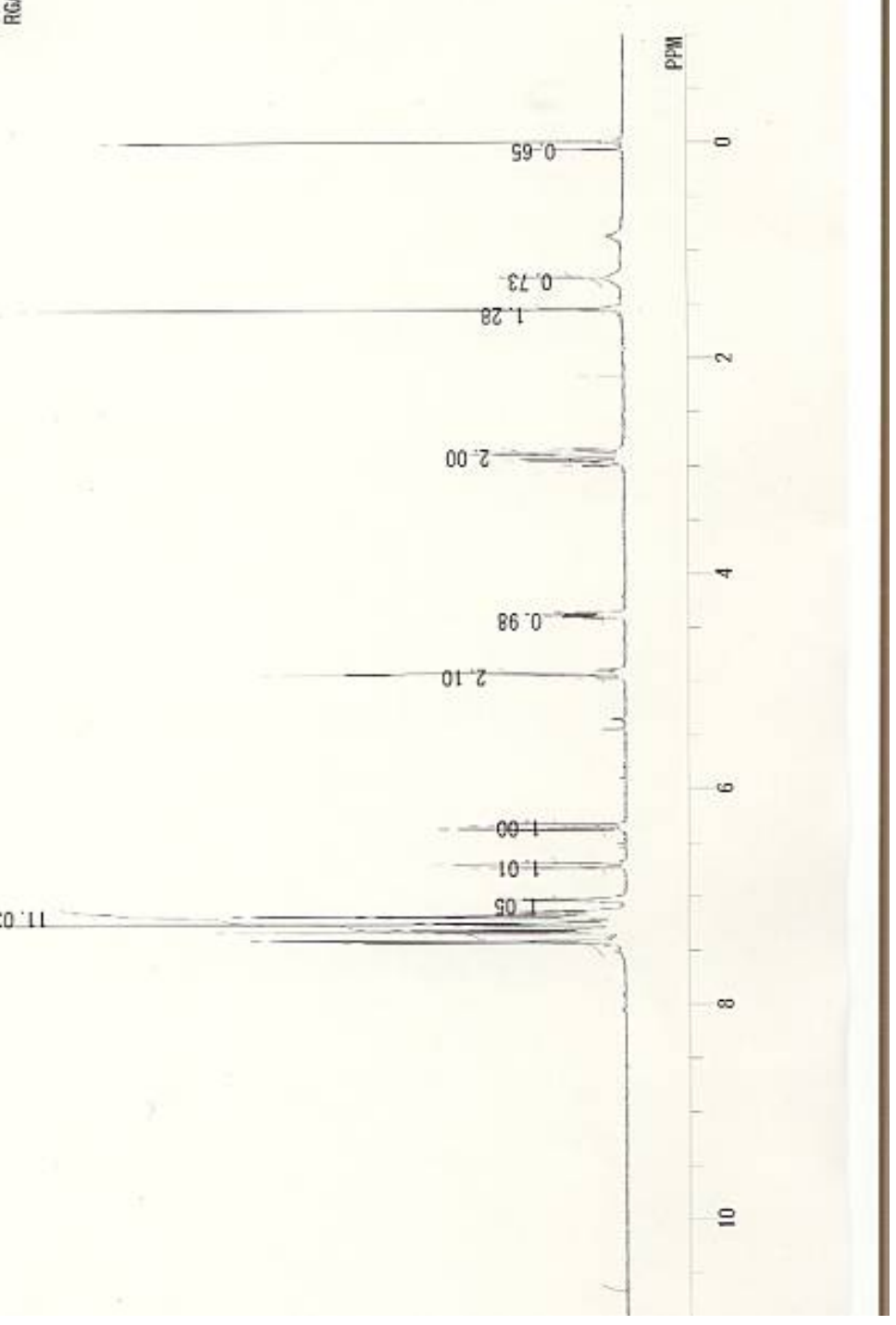



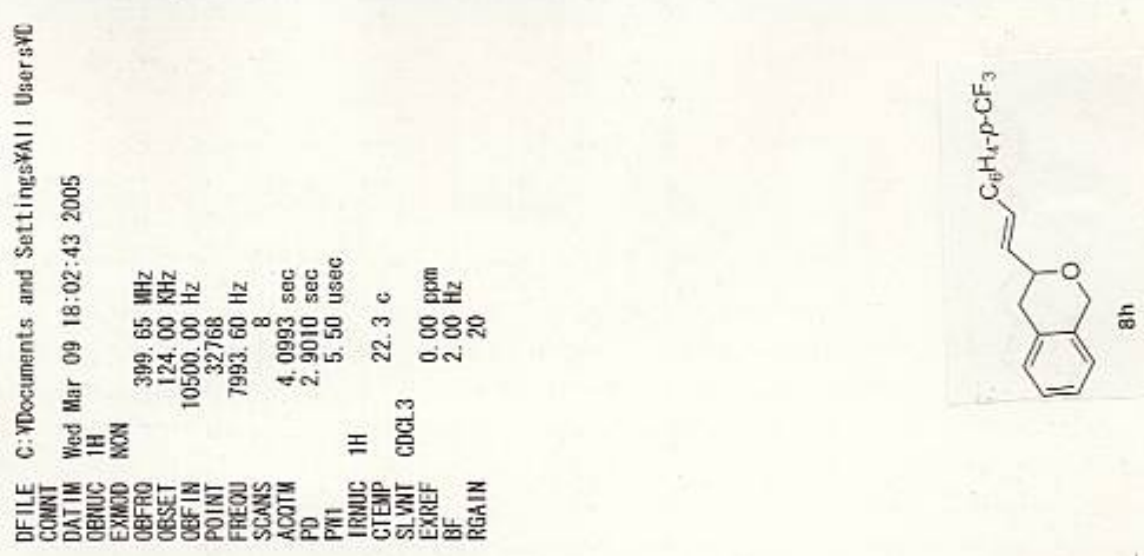

$000 \%$

8891

$04: 2$

9982

$468^{2} Z$

$9062=$

$826 ?$

$6066^{\circ} 2$
$686^{2} 2-$

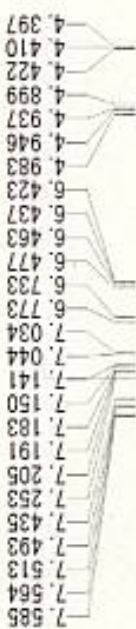

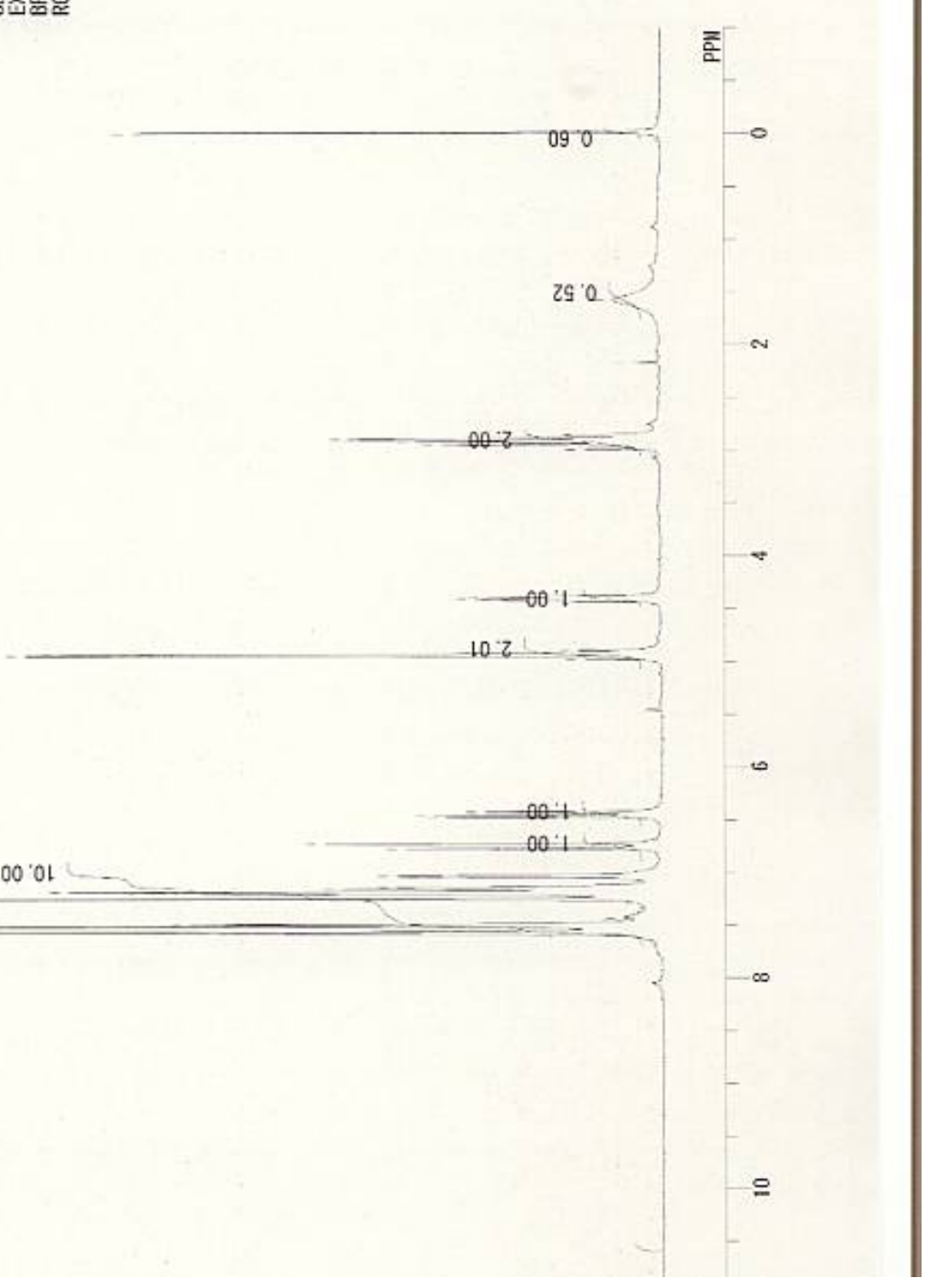



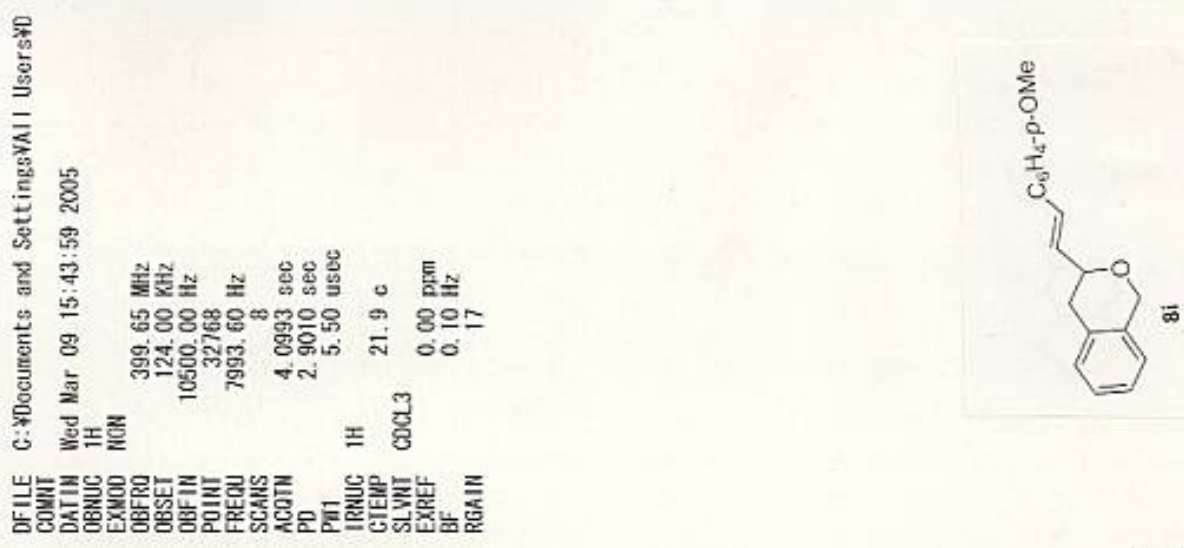

$000^{\circ} 0$

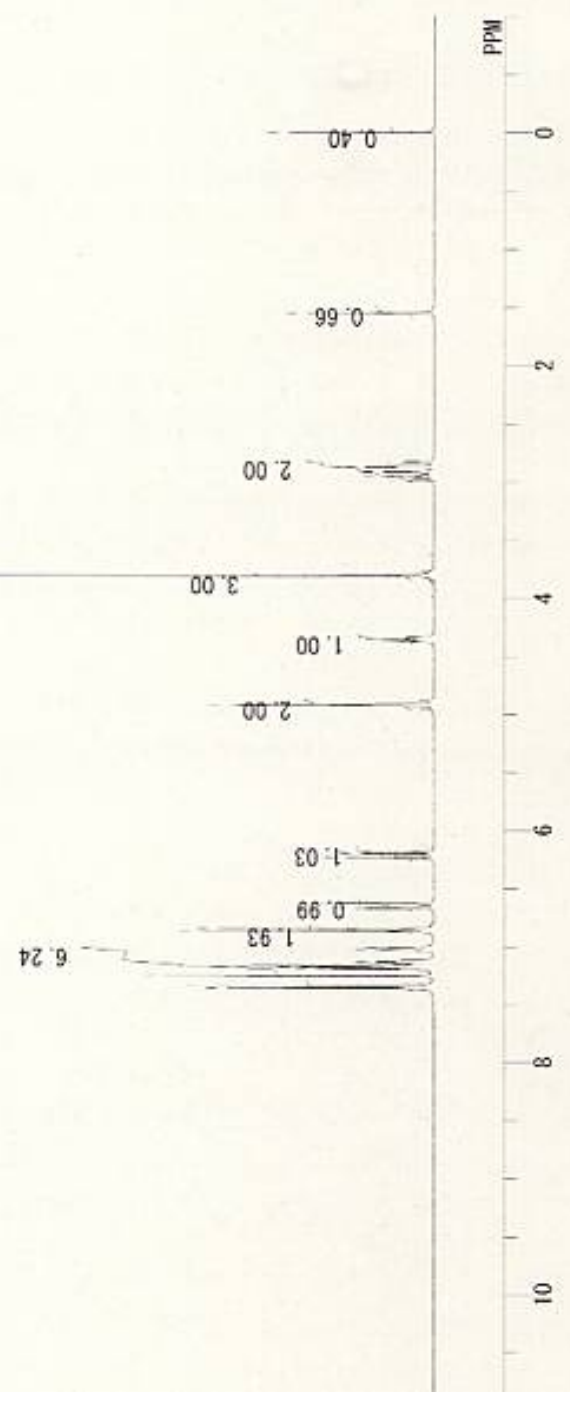




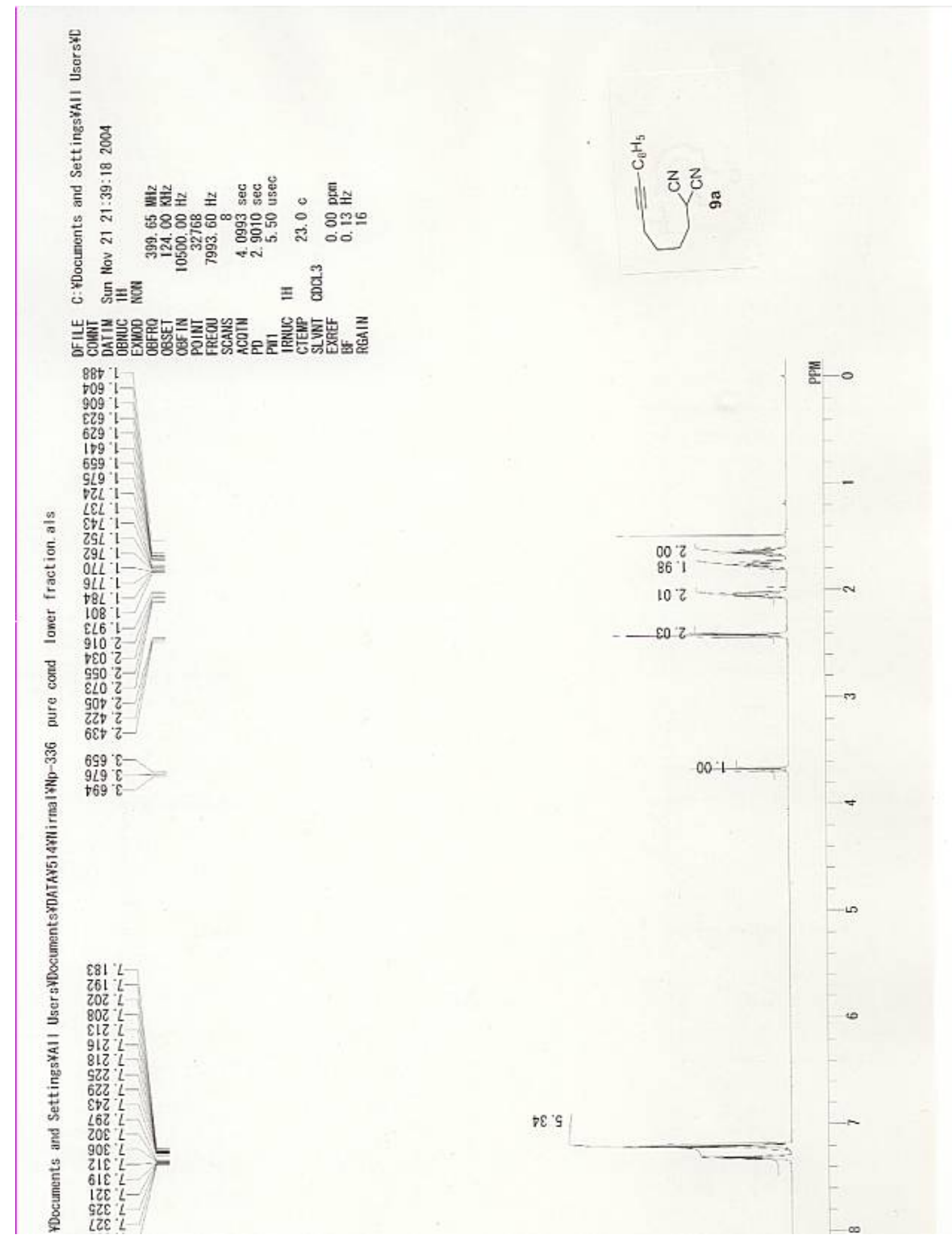



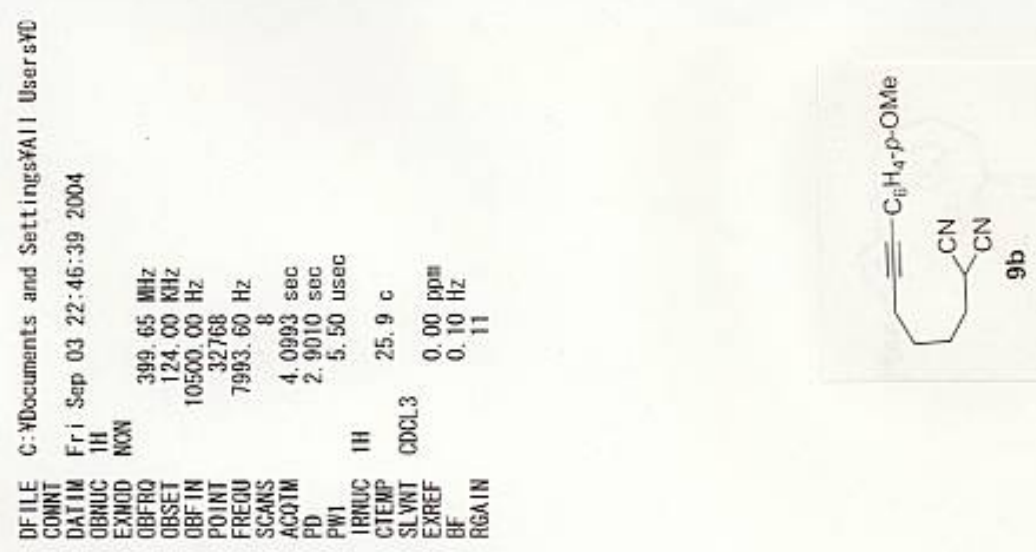

$000^{\circ} 0$
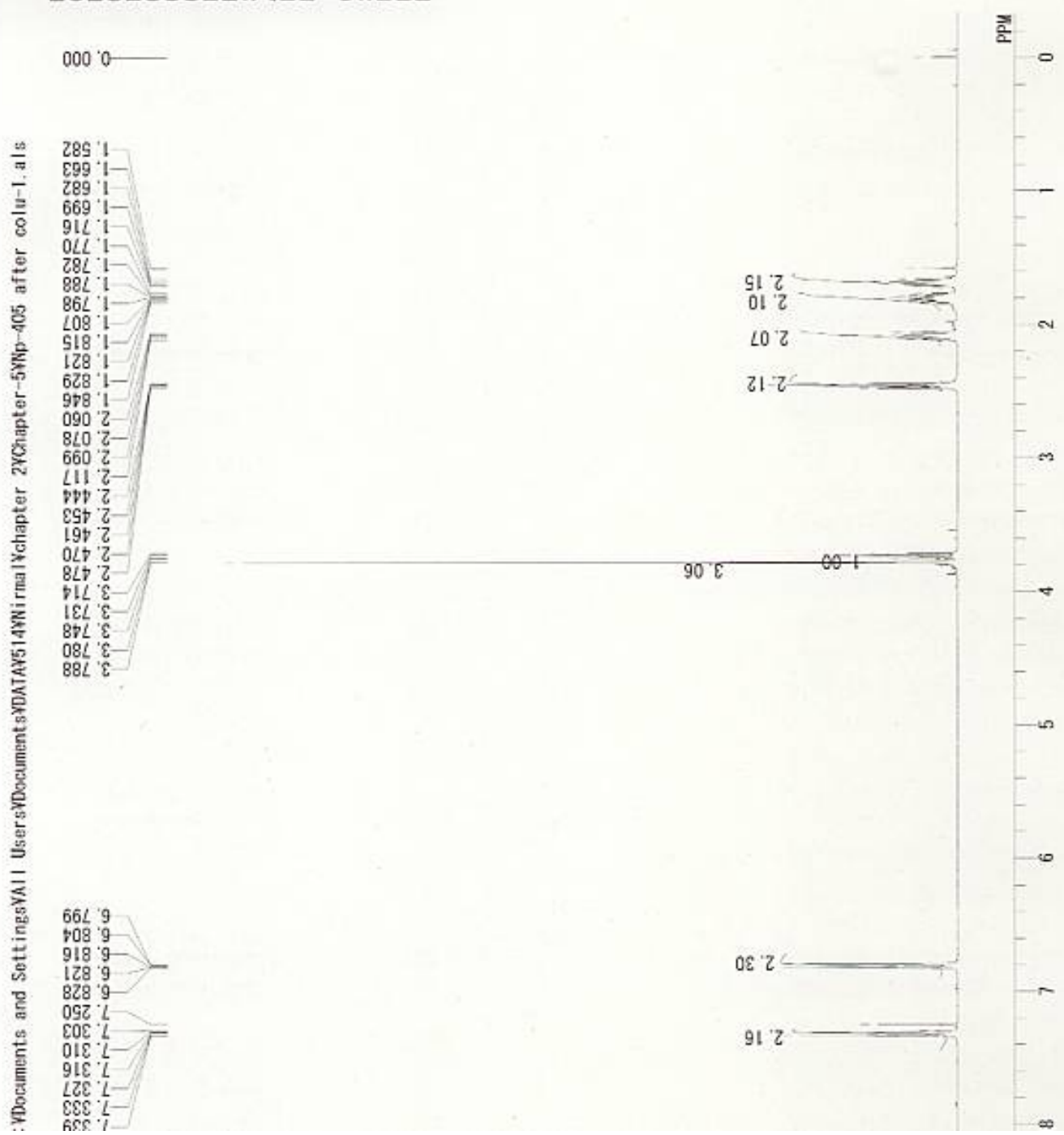

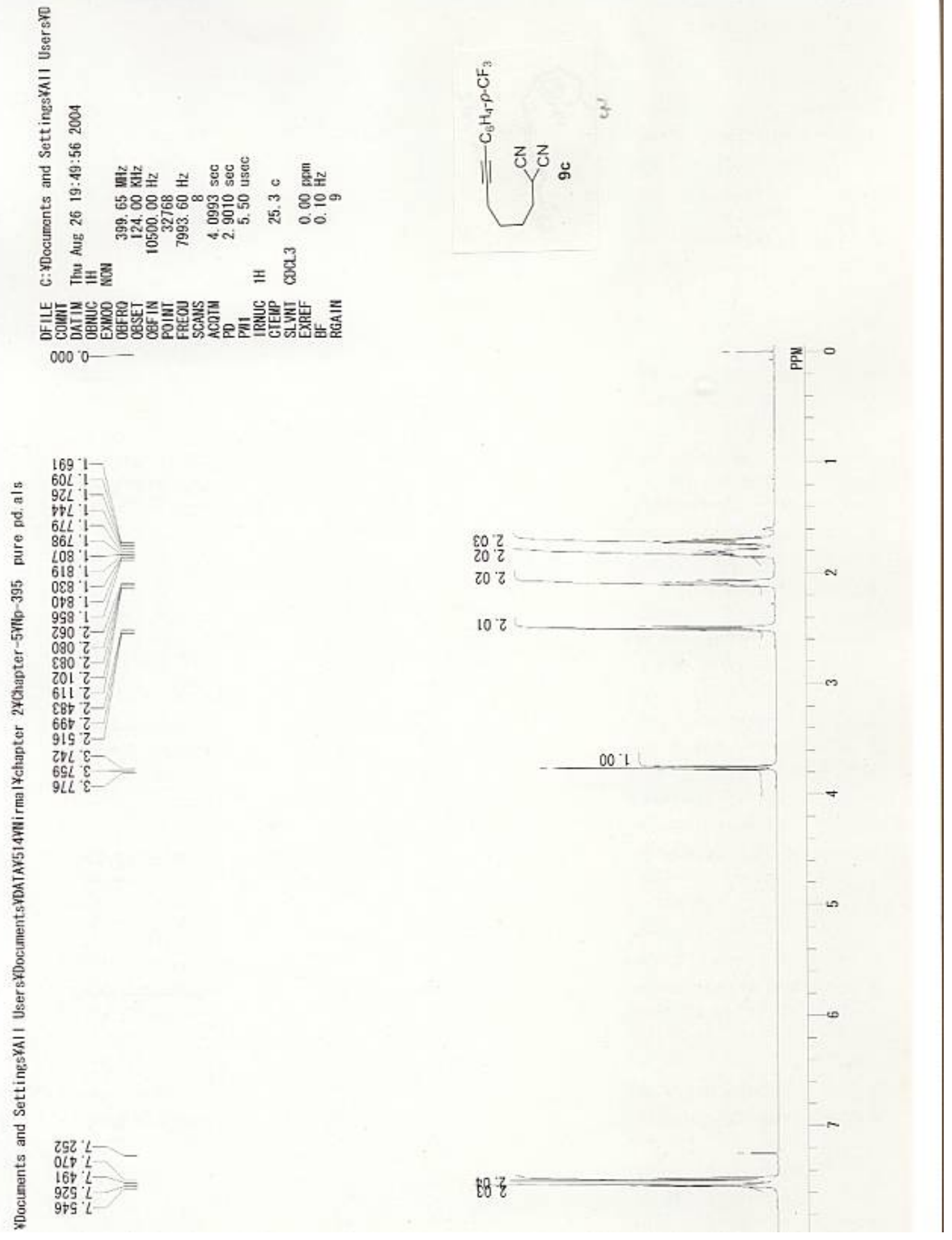

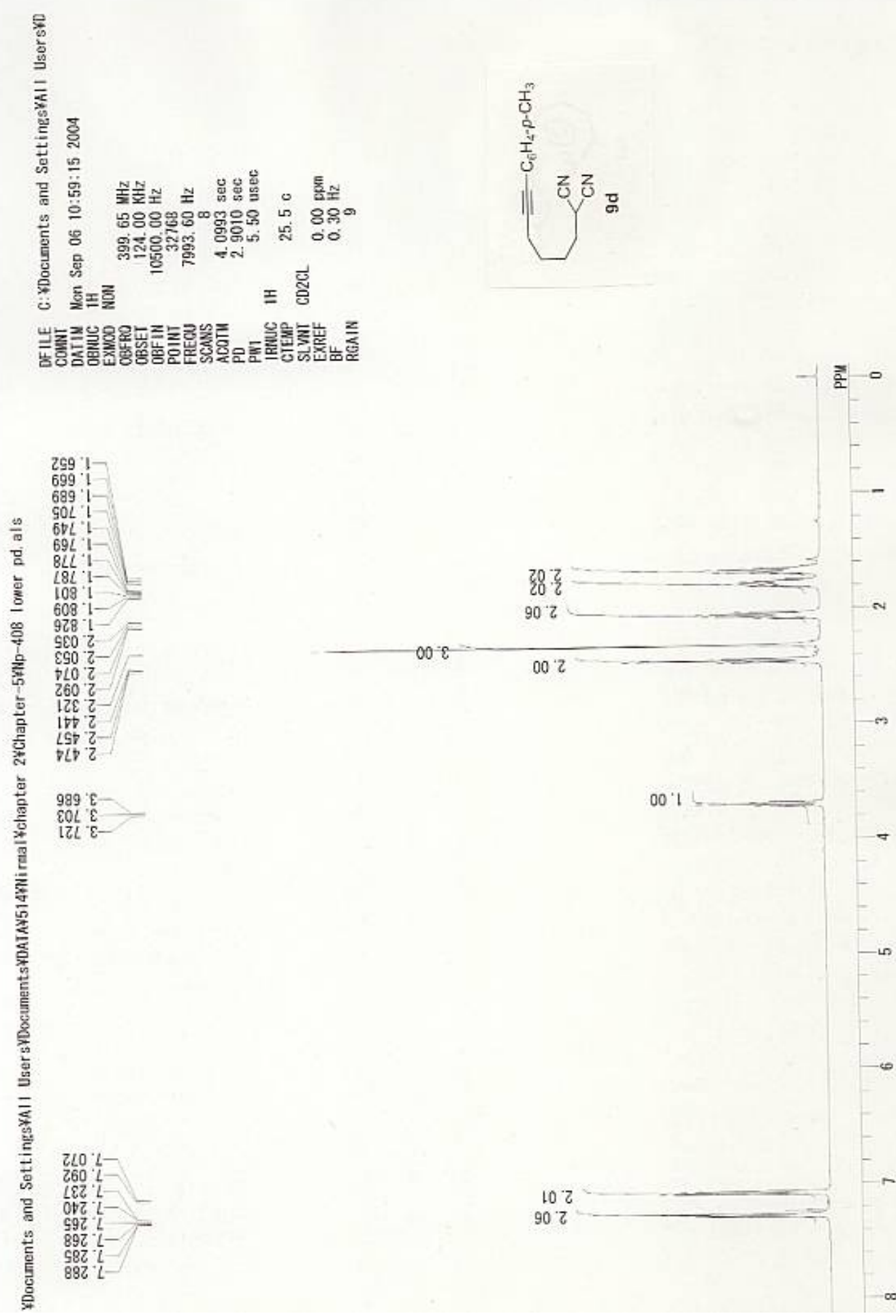

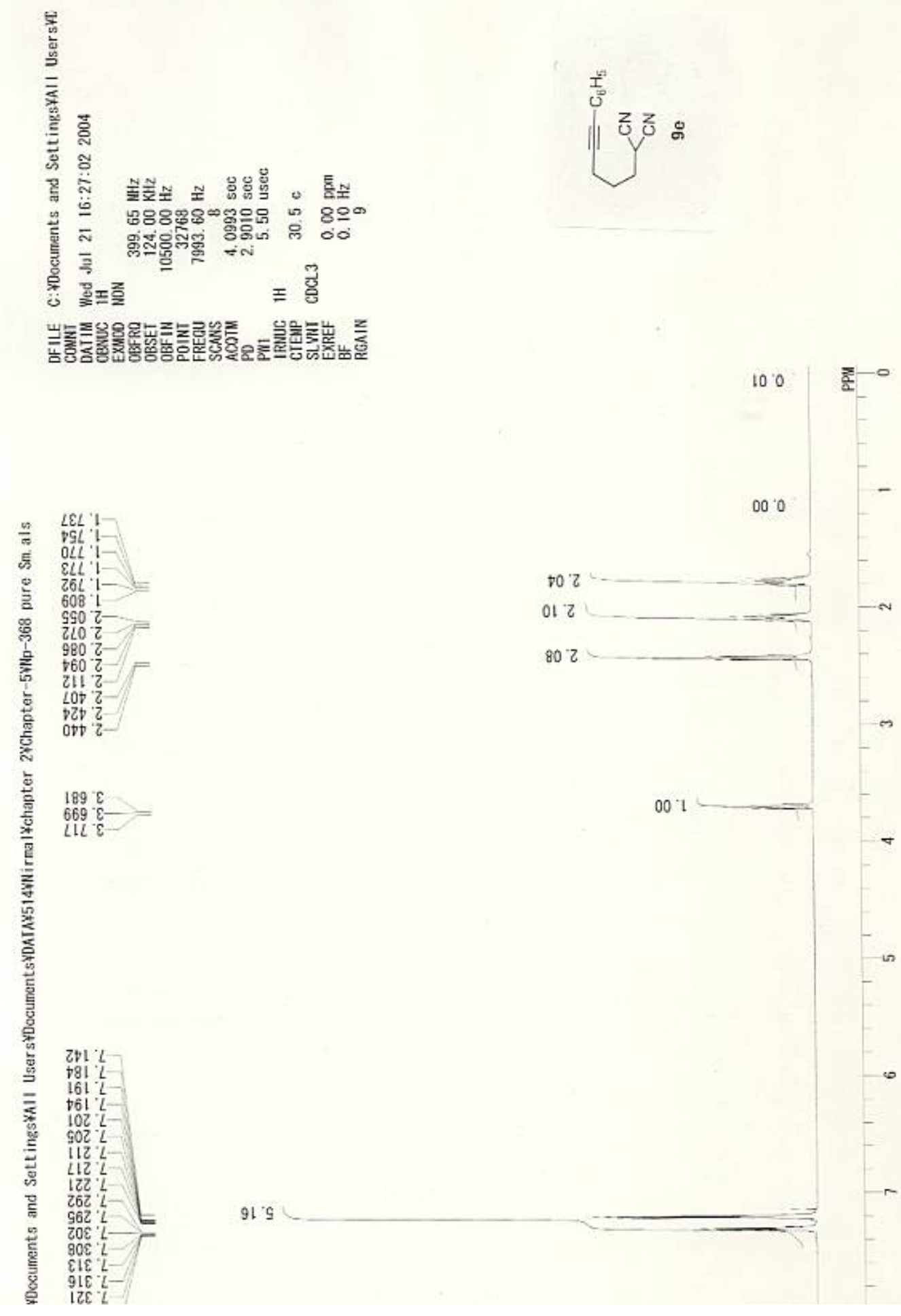


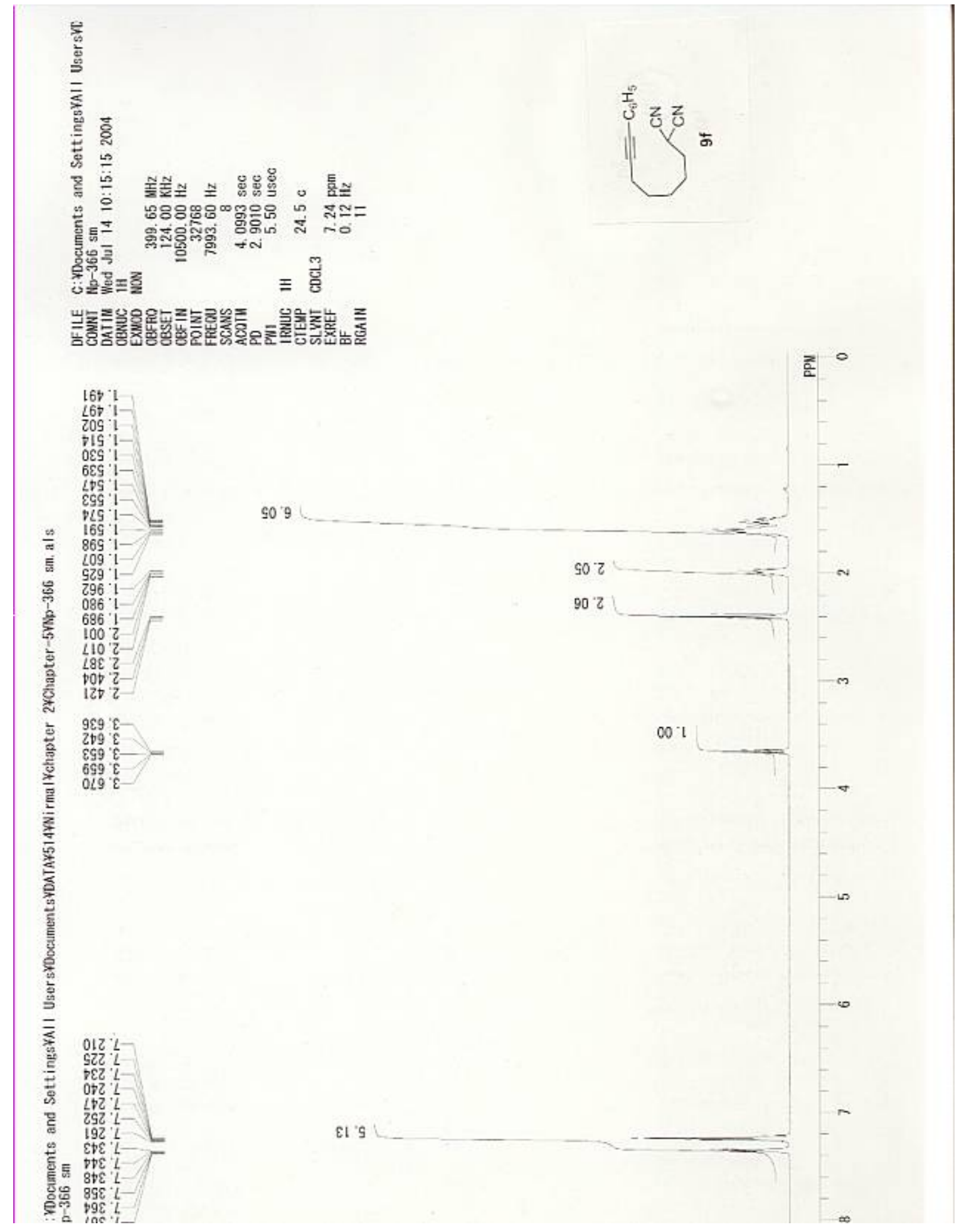




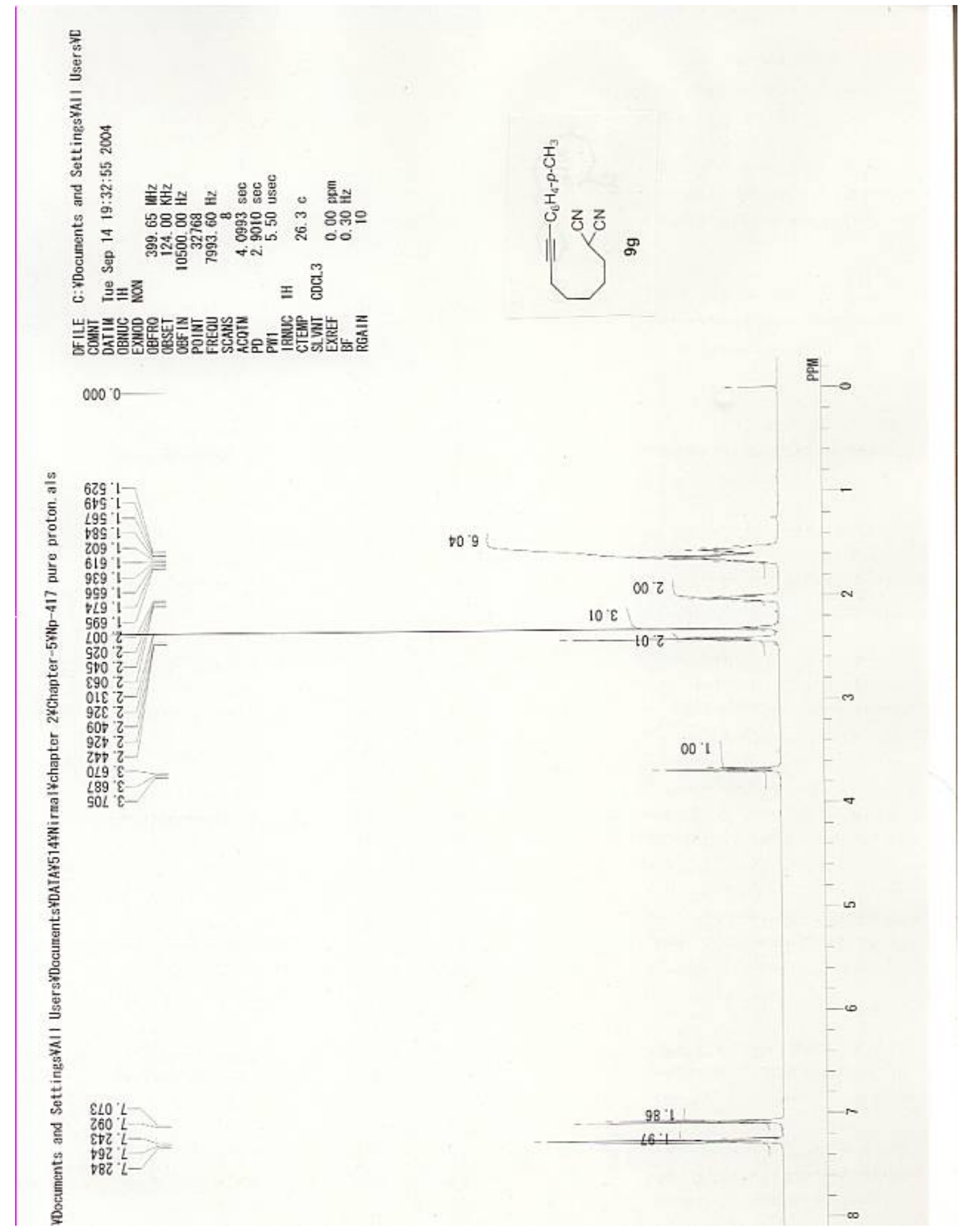




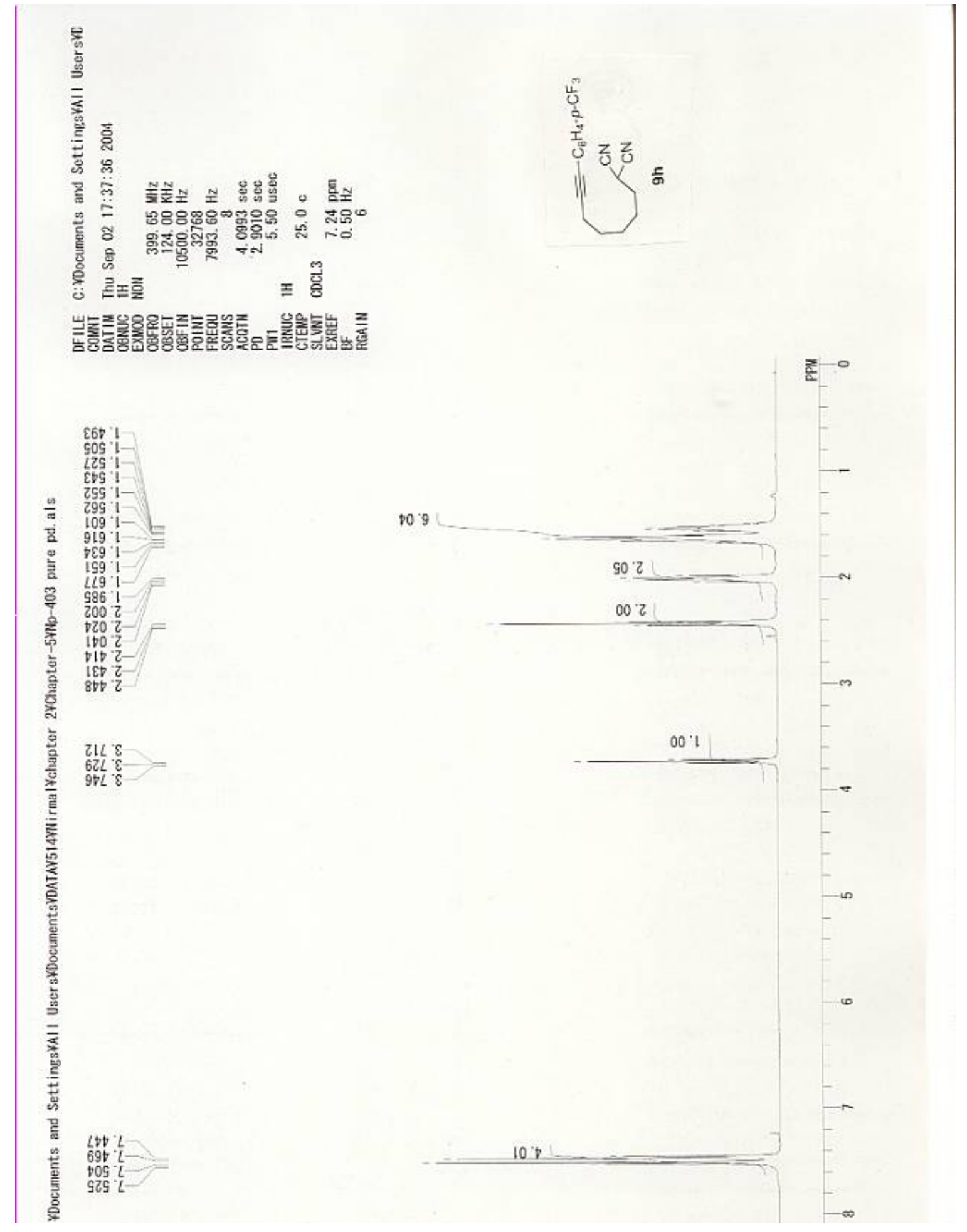



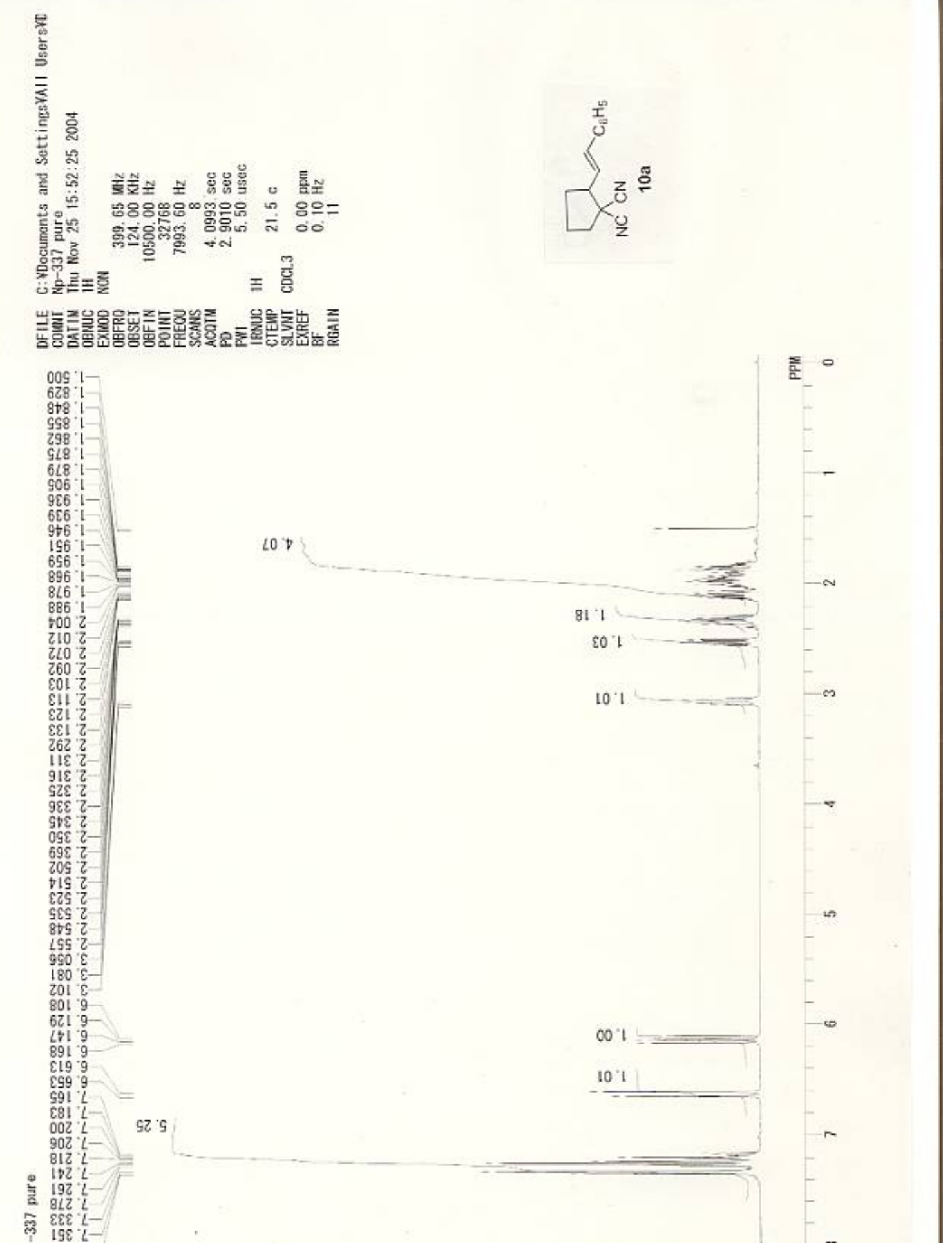


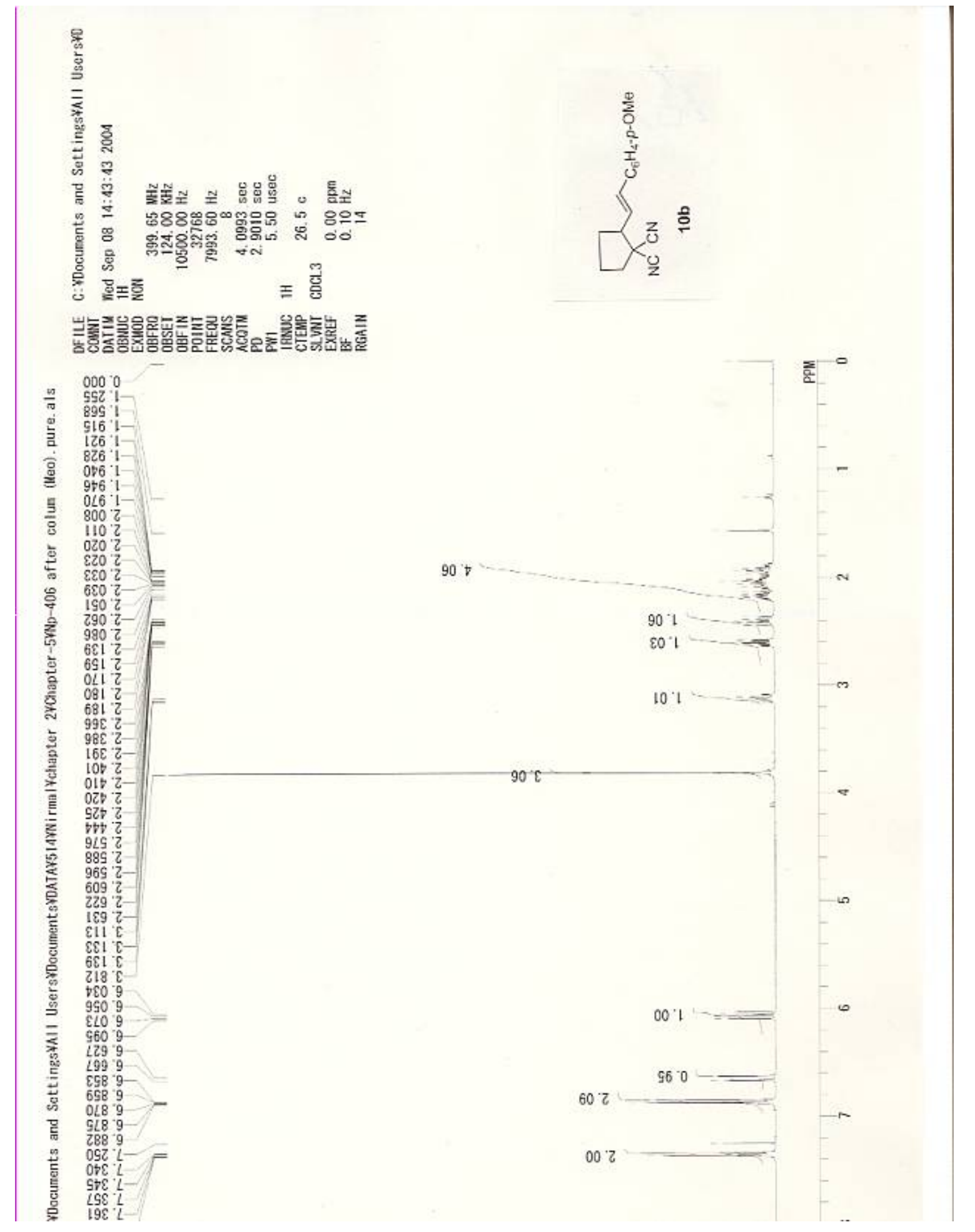




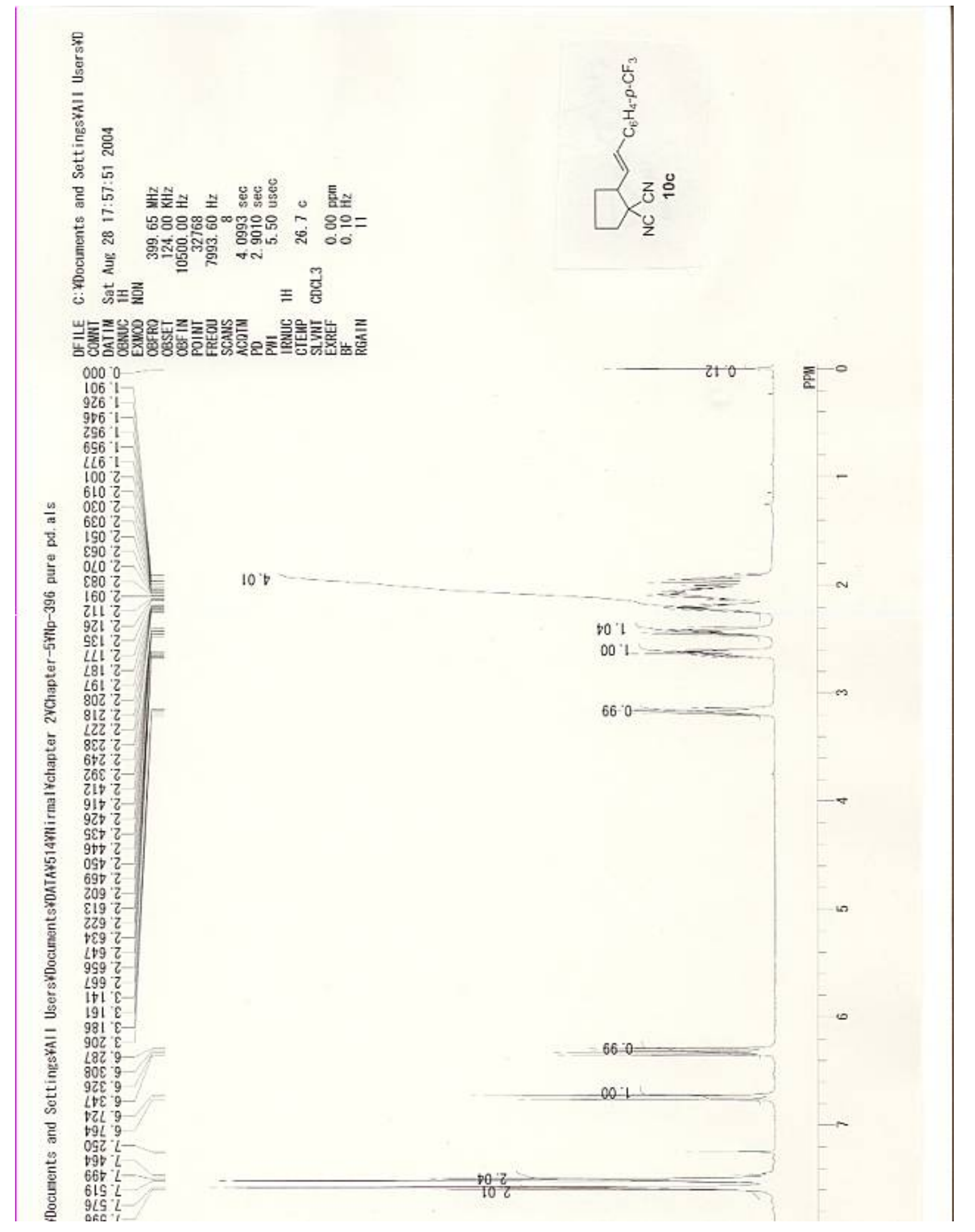




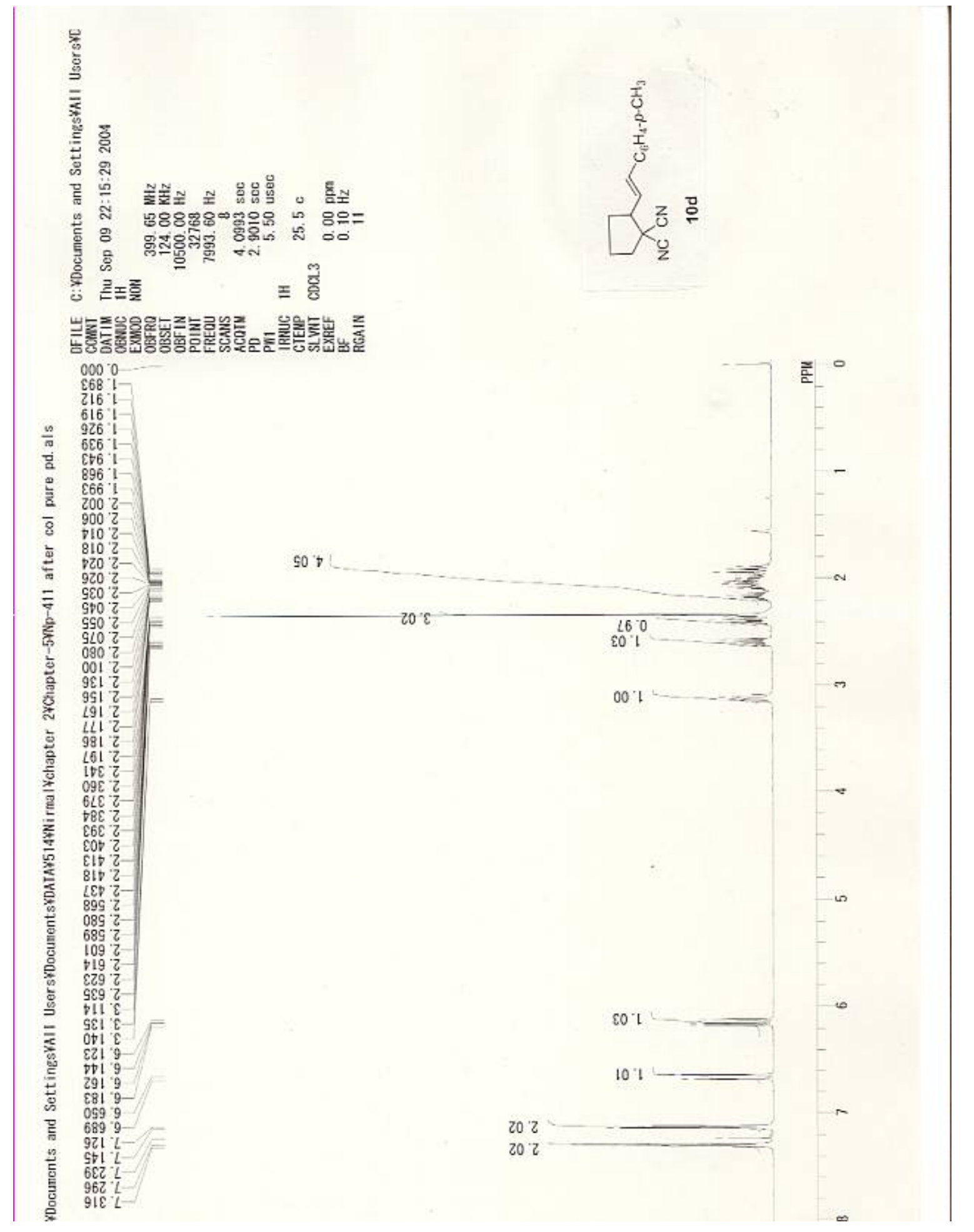




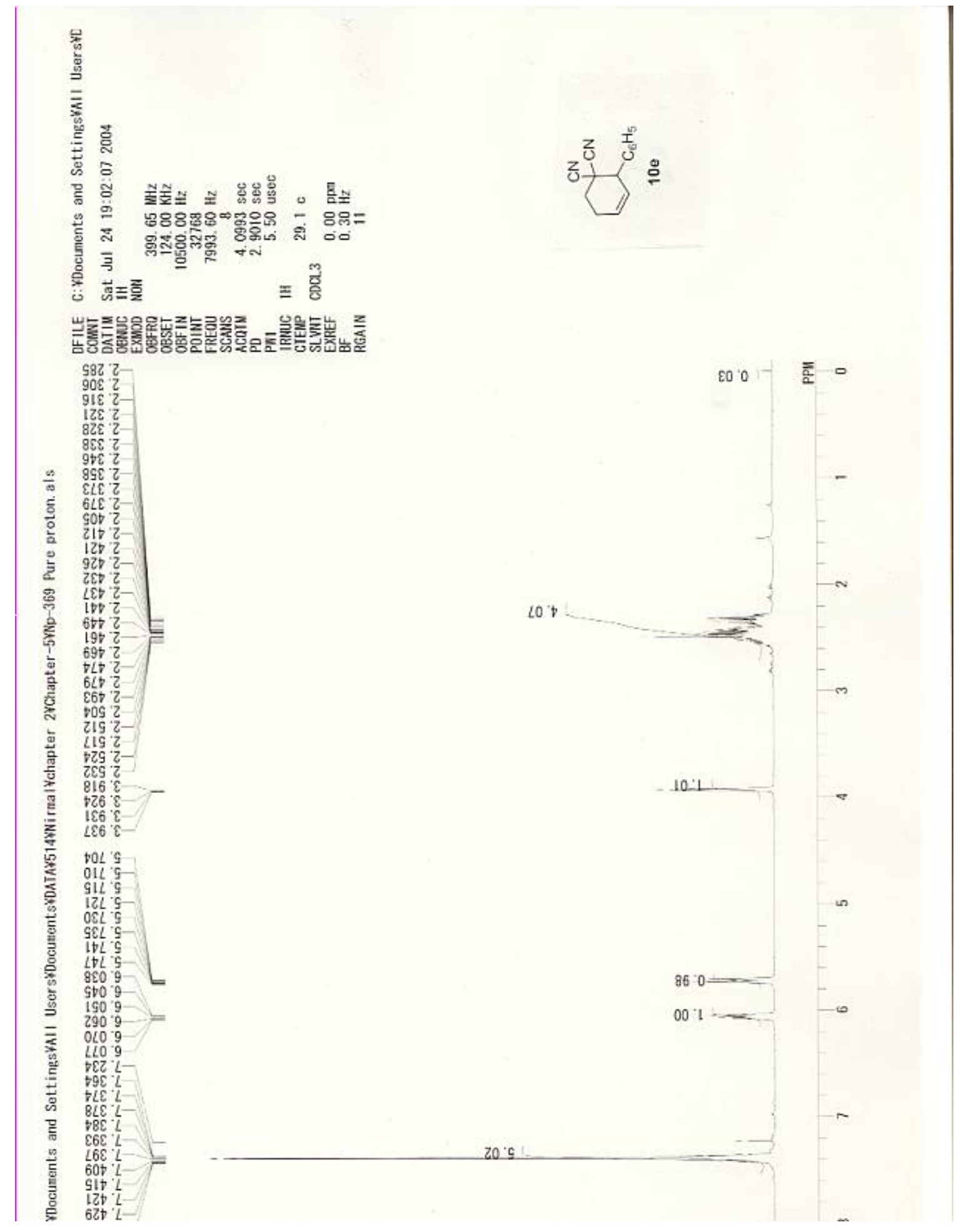




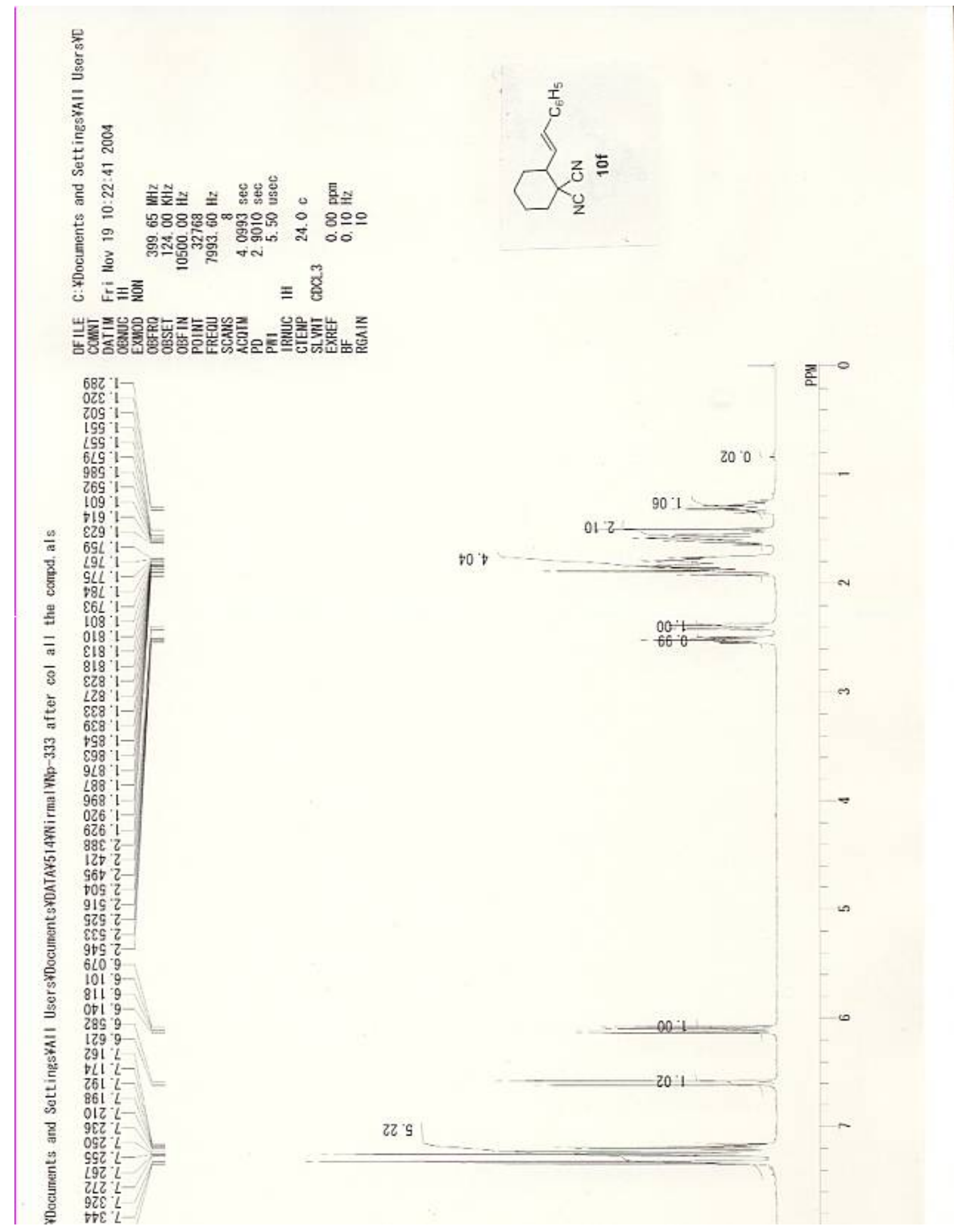




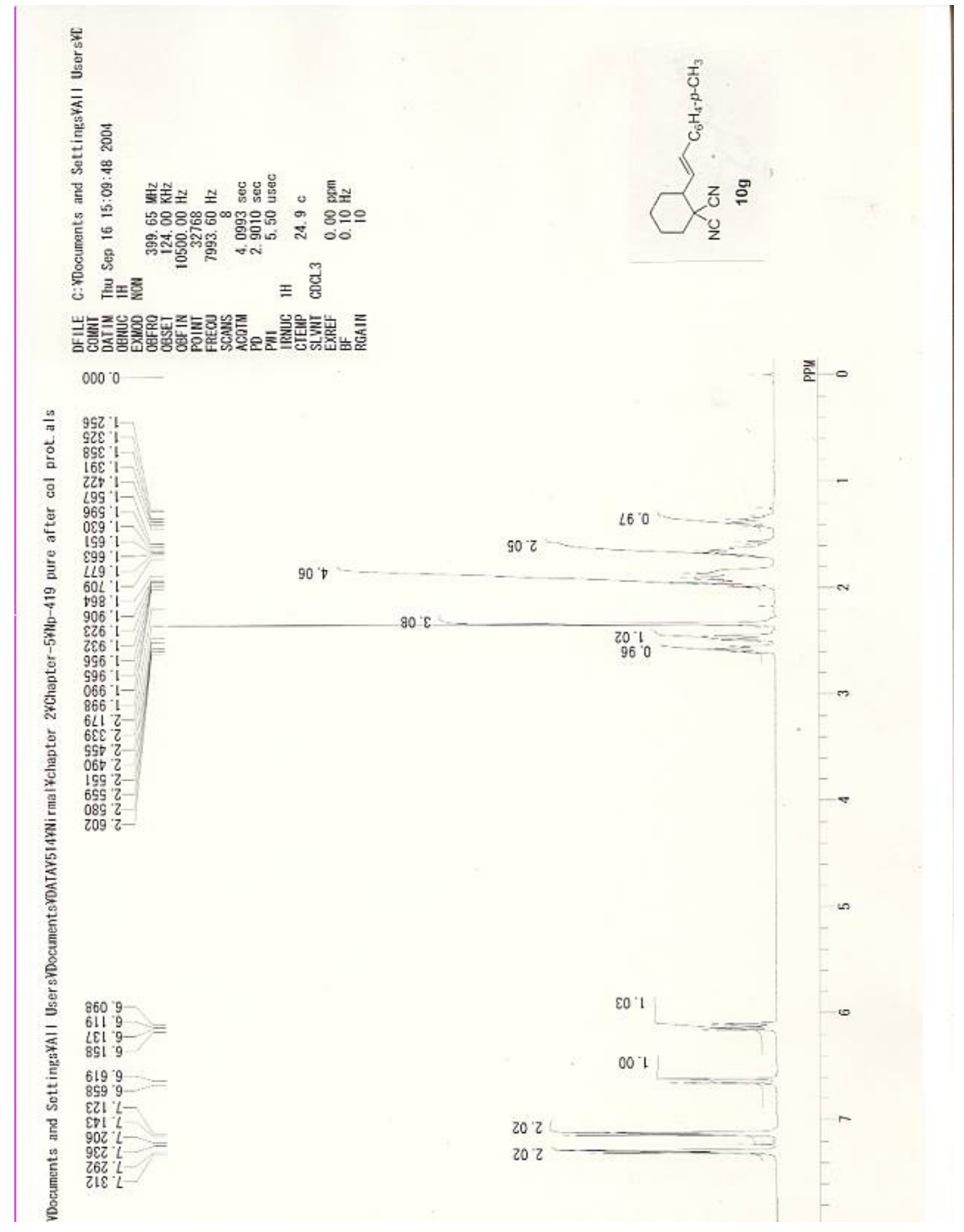




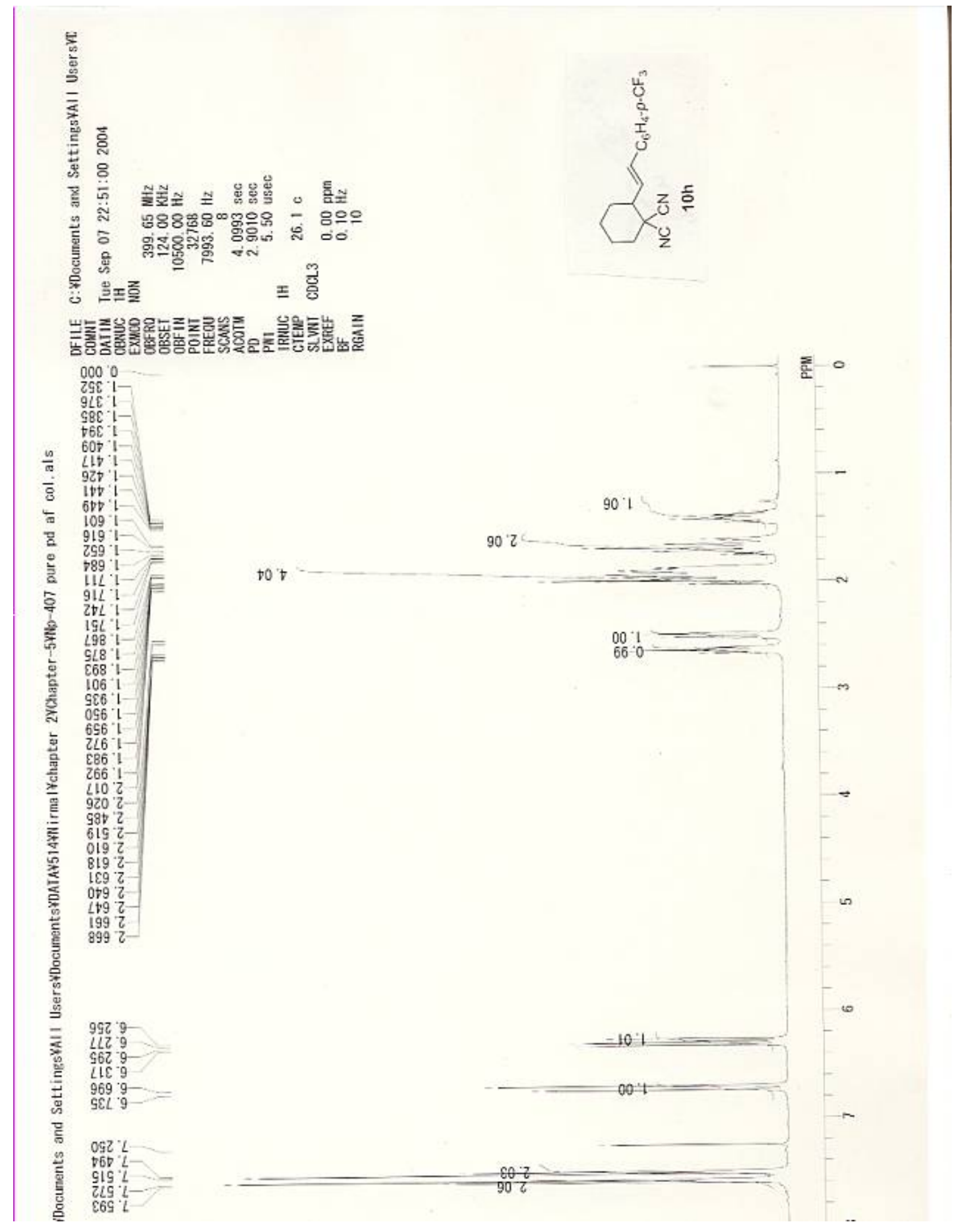




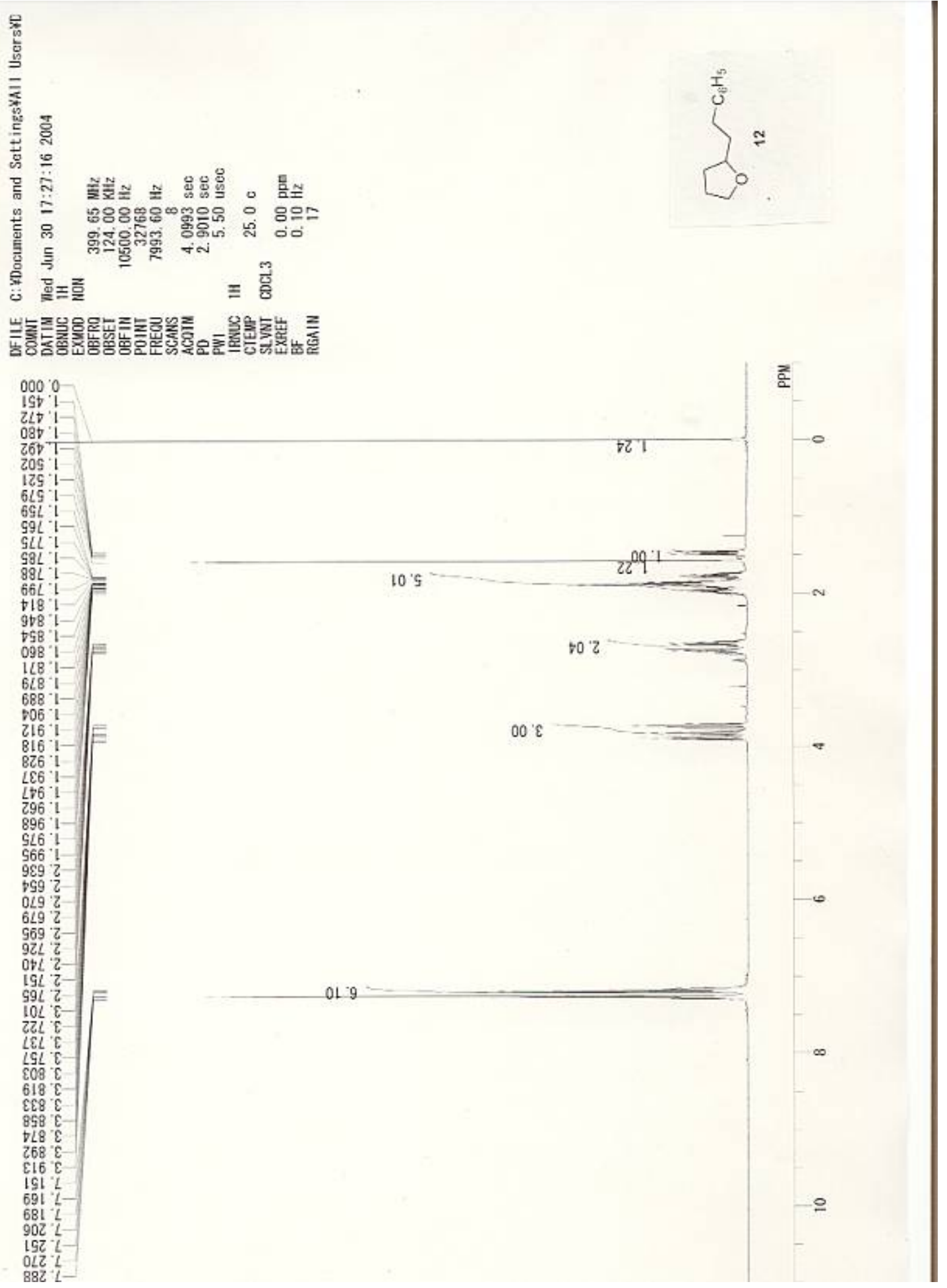




\section{Cartesian Coordinates}

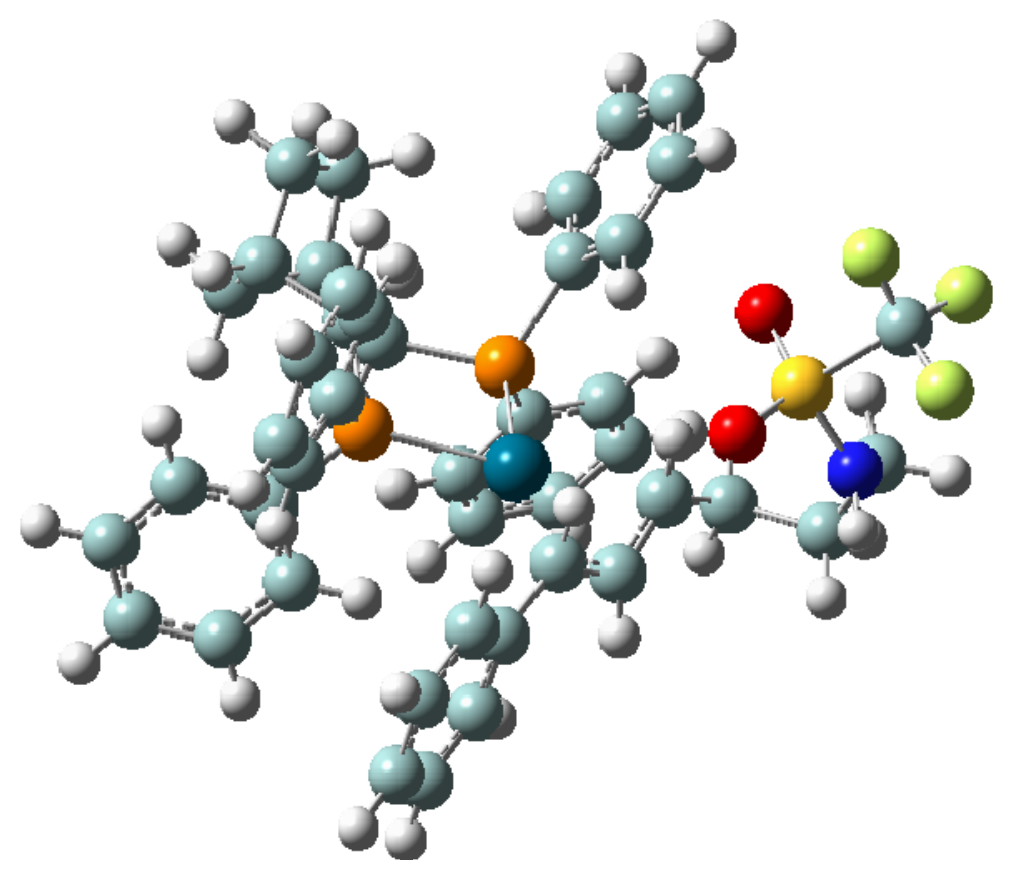

$\pi$-Allyl intermediate in hydroamination, isomer I

$\mathrm{E}(\mathrm{RB}+\mathrm{HF}-\mathrm{LYP})=-3416.61274125 \quad$ A. U.

Standard orientation:

\begin{tabular}{rccccc} 
Center & Atomic & Atomic & \multicolumn{3}{c}{ Coordinates (Angstroms) } \\
Number & Number & Type & X & Y & $Z$ \\
------1 & & & \\
1 & 6 & 0 & -2.540749 & 3.444527 & 2.912290 \\
2 & 6 & 0 & -3.172421 & 3.101594 & 1.525764 \\
3 & 6 & 0 & -3.450956 & 1.181603 & 2.750033 \\
4 & 6 & 0 & -2.829236 & 2.165783 & 3.785165 \\
5 & 1 & 0 & -1.473358 & 3.666459 & 2.847247 \\
6 & 1 & 0 & -3.031815 & 4.328824 & 3.334092 \\
7 & 1 & 0 & -1.924527 & 1.765854 & 4.258194 \\
8 & 1 & 0 & -3.549224 & 2.383529 & 4.582663 \\
9 & 6 & 0 & -4.354886 & 2.167552 & 1.950388 \\
10 & 1 & 0 & -4.884181 & 1.716390 & 1.104534
\end{tabular}




\begin{tabular}{|c|c|c|c|c|c|}
\hline 11 & 1 & 0 & -5.085261 & 2. 670034 & 2. 593921 \\
\hline 12 & 6 & 0 & -2.352576 & 2. 037105 & 0.738303 \\
\hline 13 & 1 & 0 & -2.997900 & 1. 737538 & -0.100470 \\
\hline 14 & 6 & 0 & -2.290228 & 0.847537 & 1. 750623 \\
\hline 15 & 1 & 0 & -1.339386 & 0.879337 & 2. 298839 \\
\hline 16 & 1 & 0 & -3.444225 & 3. 974564 & 0.926022 \\
\hline 17 & 1 & 0 & -3.941866 & 0.314513 & 3. 197505 \\
\hline 18 & 15 & 0 & -2.135739 & -0.764043 & 0.752530 \\
\hline 19 & 15 & 0 & -0.679898 & 2. 207176 & -0.148245 \\
\hline 20 & 6 & 0 & -0.989198 & 3. 340320 & -1.606553 \\
\hline 21 & 6 & 0 & 0.016787 & 4. 226250 & -2.052327 \\
\hline 22 & 6 & 0 & -2.201561 & 3. 259624 & -2.328752 \\
\hline 23 & 6 & 0 & -0.193774 & 5. 029372 & -3.188460 \\
\hline 24 & 1 & 0 & 0.956758 & 4. 306417 & -1.513489 \\
\hline 25 & 6 & 0 & -2.410631 & 4. 065878 & -3.462025 \\
\hline 26 & 1 & 0 & -2.991539 & 2. 578026 & -2.024126 \\
\hline 27 & 6 & 0 & -1.408299 & 4. 954791 & -3.896136 \\
\hline 28 & 1 & 0 & 0.586055 & 5. 712751 & -3.514569 \\
\hline 29 & 1 & 0 & -3.351939 & 3. 999918 & -4.001441 \\
\hline 30 & 1 & 0 & -1.572011 & 5. 579129 & -4.770361 \\
\hline 31 & 6 & 0 & 0.555803 & 3. 090948 & 0.935117 \\
\hline 32 & 6 & 0 & 1. 644248 & 2. 361569 & 1. 456670 \\
\hline 33 & 6 & 0 & 0.427344 & 4. 466180 & 1. 238753 \\
\hline 34 & 6 & 0 & 2. 595743 & 2. 995414 & 2. 278959 \\
\hline 35 & 1 & 0 & 1. 774570 & 1. 310309 & 1. 219469 \\
\hline 36 & 6 & 0 & 1. 376796 & 5. 096619 & 2. 061104 \\
\hline 37 & 1 & 0 & -0.399844 & 5. 044948 & 0.835604 \\
\hline 38 & 6 & 0 & 2. 461846 & 4. 361992 & 2.584862 \\
\hline 39 & 1 & 0 & 3. 433662 & 2. 419417 & 2. 660416 \\
\hline 40 & 1 & 0 & 1. 271791 & 6. 153373 & 2. 292345 \\
\hline 41 & 1 & 0 & 3. 195356 & 4. 854342 & 3. 218118 \\
\hline 42 & 6 & 0 & -3.786015 & -1.056774 & -0.067738 \\
\hline 43 & 6 & 0 & -3.884910 & -0.919663 & -1.468271 \\
\hline 44 & 6 & 0 & -4.938715 & -1.376593 & 0.684454 \\
\hline 45 & 6 & 0 & -5.124844 & -1.089709 & -2.112321 \\
\hline 46 & 1 & 0 & -2.995490 & -0.698381 & -2.053645 \\
\hline 47 & 6 & 0 & -6.175953 & -1.548293 & 0.039278 \\
\hline 48 & 1 & 0 & -4.875645 & -1.504129 & 1. 761821 \\
\hline 49 & 6 & 0 & -6.272539 & -1.401787 & -1.359789 \\
\hline 50 & 1 & 0 & -5.191653 & -0.989004 & -3.192502 \\
\hline 51 & 1 & 0 & -7.058406 & -1.796275 & 0.623074 \\
\hline 52 & 1 & 0 & -7.230078 & -1.537329 & -1.855792 \\
\hline 53 & 6 & 0 & -1.923148 & -2.137367 & 1. 997652 \\
\hline 54 & 6 & 0 & -1.189660 & -1.916824 & 3. 184969 \\
\hline 55 & 6 & 0 & -2.426744 & -3.427981 & 1. 724475 \\
\hline 56 & 6 & 0 & -0.979868 & -2.968414 & 4. 094839 \\
\hline 57 & 1 & 0 & -0.778504 & -0.937250 & 3. 415067 \\
\hline 58 & 6 & 0 & -2.217124 & -4.476925 & 2. 638615 \\
\hline 59 & 1 & 0 & -2.981434 & -3.620753 & 0.810585 \\
\hline 60 & 6 & 0 & -1.495156 & -4.251488 & 3. 826016 \\
\hline 61 & 1 & 0 & -0.417819 & -2.785996 & 5. 006929 \\
\hline 62 & 1 & 0 & -2.616280 & -5.464302 & 2. 421765 \\
\hline
\end{tabular}




\begin{tabular}{|c|c|c|c|c|c|}
\hline 63 & 1 & 0 & -1.335000 & -5.062743 & 4. 531175 \\
\hline 64 & 46 & 0 & -0.244161 & -0.145965 & -0.669818 \\
\hline 65 & 6 & 0 & 0.569622 & -2.182588 & -1.304029 \\
\hline 66 & 6 & 0 & 1. 079596 & -1.167537 & -2.167181 \\
\hline 67 & 6 & 0 & 1. 774768 & -0.054136 & -1.628095 \\
\hline 68 & 1 & 0 & 0.788583 & -1.148779 & -3.216990 \\
\hline 69 & 1 & 0 & 2. 249478 & -0.178394 & -0.654719 \\
\hline 70 & 1 & 0 & 1. 114081 & -2.360913 & -0.373930 \\
\hline 71 & 6 & 0 & -0.265233 & -3.313142 & -1.761039 \\
\hline 72 & 6 & 0 & -1.026575 & -3.274420 & -2.957639 \\
\hline 73 & 6 & 0 & -0.284804 & -4.500012 & -0.987669 \\
\hline 74 & 6 & 0 & -1.766364 & -4.391323 & -3.372442 \\
\hline 75 & 1 & 0 & -1.047459 & -2.372055 & -3.565473 \\
\hline 76 & 6 & 0 & -1.024688 & -5.619801 & -1.404050 \\
\hline 77 & 1 & 0 & 0.295604 & -4.547904 & -0.068704 \\
\hline 78 & 6 & 0 & -1.767927 & -5.571420 & -2.598961 \\
\hline 79 & 1 & 0 & -2.341764 & -4.347723 & -4.293771 \\
\hline 80 & 1 & 0 & -1.014878 & -6.526602 & -0.804935 \\
\hline 81 & 1 & 0 & -2.338216 & -6.437257 & -2.925047 \\
\hline 82 & 6 & 0 & 2. 343103 & 1. 061121 & -2.482537 \\
\hline 83 & 1 & 0 & 2. 405054 & 1. 981052 & -1.886875 \\
\hline 84 & 1 & 0 & 1. 671602 & 1. 269570 & -3.326705 \\
\hline 85 & 6 & 0 & 3. 757561 & 0.756525 & -3.060563 \\
\hline 86 & 1 & 0 & 3. 715183 & -0.135368 & -3.703273 \\
\hline 87 & 1 & 0 & 4. 037158 & 1. 595020 & -3.714861 \\
\hline 88 & 6 & 0 & 4. 904613 & 0.593738 & -2.033369 \\
\hline 89 & 1 & 0 & 4. 809679 & 1. 332927 & -1.229262 \\
\hline 90 & 1 & 0 & 5. 868461 & 0.766516 & -2.527970 \\
\hline 91 & 7 & 0 & 4. 964226 & -0.749440 & -1.411254 \\
\hline 92 & 1 & 0 & 4. 983231 & -1.597045 & -1.973999 \\
\hline 93 & 16 & 0 & 4. 662228 & -1.046097 & 0.263568 \\
\hline 94 & 8 & 0 & 4. 046281 & 0.296538 & 0.919403 \\
\hline 95 & 8 & 0 & 3. 943521 & -2.479588 & 0.420877 \\
\hline 96 & 6 & 0 & 6. 533937 & -1.230165 & 1. 049184 \\
\hline 97 & 9 & 0 & 7. 213997 & -0.055618 & 0.805191 \\
\hline 98 & 9 & 0 & 6. 450987 & -1.460810 & 2. 397721 \\
\hline 99 & 9 & 0 & 7. 165440 & -2.278595 & 0.418749 \\
\hline
\end{tabular}




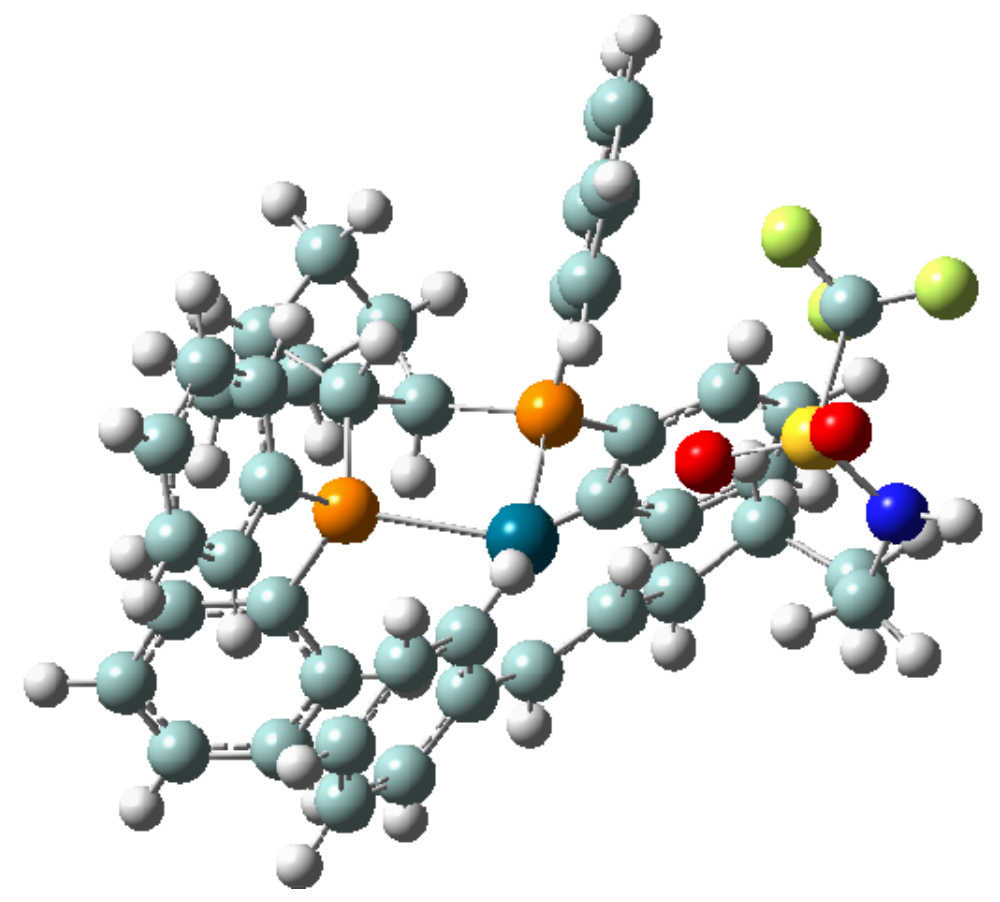

$\pi$-Allyl intermediate in hydroamination, isomer II

$\mathrm{E}(\mathrm{RB}+\mathrm{HF}-\mathrm{LYP})=-3416.61177187 \quad$ A. $\mathrm{U}$.

Standard orientation:

\begin{tabular}{|c|c|c|c|c|c|}
\hline \multirow{2}{*}{$\begin{array}{l}\text { Center } \\
\text { Number }\end{array}$} & \multirow{2}{*}{$\begin{array}{l}\text { Atomic } \\
\text { Number }\end{array}$} & \multirow{2}{*}{$\begin{array}{l}\text { Atomic } \\
\text { Type }\end{array}$} & \multicolumn{3}{|c|}{ Coordinates (Angstroms) } \\
\hline & & & $\mathrm{X}$ & Y & Z \\
\hline 1 & 6 & 0 & 4. 754269 & -2.361009 & 1. 488788 \\
\hline 2 & 6 & 0 & 3.555586 & -1.783976 & 2. 304409 \\
\hline 3 & 6 & 0 & 2. 664478 & -3.607229 & 1. 235918 \\
\hline 4 & 6 & 0 & 4. 170606 & -3.672951 & 0.840829 \\
\hline 5 & 1 & 0 & 5. 130238 & -1.660438 & 0.739322 \\
\hline 6 & 1 & 0 & 5.585669 & -2.591720 & 2. 164290 \\
\hline 7 & 1 & 0 & 4. 321060 & -3.710387 & -0.244618 \\
\hline 8 & 1 & 0 & 4. 637107 & -4.567793 & 1. 269230 \\
\hline
\end{tabular}




\begin{tabular}{|c|c|c|c|c|c|}
\hline 9 & 6 & 0 & 2. 791676 & -3.091329 & 2. 700602 \\
\hline 10 & 1 & 0 & 1. 835850 & -2.905817 & 3. 201580 \\
\hline 11 & 1 & 0 & 3. 390791 & -3.764002 & 3. 323979 \\
\hline 12 & 6 & 0 & 2. 449715 & -1.173669 & 1. 393316 \\
\hline 13 & 1 & 0 & 1. 589529 & -0.993219 & 2. 055661 \\
\hline 14 & 6 & 0 & 2. 090442 & -2.373190 & 0.457348 \\
\hline 15 & 1 & 0 & 2. 635510 & -2.280312 & -0.491203 \\
\hline 16 & 1 & 0 & 3. 850744 & -1.132253 & 3. 130787 \\
\hline 17 & 1 & 0 & 2. 122103 & -4.543899 & 1. 086496 \\
\hline 18 & 15 & 0 & 0.282081 & -2.158336 & -0.086850 \\
\hline 19 & 15 & 0 & 2. 462077 & 0.440675 & 0.389944 \\
\hline 20 & 6 & 0 & 2. 644927 & 1. 814881 & 1. 640258 \\
\hline 21 & 6 & 0 & 3. 101009 & 3. 082341 & 1. 215839 \\
\hline 22 & 6 & 0 & 2. 272943 & 1. 631545 & 2. 990552 \\
\hline 23 & 6 & 0 & 3. 201372 & 4. 142959 & 2. 132743 \\
\hline 24 & 1 & 0 & 3. 379329 & 3. 249466 & 0.179173 \\
\hline 25 & 6 & 0 & 2. 373472 & 2. 695706 & 3. 905206 \\
\hline 26 & 1 & 0 & 1. 907856 & 0.673070 & 3. 347951 \\
\hline 27 & 6 & 0 & 2. 840771 & 3. 953961 & 3. 480390 \\
\hline 28 & 1 & 0 & 3. 554139 & 5. 112266 & 1. 791348 \\
\hline 29 & 1 & 0 & 2. 089695 & 2. 540354 & 4. 942806 \\
\hline 30 & 1 & 0 & 2. 920291 & 4. 774563 & 4. 188496 \\
\hline 31 & 6 & 0 & 3. 996188 & 0.509124 & -0.676252 \\
\hline 32 & 6 & 0 & 3. 887954 & 0.152577 & -2.037703 \\
\hline 33 & 6 & 0 & 5.251940 & 0.893130 & -0.156542 \\
\hline 34 & 6 & 0 & 5. 023674 & 0.164658 & -2.869113 \\
\hline 35 & 1 & 0 & 2. 919170 & -0.121628 & -2.450728 \\
\hline 36 & 6 & 0 & 6. 384407 & 0.912807 & -0.990127 \\
\hline 37 & 1 & 0 & 5. 349253 & 1. 180964 & 0.886641 \\
\hline 38 & 6 & 0 & 6. 274235 & 0.545047 & -2.346594 \\
\hline 39 & 1 & 0 & 4. 931212 & -0.112096 & -3.916221 \\
\hline 40 & 1 & 0 & 7. 346974 & 1. 211696 & -0.583702 \\
\hline 41 & 1 & 0 & 7. 151546 & 0.561230 & -2.987883 \\
\hline 42 & 6 & 0 & -0.791998 & -2.456833 & 1. 408802 \\
\hline 43 & 6 & 0 & -1.503389 & -1.369046 & 1. 958931 \\
\hline 44 & 6 & 0 & -0.881363 & -3.729330 & 2. 017709 \\
\hline 45 & 6 & 0 & -2.293511 & -1.548115 & 3. 110319 \\
\hline 46 & 1 & 0 & -1.451655 & -0.390226 & 1. 487182 \\
\hline 47 & 6 & 0 & -1.670906 & -3.905571 & 3. 167566 \\
\hline 48 & 1 & 0 & -0.350628 & -4.579980 & 1. 598290 \\
\hline 49 & 6 & 0 & -2.376016 & -2.815061 & 3. 717589 \\
\hline 50 & 1 & 0 & -2.840021 & -0.705775 & 3. 525613 \\
\hline 51 & 1 & 0 & -1.736593 & -4.886331 & 3. 631088 \\
\hline 52 & 1 & 0 & -2.986651 & -2.955270 & 4. 605705 \\
\hline 53 & 6 & 0 & -0.078597 & -3.542637 & -1.286283 \\
\hline 54 & 6 & 0 & 0.897257 & -3.922808 & -2.235180 \\
\hline 55 & 6 & 0 & -1.343883 & -4.169860 & -1.308469 \\
\hline 56 & 6 & 0 & 0.616808 & -4.925673 & -3.180258 \\
\hline 57 & 1 & 0 & 1. 876754 & -3.450677 & -2.247836 \\
\hline 58 & 6 & 0 & -1.621884 & -5.172346 & -2.256532 \\
\hline 59 & 1 & 0 & -2.107352 & -3.894217 & -0.585860 \\
\hline 60 & 6 & 0 & -0.643791 & -5.554410 & -3.194139 \\
\hline
\end{tabular}




\begin{tabular}{rrrrrr}
61 & 1 & 0 & 1.378422 & -5.215140 & -3.899548 \\
62 & 1 & 0 & -2.596344 & -5.653749 & -2.258447 \\
63 & 1 & 0 & -0.858836 & -6.331122 & -3.923052 \\
64 & 46 & 0 & 0.346541 & 0.159633 & -0.837791 \\
65 & 6 & 0 & -1.446788 & 0.230099 & -2.163170 \\
66 & 6 & 0 & -1.175515 & 1.545547 & -1.683916 \\
67 & 6 & 0 & 0.077955 & 2.162917 & -1.948553 \\
68 & 1 & 0 & -1.857038 & 1.982163 & -0.956875 \\
69 & 1 & 0 & 0.625235 & 1.828142 & -2.834019 \\
70 & 1 & 0 & -0.935125 & -0.086562 & -3.078845 \\
71 & 6 & 0 & -4.534519 & 1.291241 & -2.590890 \\
72 & 1 & 0 & -5.075816 & 1.613306 & -3.489255 \\
73 & 1 & 0 & -3.767245 & 2.048883 & -2.392642 \\
74 & 7 & 0 & -5.563066 & 1.294092 & -1.505191 \\
75 & 1 & 0 & -6.511840 & 1.582324 & -1.750120 \\
76 & 6 & 0 & 0.473966 & 3.496120 & -1.451882 \\
77 & 6 & 0 & 1.532177 & 4.176408 & -2.104765 \\
78 & 6 & 0 & -0.193035 & 4.149477 & -0.383661 \\
79 & 6 & 0 & 1.902495 & 5.475921 & -1.720107 \\
80 & 1 & 0 & 2.051124 & 3.691656 & -2.930363 \\
81 & 6 & 0 & 0.175938 & 5.447491 & -0.001320 \\
82 & 1 & 0 & -1.000600 & 3.652883 & 0.148737 \\
83 & 6 & 0 & 1.221478 & 6.119472 & -0.668228 \\
84 & 1 & 0 & 2.705842 & 5.987619 & -2.243845 \\
85 & 1 & 0 & -0.350336 & 5.937443 & 0.813652 \\
86 & 1 & 0 & 1.496117 & 7.129613 & -0.375551 \\
87 & 6 & 0 & -2.748587 & -0.501892 & -1.877459 \\
88 & 1 & 0 & -2.582737 & -1.578606 & -2.003963 \\
89 & 1 & 0 & -3.055954 & -0.342405 & -0.839555 \\
90 & 6 & 0 & -3.901919 & -0.092816 & -2.841612 \\
91 & 1 & 0 & -3.523672 & -0.103168 & -3.875218 \\
92 & 1 & 0 & -4.697849 & -0.846394 & -2.788062 \\
93 & 16 & 0 & -5.179118 & 1.856659 & 0.121619 \\
94 & 8 & 0 & -3.573339 & 1.945846 & 0.259664 \\
95 & 8 & 0 & -6.161917 & 3.037635 & 0.590690 \\
96 & 6 & 0 & -5.731539 & 0.222279 & 1.192597 \\
97 & 9 & -5.468578 & 0.437400 & 2.525466 \\
98 & 9 & -7.076903 & 0.008343 & 1.000783 \\
99 & 9 & -5.015867 & -0.872568 & 0.750112 \\
------------------------------ \\
\hline 5
\end{tabular}




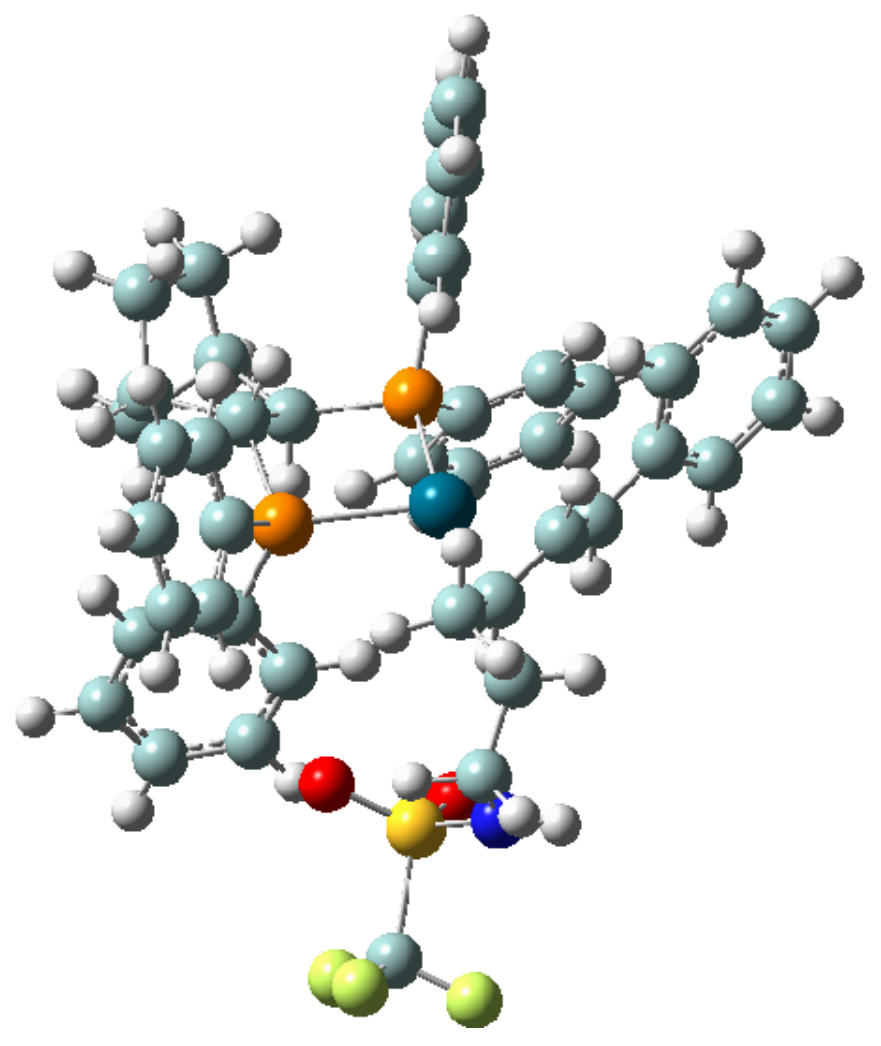

\section{$\pi$-Allyl intermediate in hydroamination, isomer III}

$\mathrm{E}(\mathrm{RB}+\mathrm{HF}-\mathrm{LYP})=-3416.61386442 \quad$ A. U.

Standard orientation:

\begin{tabular}{rrrrrr} 
Center & Atomic & Atomic & \multicolumn{3}{c}{ Coordinates (Angstroms) } \\
Number & Number & Type & X & Y & $Z$ \\
----1 & 6 & 0 & -3.607910 & 3.866803 & 0.633044 \\
1 & 6 & 0 & -2.718351 & 3.166879 & 1.707188 \\
2 & 6 & 0 & -1.203266 & 4.187143 & 0.320191 \\
3 & 6 & 0 & -2.573775 & 4.658554 & -0.253062 \\
4 & 6 & 0 & -4.198054 & 3.156984 & 0.048358 \\
5 & 1 & 0 & -4.311566 & 4.549649 & 1.122274 \\
6 & 1 & 0 & -2.683589 & 4.441526 & -1.322494 \\
7 & 1 & 0 & -2.688143 & 5.741117 & -0.122871 \\
8 & 1 & 0 & -1.535955 & 4.183229 & 1.842496 \\
9 & 6 & 0 & -0.722417 & 3.828560 & 2.483550 \\
10 & 1 & 0 & -1.857729 & 5.169126 & 2.195843 \\
11 & 1 & 0 & -1.893291 & 1.981713 & 1.123572 \\
12 & 6 & 0 & -1.168690 & 1.715090 & 1.907661 \\
13 & 1 & & & &
\end{tabular}




\begin{tabular}{|c|c|c|c|c|c|}
\hline 14 & 6 & 0 & -1.109357 & 2. 664303 & -0.044430 \\
\hline 15 & 1 & 0 & -1.641235 & 2. 503149 & -0.991490 \\
\hline 16 & 1 & 0 & -3.245798 & 2. 920009 & 2. 632364 \\
\hline 17 & 1 & 0 & -0.352038 & 4. 793995 & 0.003288 \\
\hline 18 & 15 & 0 & 0.508796 & 1. 698384 & -0.296863 \\
\hline 19 & 15 & 0 & -2.477797 & 0.263095 & 0.568009 \\
\hline 20 & 6 & 0 & -3.157036 & -0.577091 & 2. 089645 \\
\hline 21 & 6 & 0 & -4.022596 & -1.684438 & 1. 942760 \\
\hline 22 & 6 & 0 & -2.766784 & -0.172734 & 3. 385534 \\
\hline 23 & 6 & 0 & -4.502872 & -2.363414 & 3. 076698 \\
\hline 24 & 1 & 0 & -4.328993 & -2.017991 & 0.955359 \\
\hline 25 & 6 & 0 & -3.246872 & -0.856715 & 4. 517429 \\
\hline 26 & 1 & 0 & -2.097578 & 0.670025 & 3. 532173 \\
\hline 27 & 6 & 0 & -4.117916 & -1.952593 & 4. 367436 \\
\hline 28 & 1 & 0 & -5.173466 & -3.209170 & 2. 949801 \\
\hline 29 & 1 & 0 & -2.943848 & -0.532144 & 5. 509415 \\
\hline 30 & 1 & 0 & -4.491232 & -2.477428 & 5. 242799 \\
\hline 31 & 6 & 0 & -3.919248 & 0.459400 & -0.605569 \\
\hline 32 & 6 & 0 & -3.661504 & 0.456166 & -1.993157 \\
\hline 33 & 6 & 0 & -5.241443 & 0.640263 & -0.144217 \\
\hline 34 & 6 & 0 & -4.710509 & 0.646912 & -2.911620 \\
\hline 35 & 1 & 0 & -2.647449 & 0.296546 & -2.354543 \\
\hline 36 & 6 & 0 & -6.290543 & 0.819361 & -1.063650 \\
\hline 37 & 1 & 0 & -5.456999 & 0.640824 & 0.920686 \\
\hline 38 & 6 & 0 & -6.027540 & 0.828014 & -2.448443 \\
\hline 39 & 1 & 0 & -4.502823 & 0.646752 & -3.978700 \\
\hline 40 & 1 & 0 & -7.306158 & 0.955566 & -0.701596 \\
\hline 41 & 1 & 0 & -6.840414 & 0.969804 & -3.155846 \\
\hline 42 & 6 & 0 & 1. 545280 & 2. 008913 & 1. 224411 \\
\hline 43 & 6 & 0 & 1. 730186 & 0.968829 & 2. 158137 \\
\hline 44 & 6 & 0 & 2. 114974 & 3. 278681 & 1. 469435 \\
\hline 45 & 6 & 0 & 2. 470904 & 1. 196347 & 3. 333376 \\
\hline 46 & 1 & 0 & 1. 330599 & -0.023048 & 1. 963646 \\
\hline 47 & 6 & 0 & 2. 854122 & 3.503257 & 2. 643455 \\
\hline 48 & 1 & 0 & 2. 000430 & 4. 083480 & 0.748171 \\
\hline 49 & 6 & 0 & 3. 030220 & 2. 463718 & 3. 580007 \\
\hline 50 & 1 & 0 & 2. 619380 & 0.384232 & 4. 039591 \\
\hline 51 & 1 & 0 & 3. 293444 & 4. 480647 & 2. 824869 \\
\hline 52 & 1 & 0 & 3. 607022 & 2. 639054 & 4. 484469 \\
\hline 53 & 6 & 0 & 1. 412459 & 2. 527472 & -1.702733 \\
\hline 54 & 6 & 0 & 0.704146 & 3. 199389 & -2.723180 \\
\hline 55 & 6 & 0 & 2. 821009 & 2. 437003 & -1.775002 \\
\hline 56 & 6 & 0 & 1. 397224 & 3. 787341 & -3.797276 \\
\hline 57 & 1 & 0 & -0.379127 & 3. 282820 & -2.690043 \\
\hline 58 & 6 & 0 & 3. 508983 & 3. 029823 & -2.849808 \\
\hline 59 & 1 & 0 & 3. 381862 & 1. 915669 & -1.003438 \\
\hline 60 & 6 & 0 & 2. 801734 & 3. 705951 & -3.862998 \\
\hline 61 & 1 & 0 & 0.843788 & 4. 310494 & -4.572807 \\
\hline 62 & 1 & 0 & 4. 593189 & 2. 965036 & -2.890934 \\
\hline 63 & 1 & 0 & 3. 336684 & 4. 166175 & -4.689634 \\
\hline 64 & 46 & 0 & -0.419126 & -0.559325 & -0.496602 \\
\hline 65 & 6 & 0 & -0.798445 & -2.827931 & -0.638501 \\
\hline
\end{tabular}




\begin{tabular}{|c|c|c|c|c|c|}
\hline 66 & 6 & 0 & 0.052381 & -2.395557 & -1.691622 \\
\hline 67 & 6 & 0 & 1. 267556 & -1.704437 & -1.418301 \\
\hline 68 & 1 & 0 & -0.304612 & -2.440137 & -2.720120 \\
\hline 69 & 1 & 0 & 1. 759876 & -1.916690 & -0.466540 \\
\hline 70 & 1 & 0 & -0.332734 & -3.020267 & 0. 331692 \\
\hline 71 & 6 & 0 & -2.099946 & -3.496580 & -0.842596 \\
\hline 72 & 6 & 0 & -2.880287 & -3.321133 & -2.014002 \\
\hline 73 & 6 & 0 & -2.570408 & -4.385704 & 0.155106 \\
\hline 74 & 6 & 0 & -4.079361 & -4.027290 & -2.187099 \\
\hline 75 & 1 & 0 & -2.560033 & -2.625816 & -2.786468 \\
\hline 76 & 6 & 0 & -3.769751 & -5.097046 & -0.019954 \\
\hline 77 & 1 & 0 & -1.985997 & -4.529799 & 1. 061769 \\
\hline 78 & 6 & 0 & -4.528841 & -4.922404 & -1.193112 \\
\hline 79 & 1 & 0 & -4.666273 & -3.882515 & -3.090353 \\
\hline 80 & 1 & 0 & -4.105917 & -5.787419 & 0.749244 \\
\hline 81 & 1 & 0 & -5.455209 & -5.473223 & -1.332890 \\
\hline 82 & 6 & 0 & 2. 140920 & -1.195063 & -2.554167 \\
\hline 83 & 1 & 0 & 2. 652136 & -0.273176 & -2.257691 \\
\hline 84 & 1 & 0 & 1. 495135 & -0.935210 & -3.403631 \\
\hline 85 & 6 & 0 & 3. 205134 & -2.224242 & -3.052122 \\
\hline 86 & 1 & 0 & 2. 791610 & -3.242917 & -3.020952 \\
\hline 87 & 1 & 0 & 3. 421383 & -2.011913 & -4.109506 \\
\hline 88 & 6 & 0 & 4. 569687 & -2.188728 & -2.329099 \\
\hline 89 & 1 & 0 & 4. 989797 & -1.177475 & -2.365712 \\
\hline 90 & 1 & 0 & 5. 279021 & -2.856592 & -2.834154 \\
\hline 91 & 7 & 0 & 4. 545098 & -2.610758 & -0.908680 \\
\hline 92 & 1 & 0 & 4. 227776 & -3.541014 & -0.641678 \\
\hline 93 & 16 & 0 & 4. 448562 & -1.461521 & 0.389644 \\
\hline 94 & 8 & 0 & 3. 340616 & -1.953480 & 1. 457516 \\
\hline 95 & 8 & 0 & 4. 445186 & 0.021440 & -0.241131 \\
\hline 96 & 6 & 0 & 6. 250213 & -1.693479 & 1. 304745 \\
\hline 97 & 9 & 0 & 6. 282046 & -0.941122 & 2. 450944 \\
\hline 98 & 9 & 0 & 6. 406856 & -3.029240 & 1. 605225 \\
\hline 99 & 9 & 0 & 7. 233404 & -1.285524 & 0.434415 \\
\hline
\end{tabular}




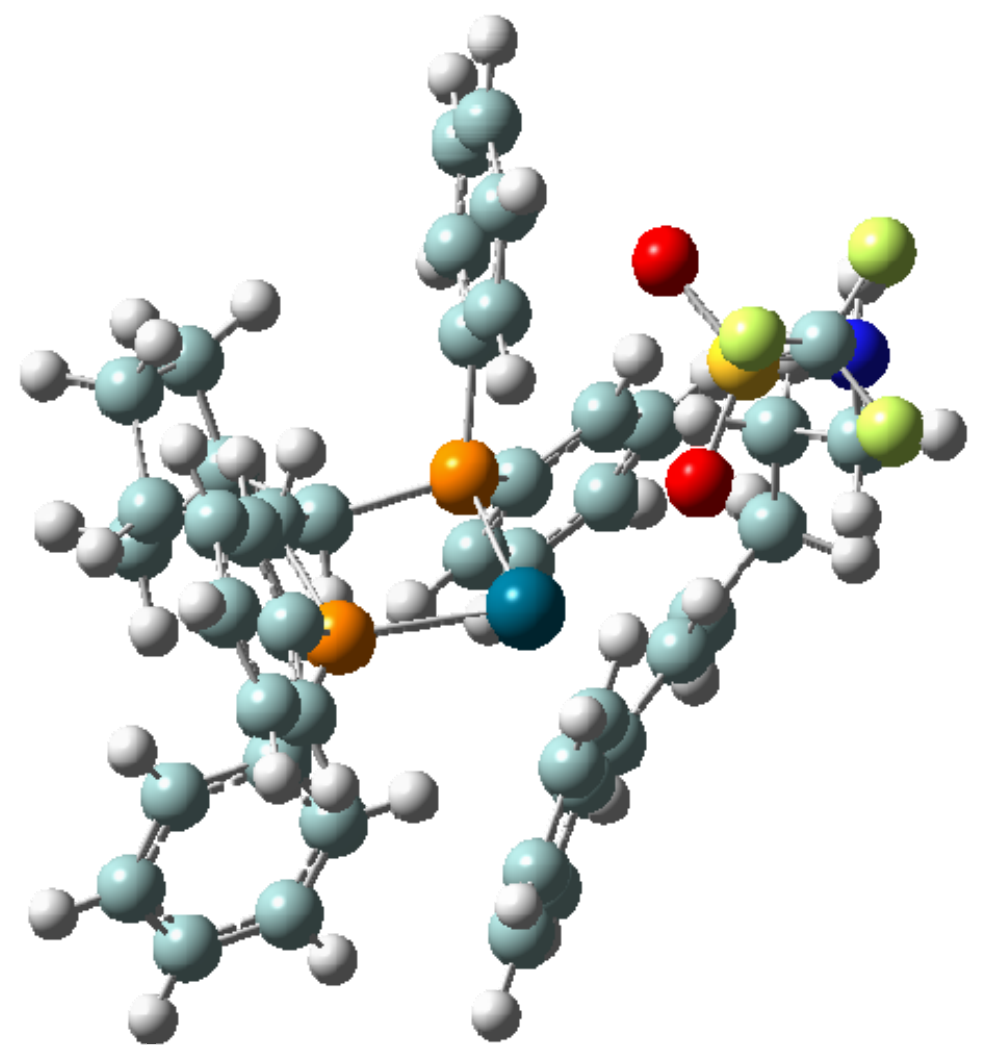

$\pi$-Allyl intermediate in hydroamination, isomer IV

\begin{tabular}{|c|c|c|c|c|c|}
\hline \multirow{2}{*}{$\begin{array}{l}\text { Center } \\
\text { Number }\end{array}$} & \multirow{2}{*}{$\begin{array}{l}\text { Atomic } \\
\text { Number }\end{array}$} & \multirow{2}{*}{$\begin{array}{l}\text { Atomic } \\
\text { Type }\end{array}$} & \multicolumn{3}{|c|}{ Coordinates (Angstroms) } \\
\hline & & & X & Y & Z \\
\hline 1 & 6 & 0 & 3. 700219 & -2.073627 & 3. 022215 \\
\hline 2 & 6 & 0 & 4. 167832 & -1.649591 & 1. 595574 \\
\hline 3 & 6 & 0 & 3. 658517 & 0.339012 & 2. 619232 \\
\hline 4 & 6 & 0 & 3. 451205 & -0.703848 & 3. 758891 \\
\hline 5 & 1 & 0 & 2. 806239 & -2.701333 & 3. 003875 \\
\hline 6 & 1 & 0 & 4. 491549 & -2.648651 & 3. 516234 \\
\hline 7 & 1 & 0 & 2. 454157 & -0.645720 & 4. 211711 \\
\hline 8 & 1 & 0 & 4. 185362 & -0.544879 & 4. 557364 \\
\hline 9 & 6 & 0 & 4. 885477 & -0.291158 & 1. 895238 \\
\hline 10 & 1 & 0 & 5. 204509 & 0.245879 & 0.995663 \\
\hline 11 & 1 & 0 & 5. 746781 & -0.402088 & 2. 562974 \\
\hline 12 & 6 & 0 & 3. 009893 & -1.071753 & 0.730177 \\
\hline
\end{tabular}




\begin{tabular}{|c|c|c|c|c|c|}
\hline 13 & 1 & 0 & 3. 493253 & -0.639643 & -0.159280 \\
\hline 14 & 6 & 0 & 2. 477001 & 0.099508 & 1. 616273 \\
\hline 15 & 1 & 0 & 1. 601033 & -0.242286 & 2. 182810 \\
\hline 16 & 1 & 0 & 4. 766212 & -2.403849 & 1. 077604 \\
\hline 17 & 1 & 0 & 3. 771051 & 1. 368282 & 2. 967147 \\
\hline 18 & 15 & 0 & 1. 745946 & 1. 426367 & 0.470334 \\
\hline 19 & 15 & 0 & 1. 527753 & -1.955062 & -0.066569 \\
\hline 20 & 6 & 0 & 2. 233897 & -3.267359 & -1.192076 \\
\hline 21 & 6 & 0 & 1. 479625 & -4.420760 & -1.504575 \\
\hline 22 & 6 & 0 & 3. 492876 & -3.083065 & -1.805999 \\
\hline 23 & 6 & 0 & 1. 981606 & -5.377681 & -2.405323 \\
\hline 24 & 1 & 0 & 0.513869 & -4.588519 & -1.034690 \\
\hline 25 & 6 & 0 & 3. 992106 & -4.040597 & -2.707550 \\
\hline 26 & 1 & 0 & 4. 096755 & -2.205658 & -1.590475 \\
\hline 27 & 6 & 0 & 3. 239096 & -5.191127 & -3.010802 \\
\hline 28 & 1 & 0 & 1. 395268 & -6.265173 & -2.628897 \\
\hline 29 & 1 & 0 & 4. 965282 & -3.889446 & -3.167218 \\
\hline 30 & 1 & 0 & 3. 627491 & -5.931653 & -3.704708 \\
\hline 31 & 6 & 0 & 3. 196535 & 2. 307166 & -0.315258 \\
\hline 32 & 6 & 0 & 3. 577558 & 1. 960985 & -1.629795 \\
\hline 33 & 6 & 0 & 3. 940809 & 3. 277680 & 0.390838 \\
\hline 34 & 6 & 0 & 4. 694851 & 2. 570177 & -2.230821 \\
\hline 35 & 1 & 0 & 2. 998866 & 1. 226849 & -2.187165 \\
\hline 36 & 6 & 0 & 5. 054447 & 3. 889166 & -0.212700 \\
\hline 37 & 1 & 0 & 3. 650022 & 3. 568434 & 1. 396607 \\
\hline 38 & 6 & 0 & 5. 435998 & 3.535166 & -1.522516 \\
\hline 39 & 1 & 0 & 4. 979411 & 2. 299737 & -3.244498 \\
\hline 40 & 1 & 0 & 5. 620161 & 4. 638024 & 0.335266 \\
\hline 41 & 1 & 0 & 6. 295941 & 4. 011162 & -1.986344 \\
\hline 42 & 6 & 0 & 0.944346 & 2. 682395 & 1. 593681 \\
\hline 43 & 6 & 0 & 0.597053 & 2. 362758 & 2. 924720 \\
\hline 44 & 6 & 0 & 0.634680 & 3. 963596 & 1. 086156 \\
\hline 45 & 6 & 0 & -0.038744 & 3. 316646 & 3. 740415 \\
\hline 46 & 1 & 0 & 0.819116 & 1. 386042 & 3. 343969 \\
\hline 47 & 6 & 0 & 0.002150 & 4. 914881 & 1. 905056 \\
\hline 48 & 1 & 0 & 0.882521 & 4. 226427 & 0.061651 \\
\hline 49 & 6 & 0 & -0.336182 & 4. 596012 & 3. 234190 \\
\hline 50 & 1 & 0 & -0.296717 & 3. 060895 & 4. 764765 \\
\hline 51 & 1 & 0 & -0.229565 & 5. 896676 & 1.501586 \\
\hline 52 & 1 & 0 & -0.825365 & 5. 332762 & 3.865925 \\
\hline 53 & 46 & 0 & 0.435180 & 0.009252 & -1.059650 \\
\hline 54 & 6 & 0 & -0.768912 & -0.884196 & -2.738401 \\
\hline 55 & 6 & 0 & -1.341579 & 0.363291 & -2.363817 \\
\hline 56 & 6 & 0 & -0.563019 & 1. 552304 & -2.446538 \\
\hline 57 & 1 & 0 & -2.295982 & 0.394726 & -1.844825 \\
\hline 58 & 1 & 0 & 0.265831 & 1. 553176 & -3.159447 \\
\hline 59 & 1 & 0 & 0.043504 & -0.853525 & -3.471818 \\
\hline 60 & 6 & 0 & -1.494078 & -2.222900 & -2.704444 \\
\hline 61 & 1 & 0 & -1.979906 & -2.373089 & -3.684896 \\
\hline 62 & 1 & 0 & -0.735445 & -3.011987 & -2.639785 \\
\hline 63 & 6 & 0 & -2.535243 & -2.424423 & -1.575323 \\
\hline 64 & 1 & 0 & -2.588111 & -3.492238 & -1.320323 \\
\hline
\end{tabular}




\begin{tabular}{|c|c|c|c|c|c|}
\hline 65 & 1 & 0 & -2.189343 & -1.905367 & -0.673658 \\
\hline 66 & 6 & 0 & -3.960344 & -1.942494 & -1.932904 \\
\hline 67 & 1 & 0 & -3.961372 & -0.925683 & -2.333908 \\
\hline 68 & 1 & 0 & -4.417352 & -2.587549 & -2.694719 \\
\hline 69 & 7 & 0 & -4.915449 & -1.975041 & -0.790301 \\
\hline 70 & 1 & 0 & -5.055823 & -2.881606 & -0.332672 \\
\hline 71 & 6 & 0 & -1.036015 & 2. 896564 & -2.061723 \\
\hline 72 & 6 & 0 & -2.162145 & 3. 108326 & -1.224966 \\
\hline 73 & 6 & 0 & -0.364358 & 4. 024956 & -2.596670 \\
\hline 74 & 6 & 0 & -2.609575 & 4. 410827 & -0.957576 \\
\hline 75 & 1 & 0 & -2.691093 & 2. 268332 & -0.782009 \\
\hline 76 & 6 & 0 & -0.812222 & 5. 328125 & -2.324189 \\
\hline 77 & 1 & 0 & 0.498653 & 3. 877648 & -3.244198 \\
\hline 78 & 6 & 0 & -1.942146 & 5.525797 & -1.505809 \\
\hline 79 & 1 & 0 & -3.478204 & 4. 558563 & -0.321337 \\
\hline 80 & 1 & 0 & -0.294413 & 6. 180680 & -2.755875 \\
\hline 81 & 1 & 0 & -2.300573 & 6. 531792 & -1.302749 \\
\hline 82 & 6 & 0 & 0.540440 & -2.850724 & 1. 243551 \\
\hline 83 & 6 & 0 & -0.631699 & -2.233646 & 1. 732348 \\
\hline 84 & 6 & 0 & 0.931770 & -4.106582 & 1. 759605 \\
\hline 85 & 6 & 0 & -1.409584 & -2.859817 & 2. 724848 \\
\hline 86 & 1 & 0 & -0.947368 & -1.271334 & 1. 334837 \\
\hline 87 & 6 & 0 & 0.156448 & -4.730746 & 2. 753323 \\
\hline 88 & 1 & 0 & 1. 827782 & -4.599619 & 1. 392175 \\
\hline 89 & 6 & 0 & -1.013032 & -4.109177 & 3. 238285 \\
\hline 90 & 1 & 0 & -2.319852 & -2.378574 & 3. 070430 \\
\hline 91 & 1 & 0 & 0.463643 & -5.695925 & 3. 147580 \\
\hline 92 & 1 & 0 & -1.610519 & -4.598125 & 4. 003360 \\
\hline 93 & 16 & 0 & -4.686224 & -0.724095 & 0.467313 \\
\hline 94 & 8 & 0 & -3.819948 & 0.477270 & -0.178753 \\
\hline 95 & 8 & 0 & -4.312460 & -1.356779 & 1.901416 \\
\hline 96 & 6 & 0 & -6.592081 & -0.052689 & 0.594602 \\
\hline 97 & 9 & 0 & -6.936015 & 0.516312 & -0.606218 \\
\hline 98 & 9 & 0 & -7.407137 & -1.126410 & 0.870074 \\
\hline 99 & 9 & 0 & -6.669838 & 0.875085 & 1. 602424 \\
\hline
\end{tabular}




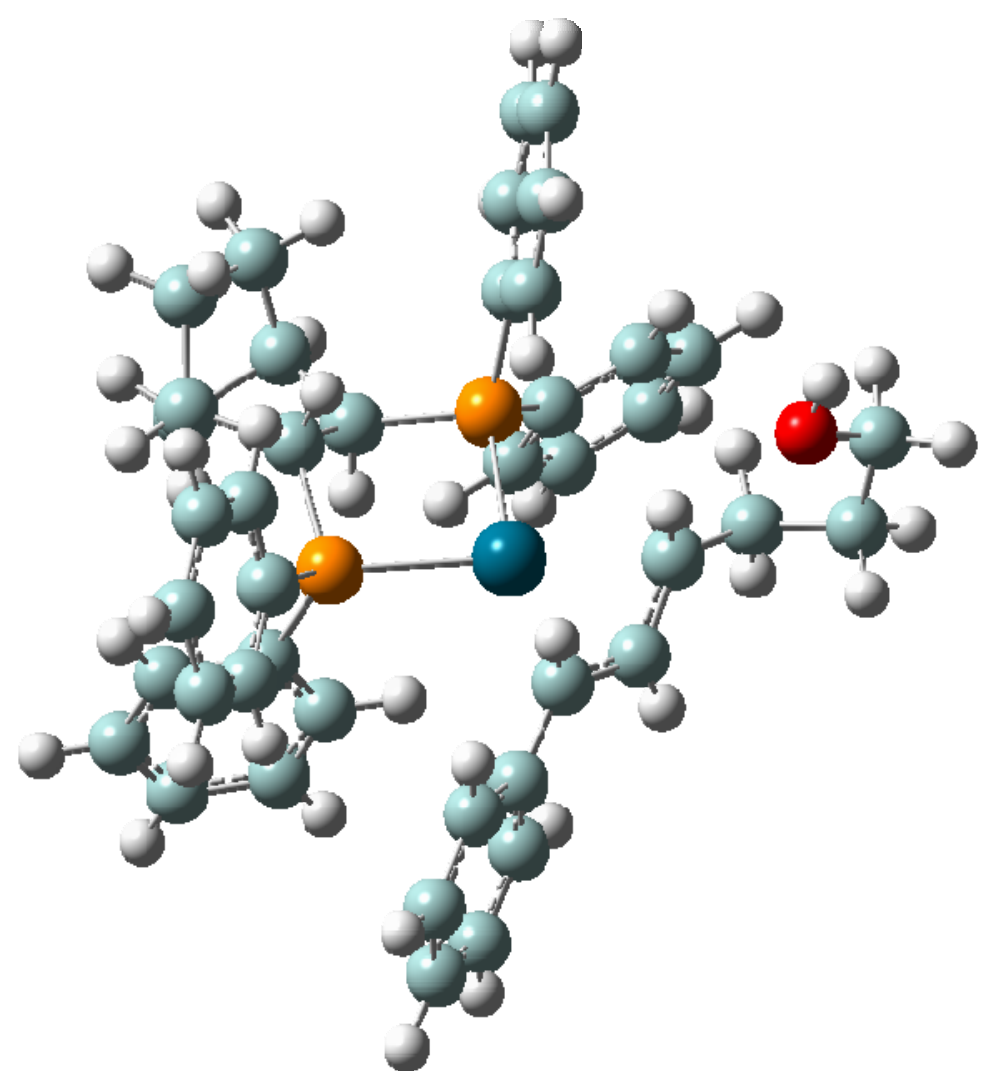

\section{$\pi$-Allyl intermediate in hydroalkoxylation, isomer I}

$\mathrm{E}(\mathrm{RB}+\mathrm{HF}-\mathrm{LYP})=-2551.06544537 \quad$ A. U.

Standard orientation:

\begin{tabular}{rrrrrr} 
Center & Atomic & Atomic & \multicolumn{3}{c}{ Coordinates (Angstroms) } \\
Number & Number & Type & X & Y \\
-1 & 6 & 0 & 1.870321 & -4.277842 & -0.544978 \\
2 & 6 & 0 & 1.366085 & -3.634345 & 0.783957 \\
3 & 6 & 0 & -0.536259 & -3.844240 & -0.480939 \\
4 & 6 & 0 & 0.551464 & -4.513797 & -1.373224 \\
5 & 1 & 0 & 2.583444 & -3.644828 & -1.077513 \\
6 & 1 & 0 & 2.374808 & -5.226368 & -0.328683 \\
7 & 1 & 0 & 0.602398 & -4.077082 & -2.377862 \\
8 & 1 & 0 & 0.342520 & -5.583723 & -1.489565 \\
9 & 6 & 0 & -0.046372 & -4.293952 & 0.927971 \\
10 & 1 & 0 & -0.635257 & -3.895024 & 1.760322 \\
11 & 1 & 0 & -0.000742 & -5.384678 & 1.020383
\end{tabular}




\begin{tabular}{|c|c|c|c|c|c|}
\hline 12 & 6 & 0 & 0.879894 & -2.166831 & 0.597497 \\
\hline 13 & 1 & 0 & 0.375118 & -1.907089 & 1. 540113 \\
\hline 14 & 6 & 0 & -0.212647 & -2.310709 & -0.511492 \\
\hline 15 & 1 & 0 & 0.220521 & -2.064140 & -1.489992 \\
\hline 16 & 1 & 0 & 2. 044008 & -3.764601 & 1. 631857 \\
\hline 17 & 1 & 0 & -1.561274 & -4.107416 & -0.751459 \\
\hline 18 & 15 & 0 & -1.489903 & -0.926291 & -0.250619 \\
\hline 19 & 15 & 0 & 1. 884635 & -0.580849 & 0.291587 \\
\hline 20 & 6 & 0 & 2. 987616 & -0.379160 & 1. 787328 \\
\hline 21 & 6 & 0 & 4. 246169 & 0.252332 & 1. 671020 \\
\hline 22 & 6 & 0 & 2. 537619 & -0.782617 & 3. 064689 \\
\hline 23 & 6 & 0 & 5. 045773 & 0.461624 & 2. 809470 \\
\hline 24 & 1 & 0 & 4. 615019 & 0.568554 & 0.699014 \\
\hline 25 & 6 & 0 & 3. 339077 & -0.572519 & 4. 201192 \\
\hline 26 & 1 & 0 & 1. 570528 & -1.261741 & 3. 191973 \\
\hline 27 & 6 & 0 & 4. 596480 & 0.049583 & 4. 078354 \\
\hline 28 & 1 & 0 & 6. 015327 & 0.941423 & 2. 703773 \\
\hline 29 & 1 & 0 & 2. 983495 & -0.895426 & 5. 176170 \\
\hline 30 & 1 & 0 & 5. 216039 & 0.208695 & 4. 956788 \\
\hline 31 & 6 & 0 & 3. 021742 & -0.820350 & -1.170832 \\
\hline 32 & 6 & 0 & 2. 665480 & -0.222934 & -2.398603 \\
\hline 33 & 6 & 0 & 4. 206090 & -1.587329 & -1.089227 \\
\hline 34 & 6 & 0 & 3. 473631 & -0.400571 & -3.537409 \\
\hline 35 & 1 & 0 & 1. 769482 & 0.390030 & -2.463404 \\
\hline 36 & 6 & 0 & 5. 016942 & -1.756559 & -2.225310 \\
\hline 37 & 1 & 0 & 4. 500554 & -2.048270 & -0.150078 \\
\hline 38 & 6 & 0 & 4. 650550 & -1.167577 & -3.453096 \\
\hline 39 & 1 & 0 & 3. 189050 & 0.062222 & -4.478703 \\
\hline 40 & 1 & 0 & 5. 926384 & -2.347338 & -2.154627 \\
\hline 41 & 1 & 0 & 5.278505 & -1.302680 & -4.329853 \\
\hline 42 & 6 & 0 & -2.474118 & -1.393632 & 1. 266958 \\
\hline 43 & 6 & 0 & -2.222938 & -0.714611 & 2. 477957 \\
\hline 44 & 6 & 0 & -3.426934 & -2.436246 & 1. 237299 \\
\hline 45 & 6 & 0 & -2.906891 & -1.080834 & 3. 652337 \\
\hline 46 & 1 & 0 & -1.508024 & 0.104948 & 2. 499814 \\
\hline 47 & 6 & 0 & -4.112300 & -2.798018 & 2. 410479 \\
\hline 48 & 1 & 0 & -3.645803 & -2.955364 & 0.307968 \\
\hline 49 & 6 & 0 & -3.851129 & -2.124251 & 3. 621081 \\
\hline 50 & 1 & 0 & -2.710889 & -0.550366 & 4. 580614 \\
\hline 51 & 1 & 0 & -4.845814 & -3.599363 & 2. 380780 \\
\hline 52 & 1 & 0 & -4.383369 & -2.405816 & 4. 525924 \\
\hline 53 & 6 & 0 & -2.684218 & -1.039460 & -1.680511 \\
\hline 54 & 6 & 0 & -2.282959 & -1.574557 & -2.924593 \\
\hline 55 & 6 & 0 & -3.991060 & -0.521154 & -1.539650 \\
\hline 56 & 6 & 0 & -3.180607 & -1.604118 & -4.007643 \\
\hline 57 & 1 & 0 & -1.283310 & -1.976999 & -3.063663 \\
\hline 58 & 6 & 0 & -4.886732 & -0.555449 & -2.623597 \\
\hline 59 & 1 & 0 & -4.317215 & -0.099326 & -0.593033 \\
\hline 60 & 6 & 0 & -4.485610 & -1.096156 & -3.860536 \\
\hline 61 & 1 & 0 & -2.863078 & -2.025401 & -4.957922 \\
\hline 62 & 1 & 0 & -5.892505 & -0.162510 & -2.500180 \\
\hline 63 & 1 & 0 & -5.179757 & -1.123777 & -4.696307 \\
\hline
\end{tabular}




$\begin{array}{rrrrrr}64 & 46 & 0 & 0.023870 & 0.993130 & -0.038333 \\ 65 & 6 & 0 & -1.347568 & 2.771446 & -0.402616 \\ 66 & 6 & 0 & -0.177724 & 3.198207 & 0.293451 \\ 67 & 6 & 0 & 1.111388 & 2.957990 & -0.246390 \\ 68 & 1 & 0 & -0.260626 & 3.554709 & 1.319597 \\ 69 & 1 & 0 & 1.221760 & 2.896626 & -1.330326 \\ 70 & 1 & 0 & -1.282913 & 2.716139 & -1.491977 \\ 71 & 6 & 0 & -2.720769 & 2.907578 & 0.129377 \\ 72 & 6 & 0 & -2.999437 & 3.010235 & 1.516481 \\ 73 & 6 & 0 & -3.803896 & 2.974705 & -0.781273 \\ 74 & 6 & 0 & -4.313136 & 3.191682 & 1.972469 \\ 75 & 1 & 0 & -2.192163 & 2.946375 & 2.242826 \\ 76 & 6 & 0 & -5.120562 & 3.158039 & -0.325156 \\ 77 & 1 & 0 & -3.609531 & 2.899244 & -1.849367 \\ 78 & 6 & 0 & -5.381403 & 3.268729 & 1.054211 \\ 79 & 1 & 0 & -4.509151 & 3.272539 & 3.038613 \\ 80 & 1 & 0 & -5.936300 & 3.223669 & -1.040519 \\ 81 & 1 & 0 & -6.398035 & 3.414518 & 1.409675 \\ 82 & 6 & 0 & 2.372920 & 3.372861 & 0.484579 \\ 83 & 1 & 0 & 3.163750 & 2.627529 & 0.333517 \\ 84 & 1 & 0 & 2.180950 & 3.419487 & 1.565339 \\ 85 & 6 & 0 & 2.899523 & 4.755146 & -0.002800 \\ 86 & 1 & 0 & 2.064222 & 5.461067 & -0.101429 \\ 87 & 1 & 0 & 3.578832 & 5.161404 & 0.760311 \\ 88 & 6 & 0 & 3.674624 & 4.702607 & -1.322672 \\ 89 & 1 & 0 & 4.563299 & 4.060134 & -1.216582 \\ 90 & 1 & 0 & 4.001674 & 5.716021 & -1.598685 \\ 91 & 8 & 0 & 2.792436 & 4.154075 & -2.365632 \\ 92 & 1 & 0 & 3.186886 & 4.276861 & -3.253426 \\ -----------------------------------------------------------\end{array}$




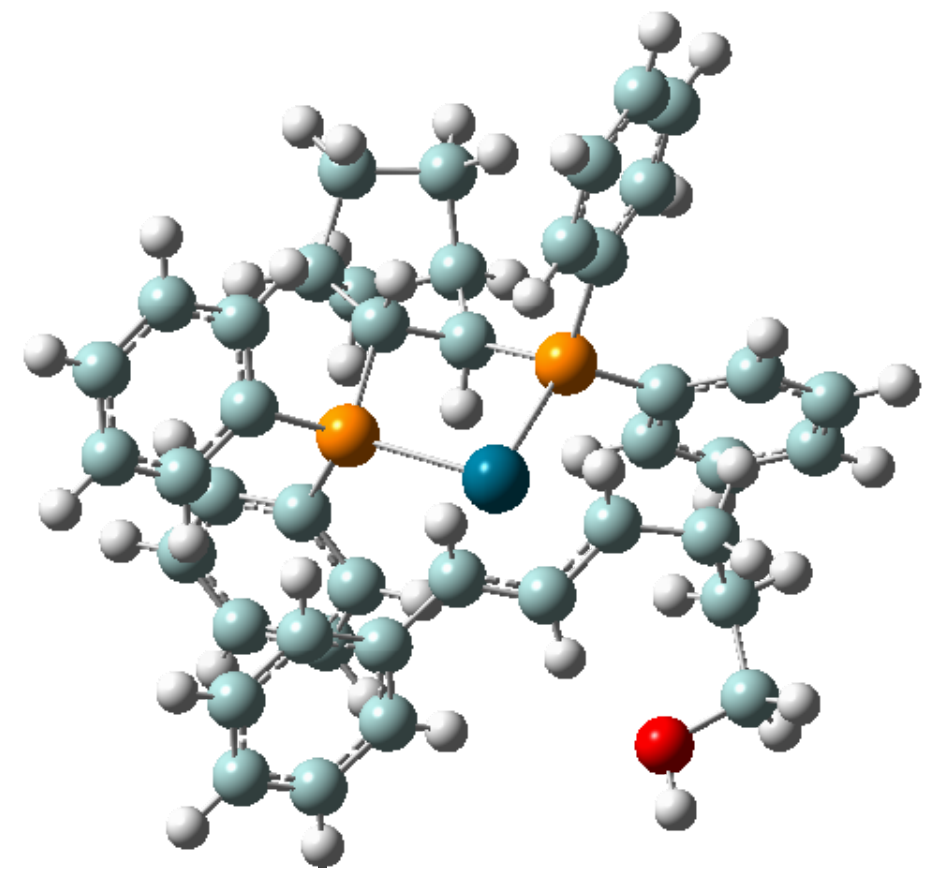

\section{$\pi$-Allyl intermediate in hydroalkoxylation, isomer II}

$\mathrm{E}(\mathrm{RB}+\mathrm{HF}-\mathrm{LYP})=-2551.06430792 \quad$ A. $\mathrm{U}$.

Standard orientation:

\begin{tabular}{rrrrrr} 
Center & Atomic & Atomic & \multicolumn{3}{c}{ Coordinates (Angstroms) } \\
Number & Number & Type & X & Y & $Z$ \\
-----1 & & 0 & 2.504416 & -3.910438 & 0.992506 \\
1 & 6 & 0 & 2.066722 & -2.745574 & 1.933819 \\
2 & 6 & 0 & 0.084000 & -3.685393 & 1.265295 \\
3 & 6 & 0 & 1.149224 & -4.619258 & 0.616155 \\
4 & 6 & 0 & 3.045659 & -3.560304 & 0.110696 \\
5 & 1 & 0 & 3.169582 & -4.593270 & 1.533072 \\
6 & 1 & 0 & 1.015594 & -4.723745 & -0.467180 \\
7 & 1 & 0 & 1.092787 & -5.623110 & 1.053176 \\
8 & 1 & 0 & 0.795425 & -3.357833 & 2.612052 \\
9 & 6 & 0 & 0.254043 & -2.651319 & 3.249878 \\
10 & 1 & 0 & 1.013765 & -4.260247 & 3.193656 \\
11 & 1 & 0 & 1.346618 & -1.593242 & 1.172083 \\
12 & 6 & 0 & 0.921008 & -0.947733 & 1.955364 \\
13 & 1 & & & &
\end{tabular}




\begin{tabular}{|c|c|c|c|c|c|}
\hline 14 & 6 & 0 & 0.169737 & -2.340241 & 0.464751 \\
\hline 15 & 1 & 0 & 0.451508 & -2.570706 & -0.571114 \\
\hline 16 & 1 & 0 & 2. 850916 & -2.397517 & 2. 611528 \\
\hline 17 & 1 & 0 & -0.914325 & -4.123803 & 1. 329890 \\
\hline 18 & 15 & 0 & -1.264084 & -1.109604 & 0.246885 \\
\hline 19 & 15 & 0 & 2. 059941 & -0.286954 & -0.011806 \\
\hline 20 & 6 & 0 & 3. 344036 & 0.650558 & 0.971336 \\
\hline 21 & 6 & 0 & 4. 449242 & 1. 242981 & 0.320549 \\
\hline 22 & 6 & 0 & 3. 180800 & 0.843264 & 2. 361265 \\
\hline 23 & 6 & 0 & 5.382600 & 2. 001272 & 1. 050445 \\
\hline 24 & 1 & 0 & 4. 597041 & 1. 105849 & -0.747383 \\
\hline 25 & 6 & 0 & 4. 114955 & 1. 602622 & 3. 089004 \\
\hline 26 & 1 & 0 & 2. 337100 & 0.408693 & 2. 890648 \\
\hline 27 & 6 & 0 & 5. 219823 & 2. 183630 & 2. 437268 \\
\hline 28 & 1 & 0 & 6. 234032 & 2. 442242 & 0.538474 \\
\hline 29 & 1 & 0 & 3. 981225 & 1. 735961 & 4. 159351 \\
\hline 30 & 1 & 0 & 5. 943644 & 2. 765573 & 3. 001502 \\
\hline 31 & 6 & 0 & 2. 992096 & -1.130574 & -1.396091 \\
\hline 32 & 6 & 0 & 2. 369318 & -1.232573 & -2.658611 \\
\hline 33 & 6 & 0 & 4. 282805 & -1.675889 & -1.213818 \\
\hline 34 & 6 & 0 & 3. 021476 & -1.880322 & -3.724921 \\
\hline 35 & 1 & 0 & 1. 383429 & -0.798593 & -2.812152 \\
\hline 36 & 6 & 0 & 4. 936300 & -2.316446 & -2.281411 \\
\hline 37 & 1 & 0 & 4. 780820 & -1.601093 & -0.250866 \\
\hline 38 & 6 & 0 & 4. 306337 & -2.423515 & -3.538248 \\
\hline 39 & 1 & 0 & 2. 533208 & -1.952960 & -4.693215 \\
\hline 40 & 1 & 0 & 5. 929598 & -2.731708 & -2.133079 \\
\hline 41 & 1 & 0 & 4. 814261 & -2.919378 & -4.361175 \\
\hline 42 & 6 & 0 & -1.990614 & -0.836671 & 1. 945663 \\
\hline 43 & 6 & 0 & -1.752098 & 0.393376 & 2. 594645 \\
\hline 44 & 6 & 0 & -2.732223 & -1.841024 & 2. 607281 \\
\hline 45 & 6 & 0 & -2.239816 & 0.615887 & 3. 896125 \\
\hline 46 & 1 & 0 & -1.200200 & 1. 177589 & 2. 081179 \\
\hline 47 & 6 & 0 & -3.221418 & -1.615780 & 3. 906023 \\
\hline 48 & 1 & 0 & -2.941000 & -2.787064 & 2. 114894 \\
\hline 49 & 6 & 0 & -2.973198 & -0.388776 & 4. 554772 \\
\hline 50 & 1 & 0 & -2.055593 & 1. 567972 & 4. 387102 \\
\hline 51 & 1 & 0 & -3.793318 & -2.391341 & 4. 408550 \\
\hline 52 & 1 & 0 & -3.353985 & -0.217390 & 5. 558253 \\
\hline 53 & 6 & 0 & -2.570518 & -2.016804 & -0.730541 \\
\hline 54 & 6 & 0 & -2.209980 & -2.994136 & -1.685140 \\
\hline 55 & 6 & 0 & -3.932238 & -1.680224 & -0.566825 \\
\hline 56 & 6 & 0 & -3.198969 & -3.638401 & -2.450206 \\
\hline 57 & 1 & 0 & -1.170020 & -3.270251 & -1.838089 \\
\hline 58 & 6 & 0 & -4.919080 & -2.328799 & -1.331543 \\
\hline 59 & 1 & 0 & -4.228785 & -0.923079 & 0. 153325 \\
\hline 60 & 6 & 0 & -4.557255 & -3.310080 & -2.273960 \\
\hline 61 & 1 & 0 & -2.910129 & -4.395101 & -3.174998 \\
\hline 62 & 1 & 0 & -5.964316 & -2.067177 & -1.189413 \\
\hline 63 & 1 & 0 & -5.321090 & -3.812593 & -2.861462 \\
\hline 64 & 46 & 0 & -0.041230 & 0.795796 & -0.702777 \\
\hline 65 & 6 & 0 & -1.705191 & 1.982742 & -1.722158 \\
\hline
\end{tabular}




$\begin{array}{rrrrrr}66 & 6 & 0 & -0.610711 & 2.843908 & -1.426881 \\ 67 & 6 & 0 & 0.690050 & 2.546201 & -1.911491 \\ 68 & 1 & 0 & -0.713536 & 3.650567 & -0.704667 \\ 69 & 1 & 0 & 0.767272 & 1.978582 & -2.845108 \\ 70 & 1 & 0 & -1.658364 & 1.395065 & -2.643269 \\ 71 & 6 & 0 & -3.068541 & 2.179444 & -1.184919 \\ 72 & 6 & 0 & -3.328102 & 2.959719 & -0.028331 \\ 73 & 6 & 0 & -4.165614 & 1.601249 & -1.869162 \\ 74 & 6 & 0 & -4.642499 & 3.162756 & 0.415731 \\ 75 & 1 & 0 & -2.505859 & 3.412180 & 0.520652 \\ 76 & 6 & 0 & -5.483191 & 1.807673 & -1.425787 \\ 77 & 1 & 0 & -3.985084 & 1.000444 & -2.758462 \\ 78 & 6 & 0 & -5.727876 & 2.590035 & -0.281079 \\ 79 & 1 & 0 & -4.826507 & 3.766976 & 1.300527 \\ 80 & 1 & 0 & -6.312901 & 1.368360 & -1.973588 \\ 81 & 1 & 0 & -6.745865 & 2.754846 & 0.062293 \\ 82 & 6 & 0 & 1.920625 & 3.386594 & -1.602793 \\ 83 & 1 & 0 & 1.989734 & 4.173241 & -2.374702 \\ 84 & 1 & 0 & 2.801437 & 2.750802 & -1.761184 \\ 85 & 6 & 0 & 2.034384 & 4.045561 & -0.200666 \\ 86 & 1 & 0 & 1.587574 & 3.399229 & 0.566123 \\ 87 & 1 & 0 & 3.102384 & 4.118294 & 0.046143 \\ 88 & 6 & 0 & 1.463781 & 5.460938 & -0.048605 \\ 89 & 1 & 0 & 1.774898 & 6.093025 & -0.895118 \\ 90 & 1 & 0 & 1.846019 & 5.905658 & 0.882171 \\ 91 & 8 & 0 & -0.004823 & 5.396044 & 0.016667 \\ 92 & 1 & 0 & -0.394166 & 6.291220 & 0.094463 \\ ------------------------------------------1\end{array}$




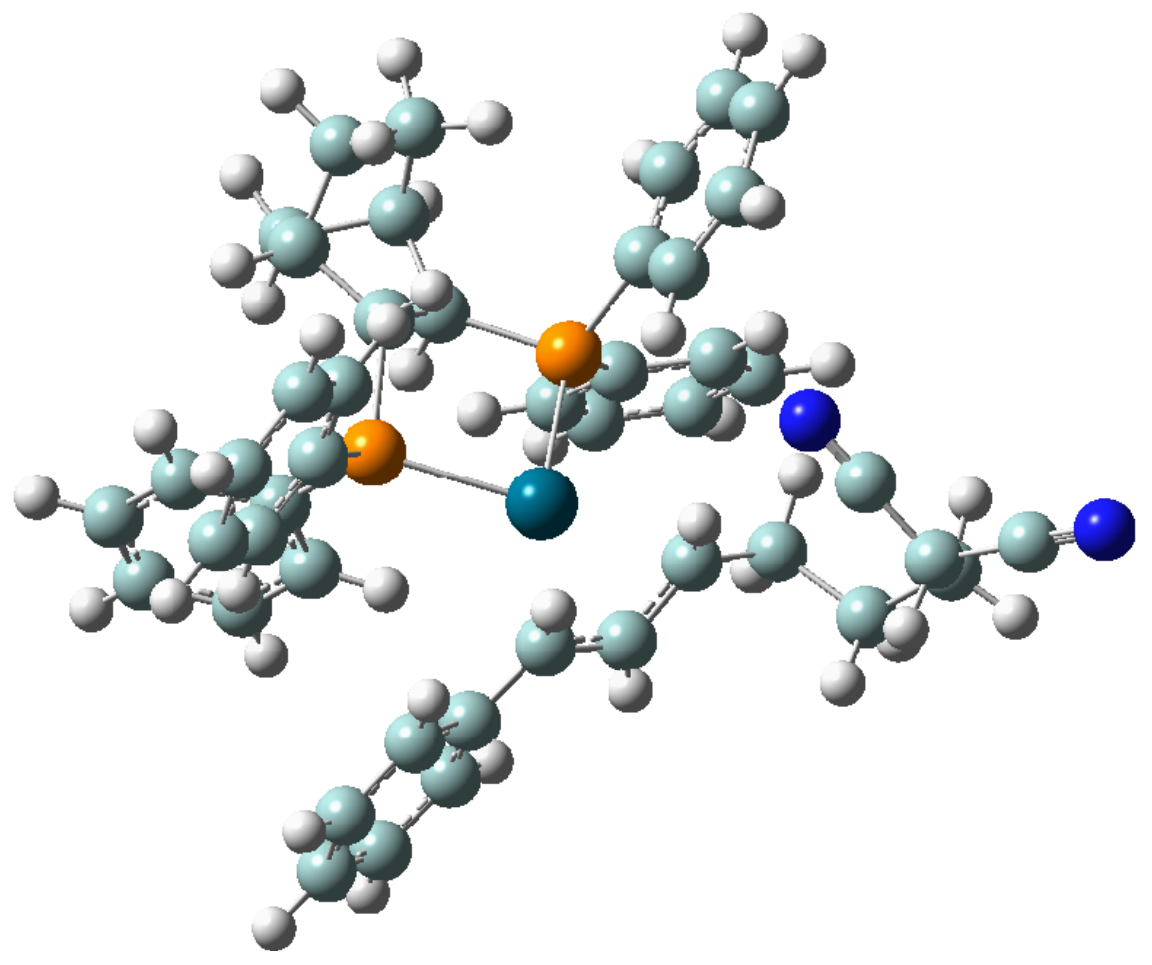

\section{$\pi$-Allyl intermediate in hydrocarbonation, isomer I}

$\mathrm{E}(\mathrm{RB}+\mathrm{HF}-\mathrm{LYP})=-2699.57762784 \quad$ A. $\mathrm{U}$.

Standard orientation:

\begin{tabular}{cccccc} 
Center & Atomic & Atomic & \multicolumn{3}{c}{ Coordinates (Angstroms) } \\
Number & Number & Type & X & Y & $Z$ \\
----1 & 6 & 0 & -0.515349 & -4.580732 & -1.666712 \\
1 & 6 & 0 & -0.959632 & -4.135069 & -0.237646 \\
2 & 6 & 0 & -2.444490 & -3.079513 & -1.632136 \\
3 & 6 & 0 & -1.600579 & -3.944881 & -2.614662 \\
4 & 1 & 0 & 0.494980 & -4.250023 & -1.916356 \\
5 & 1 & 0 & -0.525589 & -5.674479 & -1.733493 \\
6 & 1 & 0 & -1.158008 & -3.354101 & -3.425355 \\
7 & 1 & 0 & -2.227344 & -4.717825 & -3.074387 \\
8 & 6 & 0 & -2.512890 & -4.046403 & -0.411846 \\
9 & 1 & 0 & -3.031422 & -3.639773 & 0.462618 \\
10 & 1 & 0 & -2.968022 & -5.007762 & -0.673834 \\
11 & 6 & 0 & -0.703755 & -2.620968 & 0.025533 \\
12 & & & & &
\end{tabular}




\begin{tabular}{|c|c|c|c|c|c|}
\hline 13 & 1 & 0 & -1.239153 & -2.393686 & 0.958843 \\
\hline 14 & 6 & 0 & -1.479064 & -1.942783 & -1.150059 \\
\hline 15 & 1 & 0 & -0.782719 & -1.706687 & -1.965538 \\
\hline 16 & 1 & 0 & -0.604997 & -4.784081 & 0.567682 \\
\hline 17 & 1 & 0 & -3.396574 & -2.736625 & -2.043499 \\
\hline 18 & 15 & 0 & -2.032971 & -0.222349 & -0.560191 \\
\hline 19 & 15 & 0 & 0.932095 & -1.702173 & 0.324065 \\
\hline 20 & 6 & 0 & 1. 571217 & -2.318608 & 1. 973125 \\
\hline 21 & 6 & 0 & 2. 961410 & -2.357520 & 2. 222990 \\
\hline 22 & 6 & 0 & 0.677627 & -2.675915 & 3. 007944 \\
\hline 23 & 6 & 0 & 3. 448274 & -2.760917 & 3. 479767 \\
\hline 24 & 1 & 0 & 3. 667604 & -2.092460 & 1. 440779 \\
\hline 25 & 6 & 0 & 1. 167260 & -3.079917 & 4. 263468 \\
\hline 26 & 1 & 0 & -0.397948 & -2.651828 & 2. 854457 \\
\hline 27 & 6 & 0 & 2. 553807 & -3.124504 & 4. 504463 \\
\hline 28 & 1 & 0 & 4. 520525 & -2.795813 & 3. 654315 \\
\hline 29 & 1 & 0 & 0.468410 & -3.361628 & 5. 046775 \\
\hline 30 & 1 & 0 & 2. 930555 & -3.440698 & 5. 473458 \\
\hline 31 & 6 & 0 & 2. 211272 & -2.212470 & -0.933542 \\
\hline 32 & 6 & 0 & 2. 604377 & -1.283723 & -1.918653 \\
\hline 33 & 6 & 0 & 2. 794153 & -3.500818 & -0.915512 \\
\hline 34 & 6 & 0 & 3. 566532 & -1.636282 & -2.884462 \\
\hline 35 & 1 & 0 & 2. 192166 & -0.279137 & -1.928891 \\
\hline 36 & 6 & 0 & 3. 751358 & -3.852273 & -1.882405 \\
\hline 37 & 1 & 0 & 2. 513384 & -4.223117 & -0.153135 \\
\hline 38 & 6 & 0 & 4. 136978 & -2.922100 & -2.871200 \\
\hline 39 & 1 & 0 & 3. 871430 & -0.901362 & -3.623338 \\
\hline 40 & 1 & 0 & 4. 195408 & -4.844075 & -1.864638 \\
\hline 41 & 1 & 0 & 4. 880823 & -3.196814 & -3.614485 \\
\hline 42 & 6 & 0 & -3.406404 & -0.487269 & 0.675510 \\
\hline 43 & 6 & 0 & -3.155593 & -0.233973 & 2. 040524 \\
\hline 44 & 6 & 0 & -4.673337 & -0.970888 & 0.277918 \\
\hline 45 & 6 & 0 & -4.155152 & -0.473054 & 3. 002270 \\
\hline 46 & 1 & 0 & -2.191474 & 0.163048 & 2. 349542 \\
\hline 47 & 6 & 0 & -5.671317 & -1.206277 & 1. 239642 \\
\hline 48 & 1 & 0 & -4.890304 & -1.151992 & -0.771357 \\
\hline 49 & 6 & 0 & -5.413428 & -0.961837 & 2. 604117 \\
\hline 50 & 1 & 0 & -3.955750 & -0.270693 & 4. 051383 \\
\hline 51 & 1 & 0 & -6.643989 & -1.576645 & 0.927067 \\
\hline 52 & 1 & 0 & -6.187772 & -1.143540 & 3. 344780 \\
\hline 53 & 6 & 0 & -2.813369 & 0.614587 & -2.034534 \\
\hline 54 & 6 & 0 & -2.316217 & 0.379780 & -3.336220 \\
\hline 55 & 6 & 0 & -3.861718 & 1. 542842 & -1.850953 \\
\hline 56 & 6 & 0 & -2.874051 & 1. 049328 & -4.440124 \\
\hline 57 & 1 & 0 & -1.502405 & -0.320153 & -3.506775 \\
\hline 58 & 6 & 0 & -4.419098 & 2. 208640 & -2.958145 \\
\hline 59 & 1 & 0 & -4.249419 & 1. 745381 & -0.856697 \\
\hline 60 & 6 & 0 & -3.929353 & 1. 963859 & -4.255239 \\
\hline 61 & 1 & 0 & -2.488281 & 0.855473 & -5.437463 \\
\hline 62 & 1 & 0 & -5.232842 & 2. 912867 & -2.806023 \\
\hline 63 & 1 & 0 & -4.362908 & 2. 476739 & -5.109628 \\
\hline 64 & 46 & 0 & 0.108355 & 0.612265 & 0.279985 \\
\hline
\end{tabular}




\begin{tabular}{rrrrrr}
65 & 6 & 0 & -0.261185 & 2.860637 & 0.350567 \\
66 & 6 & 0 & 0.860862 & 2.525541 & 1.165961 \\
67 & 6 & 0 & 1.969428 & 1.830551 & 0.615122 \\
68 & 1 & 0 & 0.796900 & 2.642643 & 2.247231 \\
69 & 1 & 0 & 2.153562 & 1.948980 & -0.453875 \\
70 & 1 & 0 & -0.074575 & 3.015218 & -0.714993 \\
71 & 6 & 0 & -1.493700 & 3.498853 & 0.859756 \\
72 & 6 & 0 & -1.880021 & 3.444629 & 2.223745 \\
73 & 6 & 0 & -2.309194 & 4.225366 & -0.042724 \\
74 & 6 & 0 & -3.031199 & 4.109856 & 2.669556 \\
75 & 1 & 0 & -1.284851 & 2.882950 & 2.940613 \\
76 & 6 & 0 & -3.462504 & 4.892593 & 0.403735 \\
77 & 1 & 0 & -2.029192 & 4.278655 & -1.092945 \\
78 & 6 & 0 & -3.828132 & 4.839549 & 1.762453 \\
79 & 1 & 0 & -3.310524 & 4.063775 & 3.719061 \\
80 & 1 & 0 & -4.067104 & 5.457246 & -0.301168 \\
81 & 1 & 0 & -4.716807 & 5.358808 & 2.111267 \\
82 & 6 & 0 & 3.164495 & 1.418804 & 1.456115 \\
83 & 1 & 0 & 3.690883 & 0.588535 & 0.968570 \\
84 & 1 & 0 & 2.804941 & 1.036771 & 2.420323 \\
85 & 6 & 0 & 4.185334 & 2.572336 & 1.749627 \\
86 & 1 & 0 & 3.658254 & 3.537482 & 1.783419 \\
87 & 1 & 0 & 4.573608 & 2.408092 & 2.763799 \\
88 & 6 & 0 & 5.434429 & 2.687125 & 0.840069 \\
89 & 1 & 0 & 5.895861 & 1.703161 & 0.690726 \\
90 & 1 & 0 & 6.174922 & 3.309367 & 1.357911 \\
91 & 6 & 0 & 5.219539 & 3.365160 & -0.562327 \\
92 & 1 & 0 & 4.624231 & 4.279294 & -0.417650 \\
93 & 6 & 0 & 4.492148 & 2.500608 & -1.518728 \\
94 & 6 & 0 & 6.518300 & 3.766883 & -1.150216 \\
95 & 7 & 7.563397 & 4.094504 & -1.586250 \\
96 & 7 & 3.898305 & 1.794232 & -2.251197 \\
------------------------------------------------ \\
& 6 & & & & \\
\hline 5 & & 0 & 0 & 0 & 0
\end{tabular}




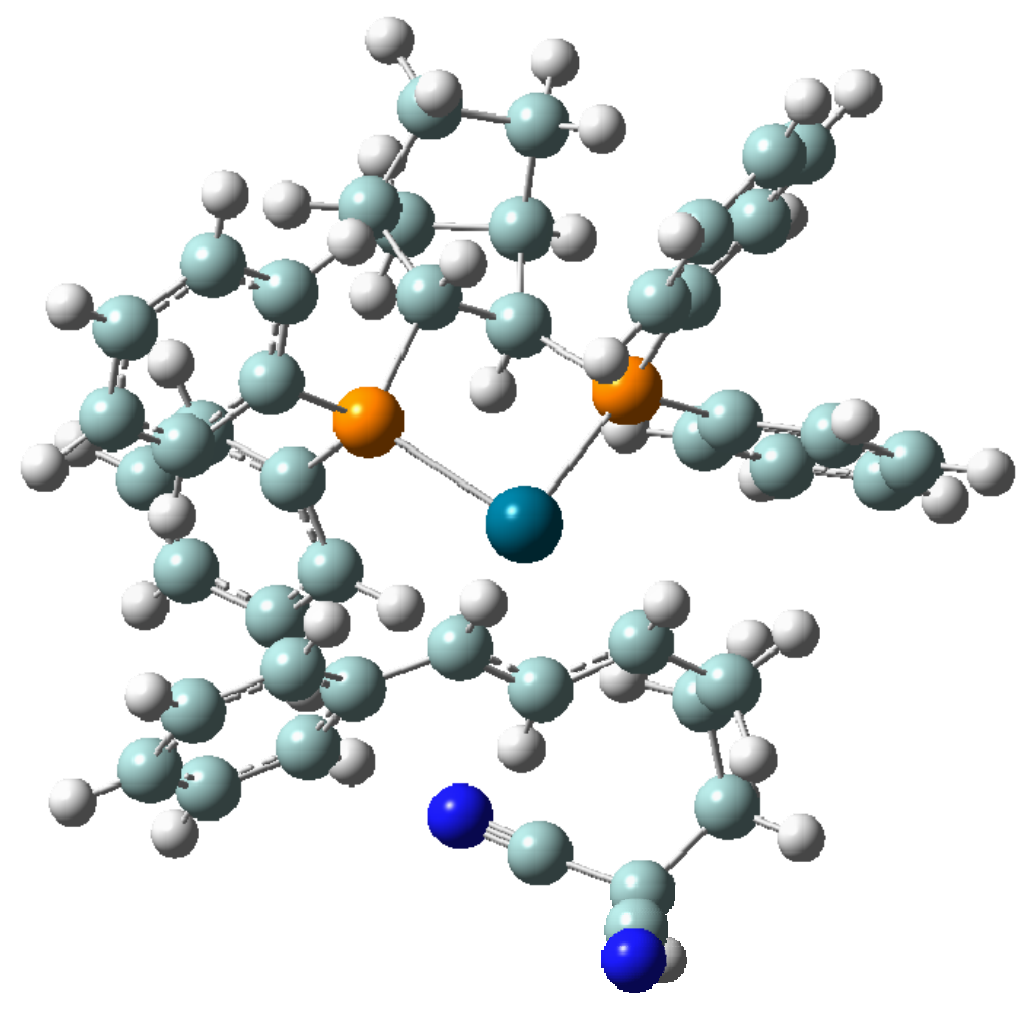

$\pi$-Allyl intermediate in hydrocarbonation, isomer II

$\mathrm{E}(\mathrm{RB}+\mathrm{HF}-\mathrm{LYP})=-2699.57613045 \quad$ A. U.

Standard orientation:

\begin{tabular}{|c|c|c|c|c|c|}
\hline \multirow{2}{*}{$\begin{array}{l}\text { Center } \\
\text { Number }\end{array}$} & \multirow{2}{*}{$\begin{array}{l}\text { Atomic } \\
\text { Number }\end{array}$} & \multirow{2}{*}{$\begin{array}{l}\text { Atomic } \\
\text { Type }\end{array}$} & \multicolumn{3}{|c|}{ Coordinates (Angstroms) } \\
\hline & & & X & $\mathrm{Y}$ & Z \\
\hline 1 & 6 & 0 & 3. 074103 & -3.671104 & 1. 479117 \\
\hline 2 & 6 & 0 & 2. 531601 & -2.407800 & 2. 217253 \\
\hline 3 & 6 & 0 & 0.652623 & -3.661228 & 1. 822653 \\
\hline 4 & 6 & 0 & 1. 799756 & -4.580153 & 1. 304825 \\
\hline 5 & 1 & 0 & 3.543495 & -3.431414 & 0.522013 \\
\hline 6 & 1 & 0 & 3. 830572 & -4.166340 & 2. 098092 \\
\hline 7 & 1 & 0 & 1. 649995 & -4.900052 & 0.266758 \\
\hline 8 & 1 & 0 & 1. 870642 & -5.484119 & 1. 920961 \\
\hline 9 & 6 & 0 & 1. 356644 & -3.016326 & 3. 053496 \\
\hline
\end{tabular}




\begin{tabular}{|c|c|c|c|c|c|}
\hline 10 & 1 & 0 & 0.754952 & -2.266515 & 3. 577802 \\
\hline 11 & 1 & 0 & 1. 691867 & -3.767032 & 3. 777507 \\
\hline 12 & 6 & 0 & 1. 664580 & -1.496827 & 1. 296686 \\
\hline 13 & 1 & 0 & 1. 188389 & -0.766864 & 1. 969587 \\
\hline 14 & 6 & 0 & 0.564838 & -2.485134 & 0.790520 \\
\hline 15 & 1 & 0 & 0.849970 & -2.870981 & -0.197065 \\
\hline 16 & 1 & 0 & 3. 290653 & -1.863431 & 2. 785031 \\
\hline 17 & 1 & 0 & -0.288756 & -4.182184 & 2. 010202 \\
\hline 18 & 15 & 0 & -1.004241 & -1.487322 & 0.408126 \\
\hline 19 & 15 & 0 & 2. 185215 & -0.360794 & -0.138432 \\
\hline 20 & 6 & 0 & 3. 490794 & 0.782626 & 0.550560 \\
\hline 21 & 6 & 0 & 4. 421120 & 1. 395280 & -0.318896 \\
\hline 22 & 6 & 0 & 3. 518008 & 1. 102354 & 1. 925808 \\
\hline 23 & 6 & 0 & 5. 370944 & 2. 303671 & 0.182637 \\
\hline 24 & 1 & 0 & 4. 419081 & 1. 160062 & -1.380316 \\
\hline 25 & 6 & 0 & 4. 468567 & 2. 011788 & 2. 425291 \\
\hline 26 & 1 & 0 & 2. 810034 & 0.654039 & 2. 617117 \\
\hline 27 & 6 & 0 & 5. 398482 & 2. 615083 & 1. 556526 \\
\hline 28 & 1 & 0 & 6. 087732 & 2. 760694 & -0.494567 \\
\hline 29 & 1 & 0 & 4. 485113 & 2. 241985 & 3. 487347 \\
\hline 30 & 1 & 0 & 6. 136769 & 3. 311896 & 1. 944281 \\
\hline 31 & 6 & 0 & 3. 044494 & -1.359827 & -1.466137 \\
\hline 32 & 6 & 0 & 2. 313144 & -1.711014 & -2.621500 \\
\hline 33 & 6 & 0 & 4. 390339 & -1.772572 & -1.344819 \\
\hline 34 & 6 & 0 & 2. 913668 & -2.475487 & -3.639431 \\
\hline 35 & 1 & 0 & 1. 283142 & -1.378001 & -2.733194 \\
\hline 36 & 6 & 0 & 4. 990921 & -2.530890 & -2.365539 \\
\hline 37 & 1 & 0 & 4. 973191 & -1.501098 & -0.468706 \\
\hline 38 & 6 & 0 & 4. 253553 & -2.887304 & -3.513047 \\
\hline 39 & 1 & 0 & 2. 342936 & -2.738542 & -4.526192 \\
\hline 40 & 1 & 0 & 6. 027198 & -2.842589 & -2.265598 \\
\hline 41 & 1 & 0 & 4. 721311 & -3.472368 & -4.300392 \\
\hline 42 & 6 & 0 & -1.740631 & -0.939972 & 2. 034575 \\
\hline 43 & 6 & 0 & -1.691253 & 0.430193 & 2. 367881 \\
\hline 44 & 6 & 0 & -2.331255 & -1.854028 & 2. 936048 \\
\hline 45 & 6 & 0 & -2.222541 & 0.891243 & 3. 586429 \\
\hline 46 & 1 & 0 & -1.256269 & 1. 144898 & 1. 674554 \\
\hline 47 & 6 & 0 & -2.855755 & -1.395050 & 4. 157584 \\
\hline 48 & 1 & 0 & -2.400127 & -2.910036 & 2. 687555 \\
\hline 49 & 6 & 0 & -2.801266 & -0.023997 & 4. 485527 \\
\hline 50 & 1 & 0 & -2.191725 & 1. 953616 & 3. 809997 \\
\hline 51 & 1 & 0 & -3.309741 & -2.101738 & 4. 847409 \\
\hline 52 & 1 & 0 & -3.215956 & 0.324823 & 5. 427861 \\
\hline 53 & 6 & 0 & -2.214284 & -2.703338 & -0.326301 \\
\hline 54 & 6 & 0 & -1.760738 & -3.821767 & -1.061243 \\
\hline 55 & 6 & 0 & -3.602064 & -2.473821 & -0.201553 \\
\hline 56 & 6 & 0 & -2.683045 & -4.707677 & -1.646795 \\
\hline 57 & 1 & 0 & -0.699023 & -4.021555 & -1.178956 \\
\hline 58 & 6 & 0 & -4.521211 & -3.363873 & -0.786153 \\
\hline 59 & 1 & 0 & -3.970702 & -1.613014 & 0.349497 \\
\hline 60 & 6 & 0 & -4.066577 & -4.483176 & -1.508754 \\
\hline 61 & 1 & 0 & -2.322572 & -5.569516 & -2.202384 \\
\hline
\end{tabular}




\begin{tabular}{|c|c|c|c|c|c|}
\hline 62 & 1 & 0 & -5.586693 & -3.179911 & -0.677370 \\
\hline 63 & 1 & 0 & -4.778705 & -5.170889 & -1.957158 \\
\hline 64 & 46 & 0 & -0.048512 & 0.411454 & -0.827685 \\
\hline 65 & 6 & 0 & -1.891538 & 1. 221408 & -1.967272 \\
\hline 66 & 6 & 0 & -1.015664 & 2. 273862 & -1.596136 \\
\hline 67 & 6 & 0 & 0.349843 & 2. 272659 & -2.006387 \\
\hline 68 & 1 & 0 & -1.354936 & 3. 001229 & -0.864113 \\
\hline 69 & 1 & 0 & 0.581344 & 1. 786678 & -2.958291 \\
\hline 70 & 1 & 0 & -1.638193 & 0.656751 & -2.868621 \\
\hline 71 & 6 & 0 & -3.304009 & 1. 113880 & -1.553996 \\
\hline 72 & 6 & 0 & -3.827192 & 1. 767829 & -0.408506 \\
\hline 73 & 6 & 0 & -4.187214 & 0.359426 & -2.365348 \\
\hline 74 & 6 & 0 & -5.192769 & 1. 682937 & -0.102270 \\
\hline 75 & 1 & 0 & -3.177309 & 2. 337746 & 0.250605 \\
\hline 76 & 6 & 0 & -5.556394 & 0.280013 & -2.061692 \\
\hline 77 & 1 & 0 & -3.801730 & -0.151459 & -3.245647 \\
\hline 78 & 6 & 0 & -6.065628 & 0.943740 & -0.929173 \\
\hline 79 & 1 & 0 & -5.579643 & 2. 191964 & 0.776500 \\
\hline 80 & 1 & 0 & -6.220979 & -0.289426 & -2.705899 \\
\hline 81 & 1 & 0 & -7.125199 & 0.889623 & -0.693222 \\
\hline 82 & 6 & 0 & 1. 315199 & 3. 386912 & -1.614179 \\
\hline 83 & 1 & 0 & 1. 022253 & 4. 286201 & -2.179349 \\
\hline 84 & 1 & 0 & 2. 312812 & 3. 120715 & -1.982282 \\
\hline 85 & 6 & 0 & 1. 415659 & 3. 720353 & -0.090509 \\
\hline 86 & 1 & 0 & 0.714647 & 3. 096691 & 0.479248 \\
\hline 87 & 1 & 0 & 2. 410905 & 3. 424214 & 0.256343 \\
\hline 88 & 6 & 0 & 1. 227701 & 5. 202069 & 0.313996 \\
\hline 89 & 1 & 0 & 1. 732705 & 5.870356 & -0.394489 \\
\hline 90 & 1 & 0 & 1. 688032 & 5. 365125 & 1. 296672 \\
\hline 91 & 6 & 0 & -0.261057 & 5. 695942 & 0.448753 \\
\hline 92 & 1 & 0 & -0.245408 & 6. 696747 & 0.904148 \\
\hline 93 & 6 & 0 & -1.023512 & 4. 795665 & 1. 346348 \\
\hline 94 & 6 & 0 & -0.948041 & 5.819105 & -0.857757 \\
\hline 95 & 7 & 0 & -1.473564 & 5. 930523 & -1.907332 \\
\hline 96 & 7 & 0 & -1.571696 & 4. 024309 & 2. 048770 \\
\hline
\end{tabular}




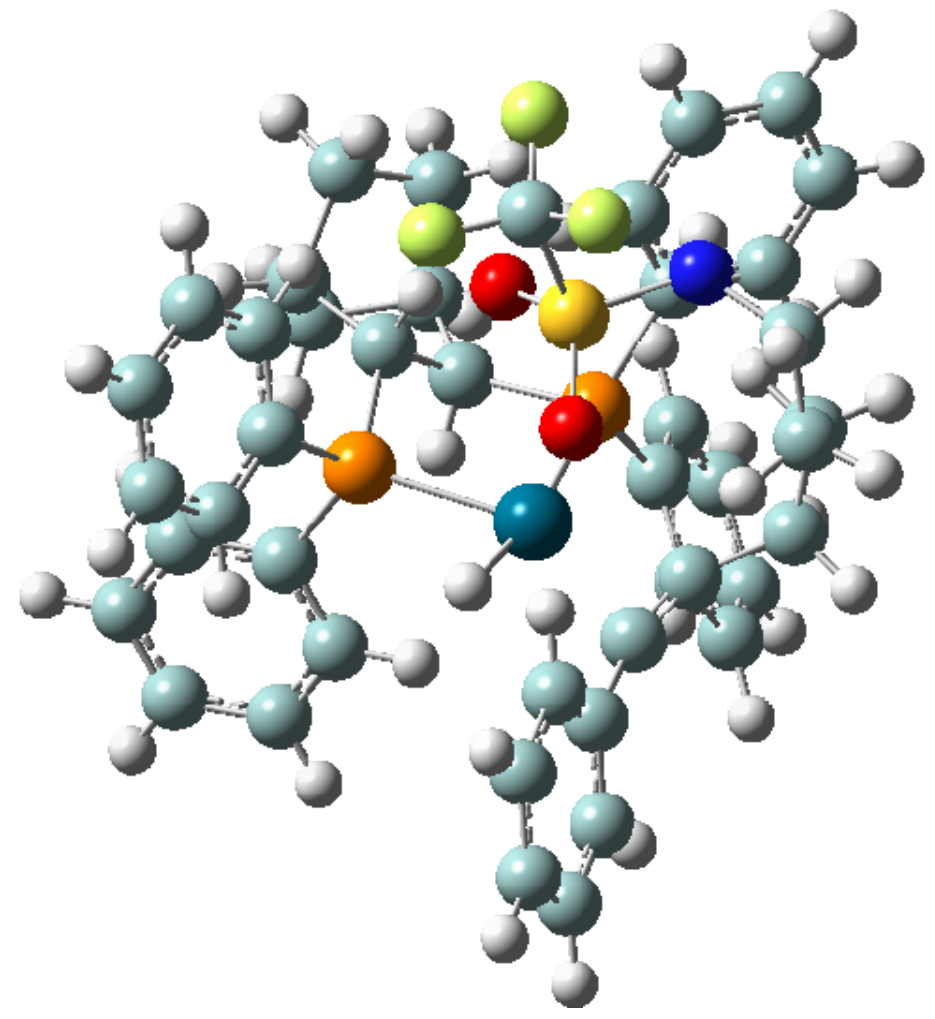

\section{Acetylenic intermediate of hydroamination, isomer I}

$\mathrm{E}(\mathrm{RB}+\mathrm{HF}-\mathrm{LYP})=-3416.54760696 \quad$ A. U.

Standard orientation:

\begin{tabular}{|c|c|c|c|c|c|}
\hline \multirow{2}{*}{$\begin{array}{l}\text { Center } \\
\text { Number }\end{array}$} & \multirow{2}{*}{$\begin{array}{l}\text { Atomic } \\
\text { Number }\end{array}$} & \multirow{2}{*}{$\begin{array}{c}\text { Atomic } \\
\text { Type }\end{array}$} & \multicolumn{3}{|c|}{ Coordinates (Angstroms) } \\
\hline & & & X & Y & Z \\
\hline 1 & 6 & 0 & -2.921019 & -1.989414 & -3.341883 \\
\hline 2 & 6 & 0 & -3.610286 & -1.043583 & -2.312593 \\
\hline 3 & 6 & 0 & -2.251092 & 0.364127 & -3.508682 \\
\hline 4 & 6 & 0 & -2.056047 & -1.009791 & -4.223268 \\
\hline 5 & 1 & 0 & -2.317685 & -2.764585 & -2.861261 \\
\hline 6 & 1 & 0 & -3.678837 & -2.497153 & -3.949087 \\
\hline 7 & 1 & 0 & -1.003002 & -1.310579 & -4.281153 \\
\hline 8 & 1 & 0 & -2.440260 & -0.959923 & -5.248712 \\
\hline 9 & 6 & 0 & -3.762195 & 0.271085 & -3.146368 \\
\hline 10 & 1 & 0 & -4.142592 & 1. 117022 & -2.565093 \\
\hline 11 & 1 & 0 & -4.391679 & 0.141769 & -4.033647 \\
\hline 12 & 6 & 0 & -2.615346 & -0.496255 & -1.247972 \\
\hline
\end{tabular}




\begin{tabular}{|c|c|c|c|c|c|}
\hline 13 & 1 & 0 & -3.177124 & 0.277838 & -0.701905 \\
\hline 14 & 6 & 0 & -1.529786 & 0.208534 & -2.126207 \\
\hline 15 & 1 & 0 & -0.654012 & -0.435770 & -2.258872 \\
\hline 16 & 1 & 0 & -4.535412 & -1.436064 & -1.883689 \\
\hline 17 & 1 & 0 & -1.939292 & 1. 225439 & -4.103986 \\
\hline 18 & 15 & 0 & -0.820143 & 1. 640864 & -1.101756 \\
\hline 19 & 15 & 0 & -1.819052 & -1.374981 & 0.246985 \\
\hline 20 & 6 & 0 & 0.860623 & 0.748135 & 2. 990692 \\
\hline 21 & 6 & 0 & 0.415063 & -0.419730 & 2. 850326 \\
\hline 22 & 6 & 0 & -3.283812 & -1.669466 & 1. 372014 \\
\hline 23 & 6 & 0 & -4.384276 & -2.460912 & 0.968982 \\
\hline 24 & 6 & 0 & -3.317000 & -1.029023 & 2. 629070 \\
\hline 25 & 6 & 0 & -5.494900 & -2.614893 & 1. 817758 \\
\hline 26 & 1 & 0 & -4.380618 & -2.960903 & 0.004086 \\
\hline 27 & 6 & 0 & -4.429716 & -1.183980 & 3. 477616 \\
\hline 28 & 1 & 0 & -2.484915 & -0.403628 & 2. 941982 \\
\hline 29 & 6 & 0 & -5.519475 & -1.978220 & 3. 075132 \\
\hline 30 & 1 & 0 & -6.335605 & -3.225902 & 1. 499937 \\
\hline 31 & 1 & 0 & -4.444847 & -0.686488 & 4. 443822 \\
\hline 32 & 1 & 0 & -6.378693 & -2.098831 & 3. 729533 \\
\hline 33 & 6 & 0 & -1.174342 & -3.070167 & -0.246791 \\
\hline 34 & 6 & 0 & -0.129313 & -3.154512 & -1.197760 \\
\hline 35 & 6 & 0 & -1.645958 & -4.254843 & 0.357355 \\
\hline 36 & 6 & 0 & 0.405102 & -4.404724 & -1.561513 \\
\hline 37 & 1 & 0 & 0.290173 & -2.259028 & -1.645064 \\
\hline 38 & 6 & 0 & -1.098868 & -5.504243 & 0.002252 \\
\hline 39 & 1 & 0 & -2.431991 & -4.218453 & 1. 104826 \\
\hline 40 & 6 & 0 & -0.079079 & -5.586466 & -0.963171 \\
\hline 41 & 1 & 0 & 1. 184718 & -4.456961 & -2.318394 \\
\hline 42 & 1 & 0 & -1.475113 & -6.407228 & 0.475939 \\
\hline 43 & 1 & 0 & 0. 331944 & -6.551998 & -1.245664 \\
\hline 44 & 6 & 0 & -2.157136 & 2. 927778 & -0.915074 \\
\hline 45 & 6 & 0 & -2.814031 & 3. 079526 & 0.323467 \\
\hline 46 & 6 & 0 & -2.533888 & 3. 734060 & -2.012273 \\
\hline 47 & 6 & 0 & -3.848021 & 4. 024256 & 0.463528 \\
\hline 48 & 1 & 0 & -2.509291 & 2. 478000 & 1. 176854 \\
\hline 49 & 6 & 0 & -3.566404 & 4. 677341 & -1.869208 \\
\hline 50 & 1 & 0 & -2.017944 & 3. 644632 & -2.964691 \\
\hline 51 & 6 & 0 & -4.227597 & 4. 821878 & -0.632348 \\
\hline 52 & 1 & 0 & -4.347310 & 4. 140576 & 1. 421765 \\
\hline 53 & 1 & 0 & -3.850005 & 5. 298474 & -2.714609 \\
\hline 54 & 1 & 0 & -5.023164 & 5. 554361 & -0.523831 \\
\hline 55 & 6 & 0 & 0.521386 & 2. 456742 & -2.101833 \\
\hline 56 & 6 & 0 & 1. 147570 & 1. 789262 & -3.173371 \\
\hline 57 & 6 & 0 & 0.943723 & 3. 755375 & -1.742546 \\
\hline 58 & 6 & 0 & 2. 178895 & 2. 423819 & -3.890319 \\
\hline 59 & 1 & 0 & 0.855808 & 0.782278 & -3.452781 \\
\hline 60 & 6 & 0 & 1. 974477 & 4. 385418 & -2.460116 \\
\hline 61 & 1 & 0 & 0.473904 & 4. 277570 & -0.912112 \\
\hline 62 & 6 & 0 & 2. 594037 & 3. 721263 & -3.537771 \\
\hline 63 & 1 & 0 & 2. 656923 & 1. 903762 & -4.716215 \\
\hline 64 & 1 & 0 & 2. 292230 & 5. 385969 & -2.179466 \\
\hline
\end{tabular}




\begin{tabular}{|c|c|c|c|c|c|}
\hline 65 & 1 & 0 & 3. 391839 & 4. 208442 & -4.092205 \\
\hline 66 & 16 & 0 & 3. 398047 & -1.010594 & -0.517284 \\
\hline 67 & 8 & 0 & 3. 842269 & 0.064408 & 0.596364 \\
\hline 68 & 46 & 0 & -0.255846 & 0.512130 & 0.928824 \\
\hline 69 & 1 & 0 & 0.516135 & 1. 852186 & 1. 055893 \\
\hline 70 & 6 & 0 & 4. 976925 & -1.189645 & -1.774694 \\
\hline 71 & 9 & 0 & 4. 667758 & -2.135979 & -2.726584 \\
\hline 72 & 9 & 0 & 5. 222542 & 0.020808 & -2.374936 \\
\hline 73 & 9 & 0 & 6. 073171 & -1.590735 & -1.046163 \\
\hline 74 & 6 & 0 & 0.349531 & -1.850257 & 3. 280012 \\
\hline 75 & 1 & 0 & 0.594631 & -1.891430 & 4. 351845 \\
\hline 76 & 1 & 0 & -0.666656 & -2.244200 & 3. 165791 \\
\hline 77 & 6 & 0 & 1. 335228 & -2.752408 & 2. 485474 \\
\hline 78 & 1 & 0 & 1. 031166 & -2.724116 & 1. 432868 \\
\hline 79 & 1 & 0 & 1. 200831 & -3.788232 & 2. 827060 \\
\hline 80 & 6 & 0 & 2. 812219 & -2.327821 & 2. 629096 \\
\hline 81 & 1 & 0 & 2. 896095 & -1.236298 & 2. 598683 \\
\hline 82 & 1 & 0 & 3. 202795 & -2.646471 & 3. 606293 \\
\hline 83 & 6 & 0 & 3. 743866 & -2.912845 & 1. 543298 \\
\hline 84 & 1 & 0 & 4. 764389 & -2.542308 & 1. 684489 \\
\hline 85 & 1 & 0 & 3. 785726 & -4.009309 & 1. 597067 \\
\hline 86 & 8 & 0 & 2. 110313 & -0.794836 & -1.471095 \\
\hline 87 & 7 & 0 & 3. 337043 & -2.641825 & 0.136725 \\
\hline 88 & 1 & 0 & 2. 520148 & -3.132060 & -0.237753 \\
\hline 89 & 6 & 0 & 1. 513159 & 1. 942361 & 3. 490242 \\
\hline 90 & 6 & 0 & 0.913820 & 2. 686377 & 4. 535851 \\
\hline 91 & 6 & 0 & 2. 772589 & 2. 339782 & 2. 973758 \\
\hline 92 & 6 & 0 & 1. 571888 & 3. 809308 & 5. 065520 \\
\hline 93 & 1 & 0 & -0.053018 & 2. 381686 & 4. 928411 \\
\hline 94 & 6 & 0 & 3. 421755 & 3. 462352 & 3. 510493 \\
\hline 95 & 1 & 0 & 3. 226231 & 1. 770672 & 2. 165823 \\
\hline 96 & 6 & 0 & 2. 825467 & 4. 200025 & 4. 554746 \\
\hline 97 & 1 & 0 & 1. 111624 & 4. 375106 & 5.871017 \\
\hline 98 & 1 & 0 & 4. 389637 & 3. 761754 & 3. 117653 \\
\hline 99 & 1 & 0 & 3. 332584 & 5. 069329 & 4. 965150 \\
\hline
\end{tabular}




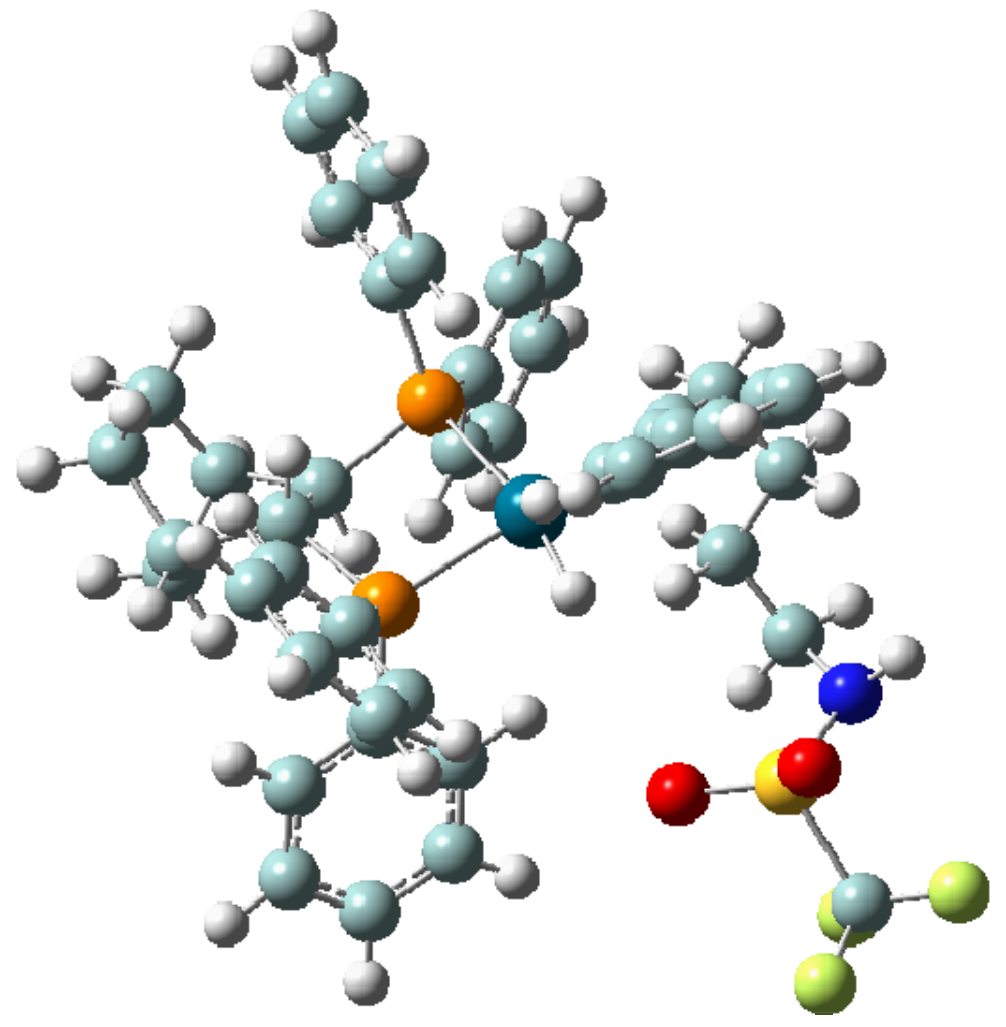

\section{Acetylenic intermediate of hydroamination, isomer II}

$\mathrm{E}(\mathrm{RB}+\mathrm{HF}-\mathrm{LYP})=-3416.54486985 \quad$ A. U.

Standard orientation:

\begin{tabular}{|c|c|c|c|c|c|}
\hline \multirow{2}{*}{$\begin{array}{l}\text { Center } \\
\text { Number }\end{array}$} & \multirow{2}{*}{$\begin{array}{l}\text { Atomic } \\
\text { Number }\end{array}$} & \multirow{2}{*}{$\begin{array}{c}\text { Atomic } \\
\text { Type }\end{array}$} & \multicolumn{3}{|c|}{ Coordinates (Angstroms) } \\
\hline & & & X & Y & Z \\
\hline 1 & 6 & 0 & 4. 833003 & -1.004503 & -2.469405 \\
\hline 2 & 6 & 0 & 3. 364731 & -1.397906 & -2.819760 \\
\hline 3 & 6 & 0 & 3.387755 & 0.883792 & -3.043868 \\
\hline 4 & 6 & 0 & 4. 873241 & 0.550498 & -2.711485 \\
\hline 5 & 1 & 0 & 5. 110426 & -1.268503 & -1.447020 \\
\hline 6 & 1 & 0 & 5.525424 & -1.525312 & -3.140307 \\
\hline 7 & 1 & 0 & 5.247018 & 1. 101627 & -1.839986 \\
\hline
\end{tabular}




\begin{tabular}{|c|c|c|c|c|c|}
\hline 8 & 1 & 0 & 5. 517559 & 0.803219 & -3.561535 \\
\hline 9 & 6 & 0 & 3. 019910 & -0.346125 & -3.925797 \\
\hline 10 & 1 & 0 & 1. 974053 & -0.372893 & -4.248958 \\
\hline 11 & 1 & 0 & 3. 663052 & -0.427881 & -4.808797 \\
\hline 12 & 6 & 0 & 2. 339486 & -0.912557 & -1.753188 \\
\hline 13 & 1 & 0 & 1. 348599 & -1.079759 & -2.203199 \\
\hline 14 & 6 & 0 & 2. 597589 & 0.628682 & -1.711866 \\
\hline 15 & 1 & 0 & 3. 249738 & 0.873236 & -0.862825 \\
\hline 16 & 1 & 0 & 3. 236302 & -2.446658 & -3.100050 \\
\hline 17 & 1 & 0 & 3. 241327 & 1. 869238 & -3.491287 \\
\hline 18 & 15 & 0 & 0.971533 & 1. 485508 & -1.244186 \\
\hline 19 & 15 & 0 & 2. 030160 & -1.551988 & 0.015227 \\
\hline 20 & 6 & 0 & -0.234574 & 0.322490 & 2. 892314 \\
\hline 21 & 6 & 0 & -0.400335 & -0.874478 & 2. 555569 \\
\hline 22 & 6 & 0 & 1. 607060 & -3.365475 & -0.190524 \\
\hline 23 & 6 & 0 & 1. 923634 & -4.296884 & 0.824276 \\
\hline 24 & 6 & 0 & 0.894426 & -3.807201 & -1.328172 \\
\hline 25 & 6 & 0 & 1. 548412 & -5.647255 & 0.695052 \\
\hline 26 & 1 & 0 & 2. 470513 & -3.981636 & 1. 709031 \\
\hline 27 & 6 & 0 & 0.520976 & -5.157787 & -1.455539 \\
\hline 28 & 1 & 0 & 0.624973 & -3.116393 & -2.122565 \\
\hline 29 & 6 & 0 & 0.847056 & -6.083370 & -0.445412 \\
\hline 30 & 1 & 0 & 1. 807387 & -6.353563 & 1. 479512 \\
\hline 31 & 1 & 0 & -0.017378 & -5.484238 & -2.341539 \\
\hline 32 & 1 & 0 & 0.562978 & -7.127285 & -0.547323 \\
\hline 33 & 6 & 0 & 3. 615028 & -1.502794 & 1. 004749 \\
\hline 34 & 6 & 0 & 3. 760227 & -0.483902 & 1. 971745 \\
\hline 35 & 6 & 0 & 4. 657795 & -2.439031 & 0.820701 \\
\hline 36 & 6 & 0 & 4. 939016 & -0.392681 & 2. 736603 \\
\hline 37 & 1 & 0 & 2. 953754 & 0.225456 & 2. 142133 \\
\hline 38 & 6 & 0 & 5. 830946 & -2.350404 & 1.590256 \\
\hline 39 & 1 & 0 & 4. 558102 & -3.237339 & 0.090074 \\
\hline 40 & 6 & 0 & 5. 975932 & -1.324971 & 2. 547454 \\
\hline 41 & 1 & 0 & 5. 039956 & 0.394061 & 3. 479489 \\
\hline 42 & 1 & 0 & 6. 627331 & -3.075237 & 1. 443650 \\
\hline 43 & 1 & 0 & 6. 883696 & -1.259617 & 3. 141423 \\
\hline 44 & 6 & 0 & -0.183731 & 1. 466009 & -2.703165 \\
\hline 45 & 6 & 0 & -1.393551 & 0.748420 & -2.622985 \\
\hline 46 & 6 & 0 & 0.137250 & 2. 179509 & -3.880977 \\
\hline 47 & 6 & 0 & -2.278566 & 0.733552 & -3.717968 \\
\hline 48 & 1 & 0 & -1.680746 & 0.233786 & -1.711251 \\
\hline 49 & 6 & 0 & -0.743655 & 2. 154137 & -4.975135 \\
\hline 50 & 1 & 0 & 1. 047671 & 2. 770208 & -3.940418 \\
\hline 51 & 6 & 0 & -1.951072 & 1. 427836 & -4.896609 \\
\hline 52 & 1 & 0 & -3.218864 & 0. 199466 & -3.625392 \\
\hline 53 & 1 & 0 & -0.497279 & 2. 706052 & -5.878359 \\
\hline 54 & 1 & 0 & -2.634636 & 1. 420212 & -5.741679 \\
\hline 55 & 6 & 0 & 1. 370337 & 3. 282359 & -0.938377 \\
\hline 56 & 6 & 0 & 2. 698736 & 3. 745286 & -0.834864 \\
\hline 57 & 6 & 0 & 0.299905 & 4. 191119 & -0.783962 \\
\hline 58 & 6 & 0 & 2. 953438 & 5. 107939 & -0.585593 \\
\hline 59 & 1 & 0 & 3. 540380 & 3. 068828 & -0.951949 \\
\hline
\end{tabular}




\begin{tabular}{|c|c|c|c|c|c|}
\hline 60 & 6 & 0 & 0.557772 & 5.550481 & -0.538090 \\
\hline 61 & 1 & 0 & -0.729186 & 3. 847256 & -0.860154 \\
\hline 62 & 6 & 0 & 1. 885955 & 6.012707 & -0.437581 \\
\hline 63 & 1 & 0 & 3. 979766 & 5. 457890 & -0.512673 \\
\hline 64 & 1 & 0 & -0.271907 & 6. 243221 & -0.426681 \\
\hline 65 & 1 & 0 & 2. 084594 & 7. 064443 & -0.249319 \\
\hline 66 & 16 & 0 & -4.941650 & -0.217927 & 0.000753 \\
\hline 67 & 8 & 0 & -4.041309 & -0.376700 & -1.329703 \\
\hline 68 & 46 & 0 & 0.292514 & 0.221916 & 0.671680 \\
\hline 69 & 1 & 0 & -0.734393 & 1. 382081 & 0.761434 \\
\hline 70 & 6 & 0 & -6.866200 & -0.244395 & -0.636499 \\
\hline 71 & 9 & 0 & -7.690340 & -0.313195 & 0.463700 \\
\hline 72 & 9 & 0 & -7.123893 & 0.898599 & -1.352433 \\
\hline 73 & 9 & 0 & -7.044992 & -1.352706 & -1.433633 \\
\hline 74 & 6 & 0 & -0.726271 & -2.319828 & 2. 678556 \\
\hline 75 & 1 & 0 & -0.055888 & -2.927793 & 2. 061990 \\
\hline 76 & 1 & 0 & -0.541523 & -2.602152 & 3. 725738 \\
\hline 77 & 6 & 0 & -2.199861 & -2.661023 & 2. 317383 \\
\hline 78 & 1 & 0 & -2.862570 & -1.987177 & 2. 879394 \\
\hline 79 & 1 & 0 & -2.402703 & -3.682504 & 2. 670660 \\
\hline 80 & 6 & 0 & -2.488789 & -2.577680 & 0.805305 \\
\hline 81 & 1 & 0 & -2.168841 & -1.603320 & 0.409925 \\
\hline 82 & 1 & 0 & -1.892961 & -3.339307 & 0.281354 \\
\hline 83 & 6 & 0 & -3.970301 & -2.803583 & 0.437349 \\
\hline 84 & 1 & 0 & -4.077412 & -2.895144 & -0.647858 \\
\hline 85 & 1 & 0 & -4.354868 & -3.730117 & 0.884493 \\
\hline 86 & 8 & 0 & -4.796584 & 1. 032992 & 1. 004662 \\
\hline 87 & 7 & 0 & -4.903146 & -1.741117 & 0.891257 \\
\hline 88 & 1 & 0 & -5.019768 & -1.573073 & 1. 891769 \\
\hline 89 & 6 & 0 & -0.196112 & 1. 531540 & 3. 690118 \\
\hline 90 & 6 & 0 & -0.684256 & 1. 458664 & 5. 022121 \\
\hline 91 & 6 & 0 & 0.320853 & 2. 757306 & 3. 209821 \\
\hline 92 & 6 & 0 & -0.651832 & 2. 591750 & 5. 848874 \\
\hline 93 & 1 & 0 & -1.087308 & 0.521634 & 5. 397332 \\
\hline 94 & 6 & 0 & 0.347227 & 3. 887615 & 4. 042241 \\
\hline 95 & 1 & 0 & 0.688931 & 2. 822905 & 2. 190755 \\
\hline 96 & 6 & 0 & -0.136436 & 3.810476 & 5. 362580 \\
\hline 97 & 1 & 0 & -1.030312 & 2.528026 & 6. 865520 \\
\hline 98 & 1 & 0 & 0.741469 & 4. 825891 & 3. 661604 \\
\hline 99 & 1 & 0 & -0.115827 & 4. 687256 & 6. 004205 \\
\hline
\end{tabular}




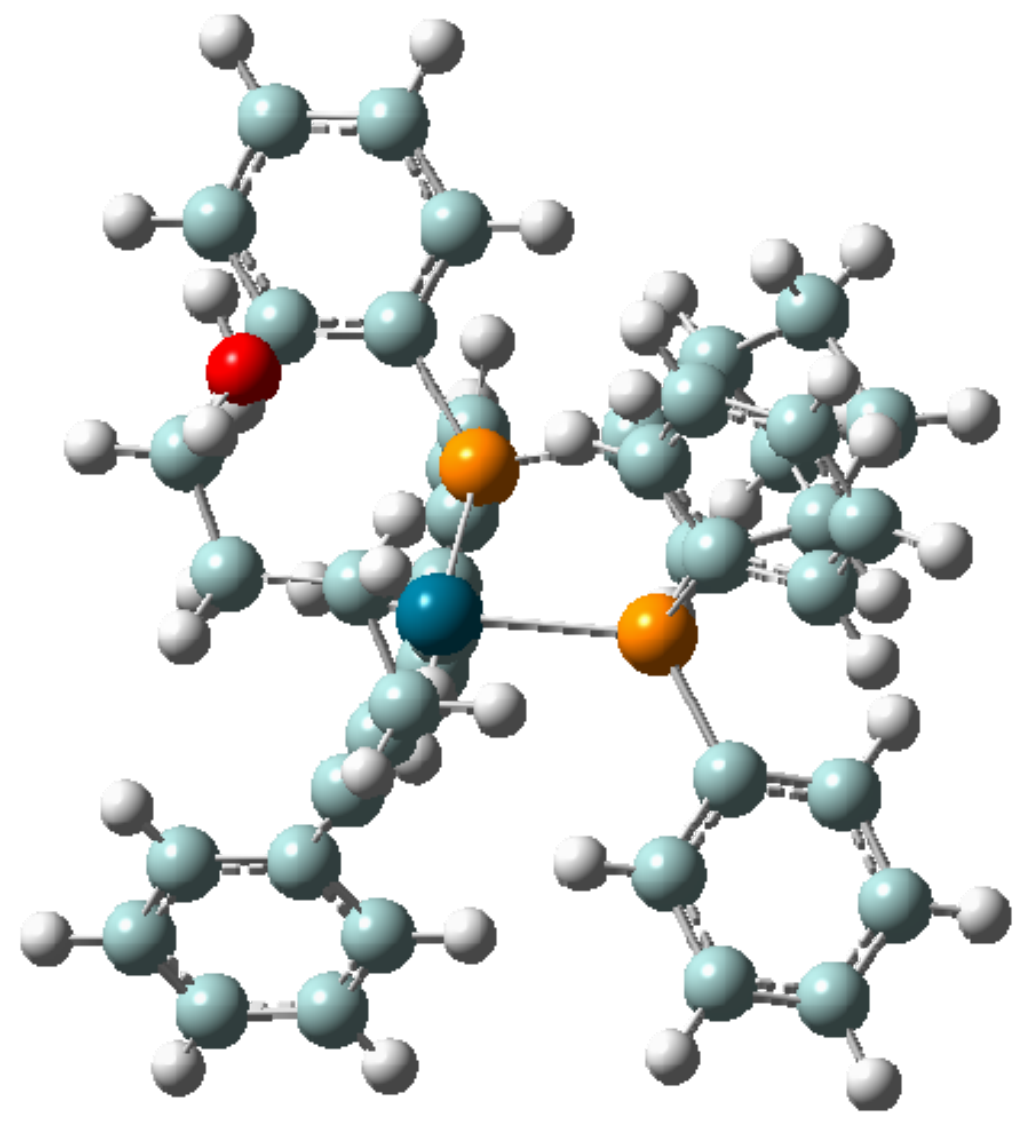

Acetylenic intermediate of hydroalkoxylation, isomer I

$\mathrm{E}(\mathrm{RB}+\mathrm{HF}-\mathrm{LYP})=-2550.99702256 \quad$ A. U.

Standard orientation:

\begin{tabular}{|c|c|c|c|c|c|}
\hline \multirow{2}{*}{$\begin{array}{l}\text { Center } \\
\text { Number }\end{array}$} & \multirow{2}{*}{$\begin{array}{l}\text { Atomic } \\
\text { Number }\end{array}$} & \multirow{2}{*}{$\begin{array}{c}\text { Atomic } \\
\text { Type }\end{array}$} & \multicolumn{3}{|c|}{ Coordinates (Angstroms) } \\
\hline & & & X & Y & Z \\
\hline 1 & 6 & 0 & -0.752320 & -3.866889 & -2.480192 \\
\hline 2 & 6 & 0 & -0.801114 & -2.395023 & -2.993845 \\
\hline 3 & 6 & 0 & 1. 407340 & -2.714942 & -2.484792 \\
\hline 4 & 6 & 0 & 0.782397 & -4.120038 & -2.228232 \\
\hline 5 & 1 & 0 & -1.350946 & -4.014877 & -1.576978 \\
\hline 6 & 1 & 0 & -1.142054 & -4.546636 & -3.246245 \\
\hline 7 & 1 & 0 & 0.984665 & -4.499062 & -1.219174 \\
\hline 8 & 1 & 0 & 1. 184472 & -4.849336 & -2.941181 \\
\hline 9 & 6 & 0 & 0.576821 & -2.262333 & -3.717809 \\
\hline
\end{tabular}




\begin{tabular}{|c|c|c|c|c|c|}
\hline 10 & 1 & 0 & 0.794479 & -1.245700 & -4.061188 \\
\hline 11 & 1 & 0 & 0.681576 & -2.950418 & -4.563932 \\
\hline 12 & 6 & 0 & -0.544617 & -1.361253 & -1.854023 \\
\hline 13 & 1 & 0 & -0.452722 & -0.384629 & -2.355300 \\
\hline 14 & 6 & 0 & 0.864810 & -1.804415 & -1.329264 \\
\hline 15 & 1 & 0 & 0.749177 & -2.420076 & -0.427938 \\
\hline 16 & 1 & 0 & -1.667789 & -2.160543 & -3.614878 \\
\hline 17 & 1 & 0 & 2. 493247 & -2.720056 & -2.602610 \\
\hline 18 & 15 & 0 & 1. 768426 & -0.272356 & -0.669923 \\
\hline 19 & 15 & 0 & -1.629302 & -0.865276 & -0.355249 \\
\hline 20 & 6 & 0 & -1.053883 & 2. 627354 & 1. 543736 \\
\hline 21 & 6 & 0 & -1.302753 & 1. 641399 & 2. 265875 \\
\hline 22 & 6 & 0 & -3.253613 & -0.237238 & -1.033751 \\
\hline 23 & 6 & 0 & -3.895517 & -0.824661 & -2.146807 \\
\hline 24 & 6 & 0 & -3.871752 & 0.852384 & -0.380302 \\
\hline 25 & 6 & 0 & -5.127968 & -0.323036 & -2.604498 \\
\hline 26 & 1 & 0 & -3.450036 & -1.671483 & -2.657407 \\
\hline 27 & 6 & 0 & -5.109627 & 1. 346446 & -0.832228 \\
\hline 28 & 1 & 0 & -3.396284 & 1. 314043 & 0.480146 \\
\hline 29 & 6 & 0 & -5.739200 & 0.762663 & -1.948030 \\
\hline 30 & 1 & 0 & -5.610319 & -0.782070 & -3.463469 \\
\hline 31 & 1 & 0 & -5.577642 & 2. 180875 & -0.316398 \\
\hline 32 & 1 & 0 & -6.693730 & 1. 145272 & -2.299474 \\
\hline 33 & 6 & 0 & -2.049036 & -2.409727 & 0.624735 \\
\hline 34 & 6 & 0 & -1.125081 & -2.853695 & 1. 598916 \\
\hline 35 & 6 & 0 & -3.252087 & -3.124044 & 0.438024 \\
\hline 36 & 6 & 0 & -1.386428 & -4.012756 & 2. 352383 \\
\hline 37 & 1 & 0 & -0.215613 & -2.288842 & 1. 791076 \\
\hline 38 & 6 & 0 & -3.516462 & -4.277032 & 1. 200546 \\
\hline 39 & 1 & 0 & -3.989044 & -2.783621 & -0.282989 \\
\hline 40 & 6 & 0 & -2.581920 & -4.729450 & 2. 152741 \\
\hline 41 & 1 & 0 & -0.668262 & -4.343435 & 3. 098210 \\
\hline 42 & 1 & 0 & -4.447848 & -4.817327 & 1. 052675 \\
\hline 43 & 1 & 0 & -2.788707 & -5.620899 & 2. 739019 \\
\hline 44 & 6 & 0 & 2. 311785 & 0.761030 & -2.124867 \\
\hline 45 & 6 & 0 & 1. 635462 & 1. 961975 & -2.422954 \\
\hline 46 & 6 & 0 & 3. 385600 & 0.337559 & -2.939277 \\
\hline 47 & 6 & 0 & 2. 019712 & 2. 728151 & -3.539140 \\
\hline 48 & 1 & 0 & 0.829793 & 2. 309071 & -1.780142 \\
\hline 49 & 6 & 0 & 3. 767959 & 1. 106996 & -4.052025 \\
\hline 50 & 1 & 0 & 3. 934532 & -0.569968 & -2.701196 \\
\hline 51 & 6 & 0 & 3. 082925 & 2. 300979 & -4.356569 \\
\hline 52 & 1 & 0 & 1. 498613 & 3. 655659 & -3.760527 \\
\hline 53 & 1 & 0 & 4. 597348 & 0.780802 & -4.673831 \\
\hline 54 & 1 & 0 & 3. 383051 & 2. 895431 & -5.215524 \\
\hline 55 & 6 & 0 & 3. 335642 & -0.861099 & 0.146858 \\
\hline 56 & 6 & 0 & 3. 613456 & -2.232232 & 0.322632 \\
\hline 57 & 6 & 0 & 4. 254887 & 0.103442 & 0.613841 \\
\hline 58 & 6 & 0 & 4. 806923 & -2.634796 & 0.952748 \\
\hline 59 & 1 & 0 & 2. 922533 & -2.994169 & -0.025631 \\
\hline 60 & 6 & 0 & 5. 446967 & -0.301124 & 1. 236826 \\
\hline 61 & 1 & 0 & 4. 046504 & 1. 164077 & 0.493289 \\
\hline
\end{tabular}




$\begin{array}{rrrrrr}62 & 6 & 0 & 5.726335 & -1.672275 & 1.408237 \\ 63 & 1 & 0 & 5.016442 & -3.693545 & 1.080607 \\ 64 & 1 & 0 & 6.151644 & 0.447529 & 1.588678 \\ 65 & 1 & 0 & 6.649314 & -1.984608 & 1.889706 \\ 66 & 46 & 0 & 0.095094 & 0.751898 & 0.665492 \\ 67 & 1 & 0 & 1.308900 & 1.590202 & 1.151804 \\ 68 & 6 & 0 & -1.858946 & 0.784750 & 3.344793 \\ 69 & 1 & 0 & -2.454839 & 1.432824 & 4.005842 \\ 70 & 1 & 0 & -2.550702 & 0.054473 & 2.902733 \\ 71 & 6 & 0 & -0.792319 & 0.027729 & 4.179103 \\ 72 & 1 & 0 & -0.230105 & -0.651050 & 3.530530 \\ 73 & 1 & 0 & -1.325978 & -0.593780 & 4.912976 \\ 74 & 6 & 0 & 0.203225 & 0.950033 & 4.907501 \\ 75 & 1 & 0 & 0.705906 & 1.603796 & 4.182597 \\ 76 & 1 & 0 & -0.327179 & 1.594553 & 5.625101 \\ 77 & 6 & 0 & 1.275315 & 0.160337 & 5.663053 \\ 78 & 1 & 0 & 1.965775 & 0.851633 & 6.169148 \\ 79 & 1 & 0 & 0.813782 & -0.496169 & 6.418298 \\ 80 & 6 & 0 & -0.890541 & 3.878606 & 0.840988 \\ 81 & 6 & 0 & -1.616911 & 4.140925 & -0.348751 \\ 82 & 6 & 0 & -0.017093 & 4.869632 & 1.356271 \\ 83 & 6 & 0 & -1.476982 & 5.376440 & -1.001265 \\ 84 & 1 & 0 & -2.288814 & 3.385781 & -0.747264 \\ 85 & 6 & 0 & 0.117057 & 6.102532 & 0.698673 \\ 86 & 1 & 0 & 0.544049 & 4.668203 & 2.264694 \\ 87 & 6 & 0 & -0.611435 & 6.359843 & -0.480766 \\ 88 & 1 & 0 & -2.044105 & 5.575339 & -1.906644 \\ 89 & 1 & 0 & 0.782648 & 6.860183 & 1.102852 \\ 90 & 1 & 0 & -0.508117 & 7.316603 & -0.985803 \\ 91 & 8 & 0 & 1.996140 & -0.647859 & 4.668983 \\ 92 & 1 & 2.790034 & -1.062874 & 5.064264 \\ --------------------------------------------------\end{array}$




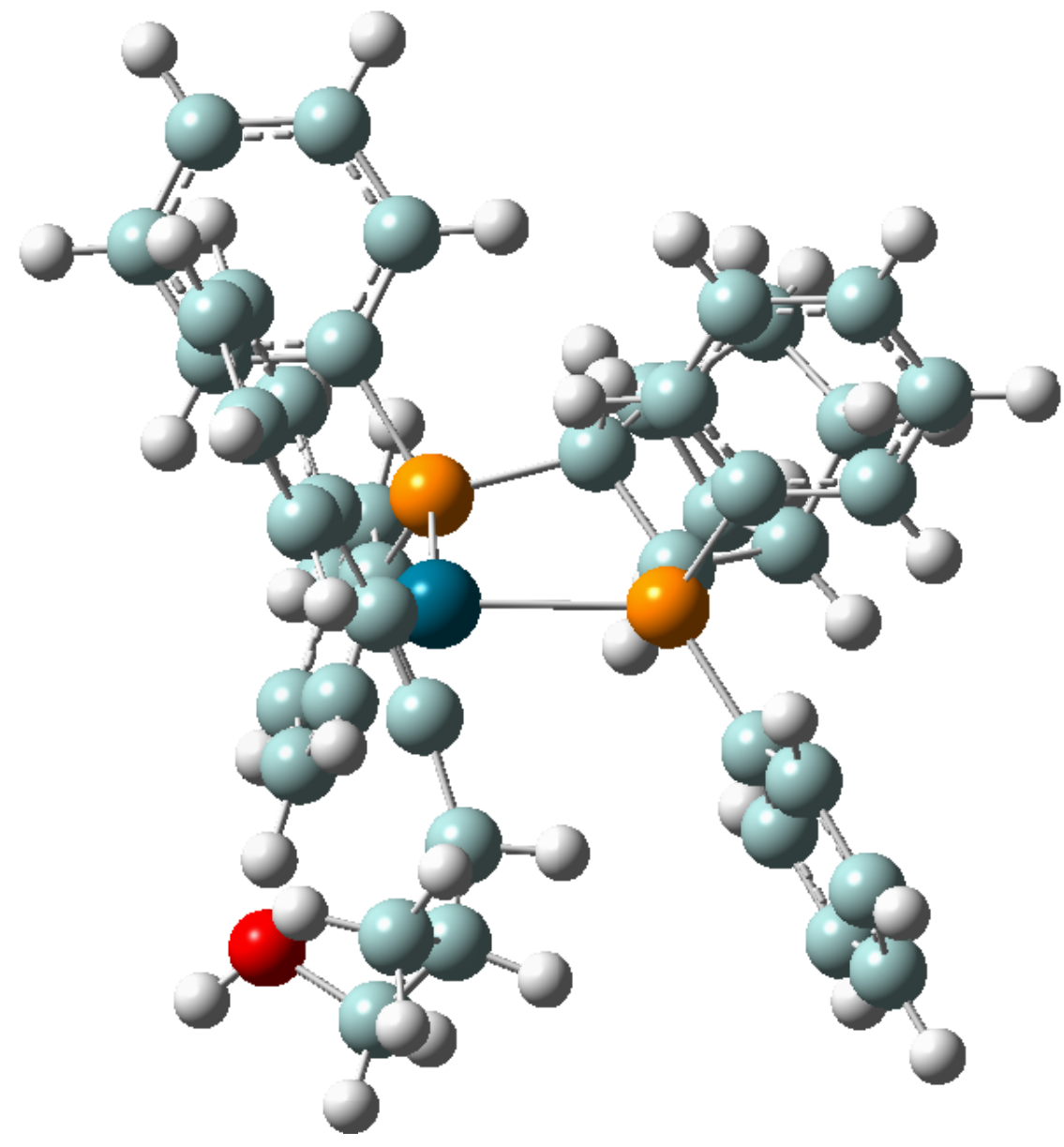

\section{Acetylenic intermediate of hydroalkoxylation, isomer II}

$\mathrm{E}(\mathrm{RB}+\mathrm{HF}-\mathrm{LYP})=-2550.99698233 \quad$ A. U.

Standard orientation:

\begin{tabular}{|c|c|c|c|c|c|}
\hline \multirow{2}{*}{$\begin{array}{l}\text { Center } \\
\text { Number }\end{array}$} & \multirow{2}{*}{$\begin{array}{l}\text { Atomic } \\
\text { Number }\end{array}$} & \multirow{2}{*}{$\begin{array}{l}\text { Atomic } \\
\text { Type }\end{array}$} & \multicolumn{3}{|c|}{ Coordinates (Angstroms) } \\
\hline & & & X & Y & Z \\
\hline 1 & 6 & 0 & -0.857903 & -1.605878 & 4. 186996 \\
\hline 2 & 6 & 0 & -1.108283 & -2.362393 & 2.845850 \\
\hline 3 & 6 & 0 & 1. 174970 & -2.214359 & 2. 972122 \\
\hline 4 & 6 & 0 & 0.710334 & -1.593825 & 4. 323437 \\
\hline 5 & 1 & 0 & -1.279232 & -0.598367 & 4. 185562 \\
\hline 6 & 1 & 0 & -1.321419 & -2.153148 & 5. 015383 \\
\hline 7 & 1 & 0 & 1. 109118 & -0.585608 & 4. 489504 \\
\hline 8 & 1 & 0 & 1. 040265 & -2.220074 & 5. 160479 \\
\hline
\end{tabular}




\begin{tabular}{|c|c|c|c|c|c|}
\hline 9 & 6 & 0 & 0.111658 & -3.340468 & 2. 806268 \\
\hline 10 & 1 & 0 & 0.194384 & -3.904689 & 1. 871131 \\
\hline 11 & 1 & 0 & 0.122953 & -4.045382 & 3. 644740 \\
\hline 12 & 6 & 0 & -0.727575 & -1.510499 & 1. 598665 \\
\hline 13 & 1 & 0 & -0.769868 & -2.203174 & 0.743020 \\
\hline 14 & 6 & 0 & 0.773488 & -1.166526 & 1. 873641 \\
\hline 15 & 1 & 0 & 0.847141 & -0.159548 & 2. 306508 \\
\hline 16 & 1 & 0 & -2.096129 & -2.824383 & 2. 773652 \\
\hline 17 & 1 & 0 & 2. 221317 & -2.526596 & 2. 959614 \\
\hline 18 & 15 & 0 & 1. 663266 & -0.955650 & 0.209589 \\
\hline 19 & 15 & 0 & -1.595365 & 0.016256 & 0.858892 \\
\hline 20 & 6 & 0 & -0.306040 & 2. 477017 & -1.945154 \\
\hline 21 & 6 & 0 & -1.254495 & 1. 743349 & -2.301228 \\
\hline 22 & 6 & 0 & -3.347897 & -0.521615 & 0.489538 \\
\hline 23 & 6 & 0 & -4.357555 & 0.456458 & 0.338844 \\
\hline 24 & 6 & 0 & -3.671192 & -1.880075 & 0.281336 \\
\hline 25 & 6 & 0 & -5.669066 & 0.079562 & -0.002902 \\
\hline 26 & 1 & 0 & -4.130891 & 1. 507976 & 0.498485 \\
\hline 27 & 6 & 0 & -4.984156 & -2.255188 & -0.060188 \\
\hline 28 & 1 & 0 & -2.917575 & -2.655849 & 0.383340 \\
\hline 29 & 6 & 0 & -5.987549 & -1.278102 & -0.203325 \\
\hline 30 & 1 & 0 & -6.437249 & 0.841335 & -0.107382 \\
\hline 31 & 1 & 0 & -5.221305 & -3.305683 & -0.207683 \\
\hline 32 & 1 & 0 & -7.001869 & -1.569388 & -0.462544 \\
\hline 33 & 6 & 0 & -1.751347 & 1. 353754 & 2. 156706 \\
\hline 34 & 6 & 0 & -0.837715 & 2. 429805 & 2. 120075 \\
\hline 35 & 6 & 0 & -2.748191 & 1. 321701 & 3. 158035 \\
\hline 36 & 6 & 0 & -0.908262 & 3. 454188 & 3. 083717 \\
\hline 37 & 1 & 0 & -0.087395 & 2. 484449 & 1. 335242 \\
\hline 38 & 6 & 0 & -2.820990 & 2. 349237 & 4. 114804 \\
\hline 39 & 1 & 0 & -3.469131 & 0.509065 & 3. 190838 \\
\hline 40 & 6 & 0 & -1.898394 & 3. 415275 & 4. 082961 \\
\hline 41 & 1 & 0 & -0.203040 & 4. 280082 & 3. 044274 \\
\hline 42 & 1 & 0 & -3.591419 & 2. 318496 & 4. 880743 \\
\hline 43 & 1 & 0 & -1.958020 & 4. 208708 & 4. 823294 \\
\hline 44 & 6 & 0 & 2. 025358 & -2.628337 & -0.527355 \\
\hline 45 & 6 & 0 & 1. 416232 & -3.008495 & -1.740930 \\
\hline 46 & 6 & 0 & 2. 922047 & -3.509607 & 0.119274 \\
\hline 47 & 6 & 0 & 1. 690300 & -4.274268 & -2.293772 \\
\hline 48 & 1 & 0 & 0.763597 & -2.332090 & -2.285533 \\
\hline 49 & 6 & 0 & 3. 188753 & -4.772246 & -0.436291 \\
\hline 50 & 1 & 0 & 3. 430417 & -3.210601 & 1. 032159 \\
\hline 51 & 6 & 0 & 2. 568253 & -5.159607 & -1.642217 \\
\hline 52 & 1 & 0 & 1. 223362 & -4.551776 & -3.234264 \\
\hline 53 & 1 & 0 & 3. 881524 & -5.445319 & 0.061744 \\
\hline 54 & 1 & 0 & 2. 780302 & -6.134967 & -2.072620 \\
\hline 55 & 6 & 0 & 3. 332534 & -0.210566 & 0.589666 \\
\hline 56 & 6 & 0 & 3. 697856 & 0.187393 & 1. 892507 \\
\hline 57 & 6 & 0 & 4. 244954 & -0.035103 & -0.474532 \\
\hline 58 & 6 & 0 & 4. 967771 & 0.749452 & 2. 128885 \\
\hline 59 & 1 & 0 & 3. 017674 & 0.062872 & 2. 729647 \\
\hline 60 & 6 & 0 & 5. 512559 & 0.521870 & -0.234795 \\
\hline
\end{tabular}




\begin{tabular}{rrrrrr}
61 & 1 & 0 & 3.974559 & -0.334858 & -1.484604 \\
62 & 6 & 0 & 5.877319 & 0.916795 & 1.068595 \\
63 & 1 & 0 & 5.243126 & 1.049327 & 3.136576 \\
64 & 1 & 0 & 6.209740 & 0.647338 & -1.058767 \\
65 & 1 & 0 & 6.857903 & 1.347155 & 1.253752 \\
66 & 46 & 0 & 0.139614 & 0.444664 & -0.982879 \\
67 & 1 & 0 & 1.317457 & 0.511979 & -1.994037 \\
68 & 6 & 0 & -2.487557 & 1.244160 & -2.959478 \\
69 & 1 & 0 & -3.231786 & 0.956112 & -2.204938 \\
70 & 1 & 0 & -2.906332 & 2.104489 & -3.502841 \\
71 & 6 & 0 & -2.275973 & 0.067128 & -3.953216 \\
72 & 1 & 0 & -1.358095 & 0.226752 & -4.530785 \\
73 & 1 & 0 & -3.115268 & 0.077317 & -4.663713 \\
74 & 6 & 0 & -2.225259 & -1.311593 & -3.265472 \\
75 & 1 & 0 & -1.492008 & -1.296432 & -2.444690 \\
76 & 1 & 0 & -3.202996 & -1.539327 & -2.816576 \\
77 & 6 & 0 & -1.838524 & -2.440927 & -4.222614 \\
78 & 1 & 0 & -2.038159 & -3.421611 & -3.766054 \\
79 & 1 & 0 & -2.413240 & -2.366792 & -5.159201 \\
80 & 6 & 0 & 0.578384 & 3.610866 & -1.773884 \\
81 & 6 & 0 & 0.158670 & 4.865708 & -2.290235 \\
82 & 6 & 0 & 1.829317 & 3.516425 & -1.119312 \\
83 & 6 & 0 & 0.979281 & 5.995547 & -2.152183 \\
84 & 1 & 0 & -0.799926 & 4.946115 & -2.795816 \\
85 & 6 & 0 & 2.645919 & 4.651195 & -0.987851 \\
86 & 1 & 0 & 2.160424 & 2.557502 & -0.732593 \\
87 & 6 & 0 & 2.225565 & 5.893572 & -1.500715 \\
88 & 1 & 0 & 0.651550 & 6.951088 & -2.552809 \\
89 & 1 & 0 & 3.608151 & 4.565854 & -0.490074 \\
90 & 1 & 0 & 2.859464 & 6.770219 & -1.398043 \\
91 & 8 & 0 & -0.397352 & -2.306960 & -4.490177 \\
92 & 1 & -0.139635 & -2.771218 & -5.313108 \\
--------------------------------------------------------- \\
& 1 & & & & \\
\hline 5 & & 0 & 0 & 0 &
\end{tabular}




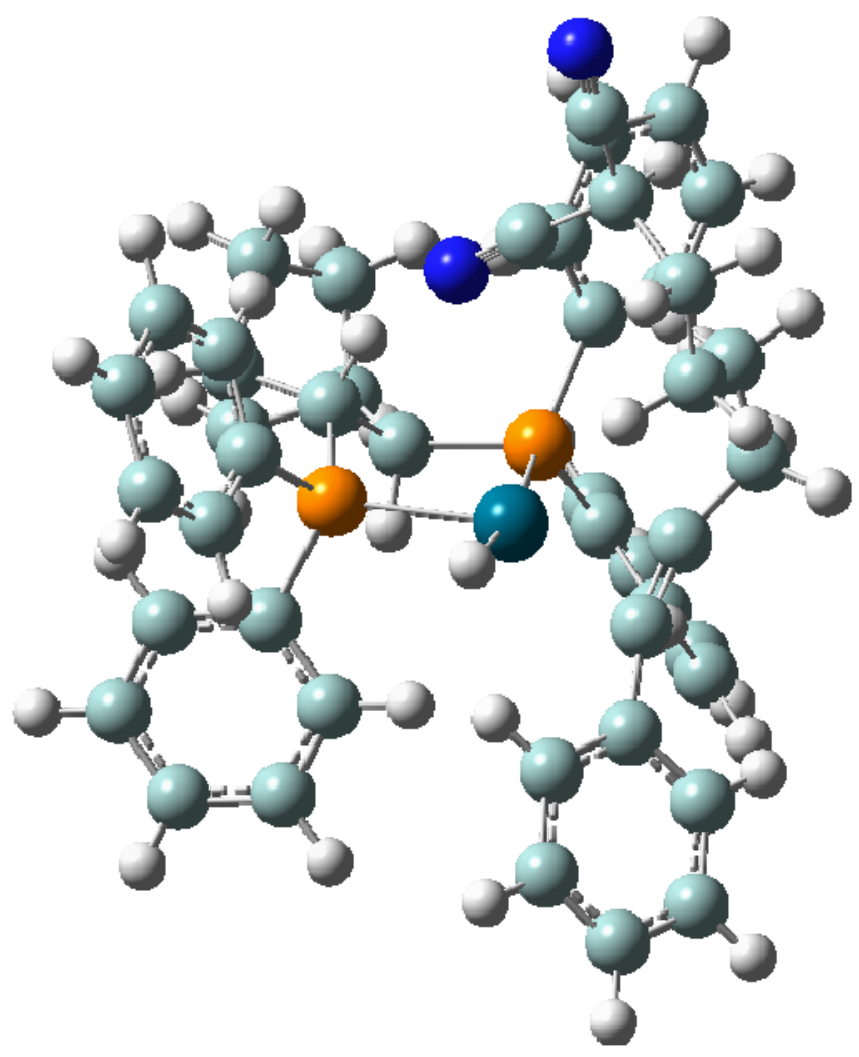

\section{Acetylenic intermediate of hydrocarbonation, isomer I}

$\mathrm{E}(\mathrm{RB}+\mathrm{HF}-\mathrm{LYP})=-2699.51423298 \quad$ A. U.

Standard orientation:

\begin{tabular}{|c|c|c|c|c|c|}
\hline \multirow{2}{*}{$\begin{array}{l}\text { Center } \\
\text { Number }\end{array}$} & \multirow{2}{*}{$\begin{array}{l}\text { Atomic } \\
\text { Number }\end{array}$} & \multirow{2}{*}{$\begin{array}{l}\text { Atomic } \\
\text { Type }\end{array}$} & \multicolumn{3}{|c|}{ Coordinates (Angstroms) } \\
\hline & & & $\mathrm{X}$ & Y & Z \\
\hline 1 & 6 & 0 & 0.965157 & -1.134899 & -4.445548 \\
\hline 2 & 6 & 0 & -0.484837 & -1.119742 & -3.875144 \\
\hline 3 & 6 & 0 & 0.116625 & 1. 092369 & -3.873887 \\
\hline 4 & 6 & 0 & 1. 344456 & 0.392205 & -4.534713 \\
\hline 5 & 1 & 0 & 1. 658663 & -1.701100 & -3.817333 \\
\hline 6 & 1 & 0 & 0.971486 & -1.599002 & -5.438152 \\
\hline 7 & 1 & 0 & 2.287975 & 0.622873 & -4.025580 \\
\hline 8 & 1 & 0 & 1. 446964 & 0.713106 & -5.577868 \\
\hline 9 & 6 & 0 & -1.037906 & 0.224310 & -4.453050 \\
\hline 10 & 1 & 0 & -2.034035 & 0.482563 & -4.079920 \\
\hline 11 & 1 & 0 & -1.056371 & 0.240701 & -5.548361 \\
\hline 12 & 6 & 0 & -0.525805 & -0.731148 & -2.366814 \\
\hline
\end{tabular}




\begin{tabular}{|c|c|c|c|c|c|}
\hline 13 & 1 & 0 & -1.592376 & -0.590191 & -2.131593 \\
\hline 14 & 6 & 0 & 0.173482 & 0.670304 & -2.365919 \\
\hline 15 & 1 & 0 & 1. 225349 & 0.578468 & -2.072069 \\
\hline 16 & 1 & 0 & -1.076022 & -2.006448 & -4.113898 \\
\hline 17 & 1 & 0 & 0.065283 & 2. 168481 & -4.054014 \\
\hline 18 & 15 & 0 & -0.553143 & 1. 681155 & -0.931899 \\
\hline 19 & 15 & 0 & -0.015578 & -1.744673 & -0.830063 \\
\hline 20 & 6 & 0 & -1.003676 & -0.588931 & 2. 963134 \\
\hline 21 & 6 & 0 & 0.064322 & -1.193610 & 2. 716541 \\
\hline 22 & 6 & 0 & -1.265955 & -3.133192 & -0.786485 \\
\hline 23 & 6 & 0 & -1.482552 & -3.978041 & -1.899942 \\
\hline 24 & 6 & 0 & -2.040173 & -3.316158 & 0.379293 \\
\hline 25 & 6 & 0 & -2.458124 & -4.989245 & -1.844171 \\
\hline 26 & 1 & 0 & -0.894418 & -3.859996 & -2.805494 \\
\hline 27 & 6 & 0 & -3.014583 & -4.331035 & 0.434266 \\
\hline 28 & 1 & 0 & -1.888860 & -2.668753 & 1. 237764 \\
\hline 29 & 6 & 0 & -3.226248 & -5.168817 & -0.676279 \\
\hline 30 & 1 & 0 & -2.617103 & -5.632750 & -2.705367 \\
\hline 31 & 1 & 0 & -3.606557 & -4.462746 & 1. 336172 \\
\hline 32 & 1 & 0 & -3.979710 & -5.950909 & -0.635171 \\
\hline 33 & 6 & 0 & 1. 658962 & -2.547650 & -1.109580 \\
\hline 34 & 6 & 0 & 2. 805733 & -1.722554 & -1.184862 \\
\hline 35 & 6 & 0 & 1. 813597 & -3.947677 & -1.203453 \\
\hline 36 & 6 & 0 & 4. 077123 & -2.287271 & -1.391775 \\
\hline 37 & 1 & 0 & 2. 724451 & -0.645113 & -1.075186 \\
\hline 38 & 6 & 0 & 3. 090713 & -4.511072 & -1.392957 \\
\hline 39 & 1 & 0 & 0.953036 & -4.604586 & -1.126552 \\
\hline 40 & 6 & 0 & 4. 224771 & -3.684536 & -1.498331 \\
\hline 41 & 1 & 0 & 4. 946844 & -1.639345 & -1.471392 \\
\hline 42 & 1 & 0 & 3. 194802 & -5.590684 & -1.462822 \\
\hline 43 & 1 & 0 & 5. 207265 & -4.121078 & -1.657043 \\
\hline 44 & 6 & 0 & -2.349990 & 2. 015953 & -1.308952 \\
\hline 45 & 6 & 0 & -3.355413 & 1. 352455 & -0.575617 \\
\hline 46 & 6 & 0 & -2.710499 & 2. 915158 & -2.337746 \\
\hline 47 & 6 & 0 & -4.712487 & 1. 577871 & -0.873294 \\
\hline 48 & 1 & 0 & -3.084467 & 0.678124 & 0.233172 \\
\hline 49 & 6 & 0 & -4.066828 & 3. 138145 & -2.632501 \\
\hline 50 & 1 & 0 & -1.947339 & 3. 451996 & -2.895103 \\
\hline 51 & 6 & 0 & -5.070256 & 2. 467525 & -1.903415 \\
\hline 52 & 1 & 0 & -5.481829 & 1. 066610 & -0.300952 \\
\hline 53 & 1 & 0 & -4.339176 & 3. 832984 & -3.422377 \\
\hline 54 & 1 & 0 & -6.117955 & 2. 644215 & -2.132096 \\
\hline 55 & 6 & 0 & 0.268228 & 3. 351069 & -0.926676 \\
\hline 56 & 6 & 0 & 1. 510303 & 3. 558984 & -1.558462 \\
\hline 57 & 6 & 0 & -0.359471 & 4. 418262 & -0.247187 \\
\hline 58 & 6 & 0 & 2. 109277 & 4. 831797 & -1.529418 \\
\hline 59 & 1 & 0 & 2. 019472 & 2. 751594 & -2.073543 \\
\hline 60 & 6 & 0 & 0.245363 & 5.686008 & -0.213818 \\
\hline 61 & 1 & 0 & -1.317336 & 4. 270220 & 0.246212 \\
\hline 62 & 6 & 0 & 1. 481411 & 5. 896682 & -0.857444 \\
\hline 63 & 1 & 0 & 3. 066084 & 4. 985873 & -2.021070 \\
\hline 64 & 1 & 0 & -0.245174 & 6. 503772 & 0.307310 \\
\hline
\end{tabular}




\begin{tabular}{rrrrrr}
65 & 1 & 0 & 1.948239 & 6.877788 & -0.833544 \\
66 & 46 & 0 & -0.285131 & 0.148549 & 0.887802 \\
67 & 1 & 0 & -0.437934 & 1.469528 & 1.691529 \\
68 & 6 & 0 & 1.228380 & -2.091963 & 2.940540 \\
69 & 1 & 0 & 1.127690 & -2.486271 & 3.963335 \\
70 & 1 & 0 & 1.168288 & -2.952380 & 2.260206 \\
71 & 6 & 0 & 2.622655 & -1.429984 & 2.784780 \\
72 & 1 & 0 & 2.805356 & -1.264353 & 1.716386 \\
73 & 1 & 0 & 3.369236 & -2.159798 & 3.131697 \\
74 & 6 & 0 & 2.766225 & -0.104741 & 3.564106 \\
75 & 1 & 0 & 2.079664 & 0.638044 & 3.137275 \\
76 & 1 & 0 & 2.452559 & -0.253909 & 4.607348 \\
77 & 6 & 0 & 4.197956 & 0.476163 & 3.592237 \\
78 & 1 & 0 & 4.177280 & 1.477700 & 4.036575 \\
79 & 1 & 0 & 4.854896 & -0.142885 & 4.216298 \\
80 & 6 & 0 & -2.249312 & -0.158088 & 3.564896 \\
81 & 6 & 0 & -3.089794 & -1.150873 & 4.135502 \\
82 & 6 & 0 & -2.632535 & 1.202263 & 3.648353 \\
83 & 6 & 0 & -4.278152 & -0.782672 & 4.786889 \\
84 & 1 & 0 & -2.800120 & -2.197127 & 4.079673 \\
85 & 6 & 0 & -3.820446 & 1.562454 & 4.303058 \\
86 & 1 & 0 & -2.000147 & 1.965253 & 3.204429 \\
87 & 6 & 0 & -4.647560 & 0.574209 & 4.873898 \\
88 & 1 & 0 & -4.910674 & -1.548512 & 5.227607 \\
89 & 1 & 0 & -4.101559 & 2.609974 & 4.368655 \\
90 & 1 & 0 & -5.566866 & 0.857412 & 5.379275 \\
91 & 6 & 0 & 4.884147 & 0.566315 & 2.179558 \\
92 & 1 & 0 & 5.140542 & -0.453865 & 1.857509 \\
93 & 6 & 0 & 6.142215 & 1.339325 & 2.242296 \\
94 & 6 & 0 & 3.978044 & 1.113474 & 1.144715 \\
95 & 7 & 0 & 7.152442 & 1.942241 & 2.317898 \\
96 & 7 & 3.209301 & 1.464566 & 0.326232 \\
------------------------------------------------------- \\
\hline 9 & & & & & \\
\hline 9 & & 0 & 0 & 0 & 0
\end{tabular}




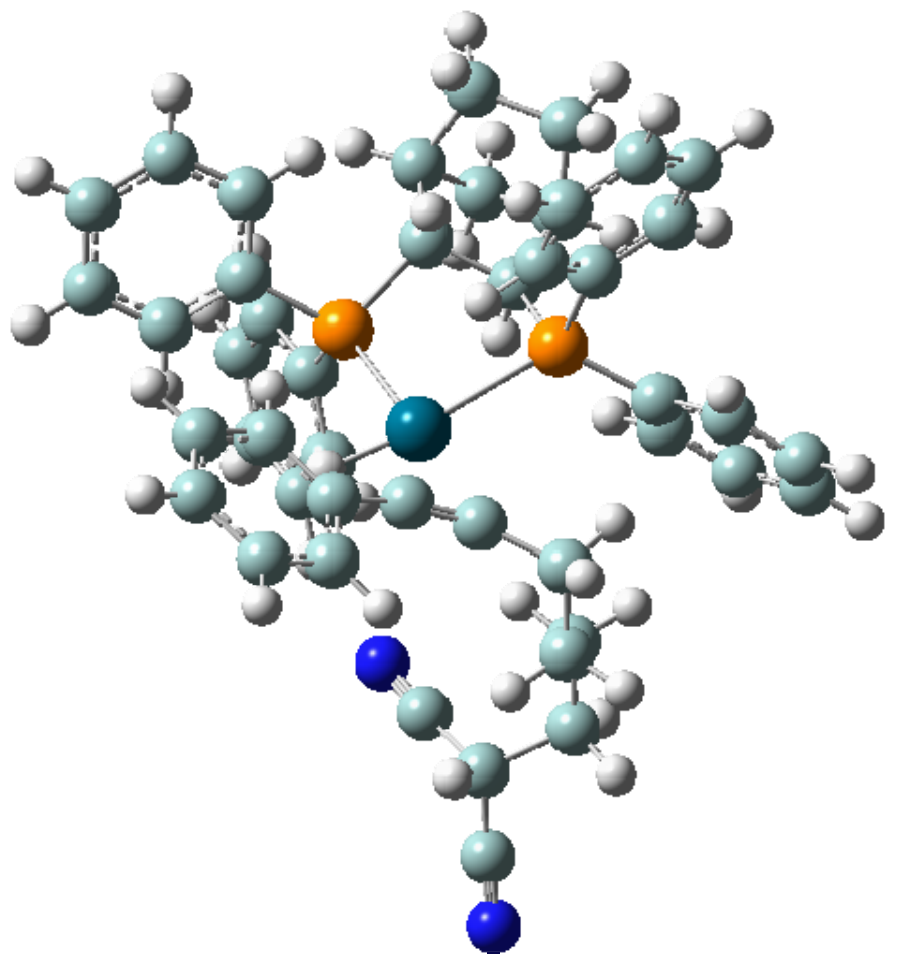

\section{Acetylenic intermediate of hydrocarbonation, isomer II}

$\mathrm{E}(\mathrm{RB}+\mathrm{HF}-\mathrm{LYP})=-2699.51459021 \quad$ A. U.

Standard orientation:

\begin{tabular}{rrrrrr} 
Center & Atomic & Atomic & \multicolumn{3}{c}{ Coordinates (Angstroms) } \\
Number & Number & Type & X & Y \\
-1 & 6 & 0 & 2.659904 & 4.076094 & 1.104204 \\
2 & 6 & 0 & 1.427150 & 3.457141 & 1.831813 \\
3 & 6 & 0 & 3.105780 & 1.942947 & 2.215956 \\
4 & 6 & 0 & 3.834256 & 3.084763 & 1.445573 \\
5 & 1 & 0 & 2.508474 & 4.179447 & 0.027971 \\
6 & 1 & 0 & 2.860822 & 5.076640 & 1.503245 \\
7 & 1 & 0 & 4.356116 & 2.727428 & 0.549539 \\
8 & 1 & 0 & 4.580278 & 3.561026 & 2.092205 \\
9 & 6 & 0 & 2.106669 & 2.780255 & 3.068534 \\
10 & 1 & 0 & 1.419781 & 2.182156 & 3.676547
\end{tabular}




\begin{tabular}{|c|c|c|c|c|c|}
\hline 11 & 1 & 0 & 2. 619675 & 3. 496537 & 3. 719482 \\
\hline 12 & 6 & 0 & 0.913397 & 2. 155464 & 1. 148068 \\
\hline 13 & 1 & 0 & 0.187643 & 1. 724229 & 1. 854488 \\
\hline 14 & 6 & 0 & 2. 185396 & 1. 247057 & 1. 151351 \\
\hline 15 & 1 & 0 & 2. 681022 & 1. 297406 & 0.172833 \\
\hline 16 & 1 & 0 & 0.632438 & 4. 173566 & 2. 055673 \\
\hline 17 & 1 & 0 & 3. 776969 & 1. 275171 & 2. 760065 \\
\hline 18 & 15 & 0 & 1. 619336 & -0.561539 & 1. 236793 \\
\hline 19 & 15 & 0 & -0.027786 & 1. 936250 & -0.494096 \\
\hline 20 & 6 & 0 & -0.677934 & -1.735141 & -2.364891 \\
\hline 21 & 6 & 0 & -1.550896 & -0.848581 & -2.185298 \\
\hline 22 & 6 & 0 & -1.570772 & 2. 980926 & -0.306188 \\
\hline 23 & 6 & 0 & -2.172292 & 3. 582416 & -1.434717 \\
\hline 24 & 6 & 0 & -2.190379 & 3. 118773 & 0.956137 \\
\hline 25 & 6 & 0 & -3.363625 & 4. 319591 & -1.299752 \\
\hline 26 & 1 & 0 & -1.711966 & 3. 495498 & -2.415523 \\
\hline 27 & 6 & 0 & -3.380623 & 3. 857116 & 1. 089201 \\
\hline 28 & 1 & 0 & -1.760893 & 2. 661487 & 1. 843336 \\
\hline 29 & 6 & 0 & -3.971963 & 4. 460834 & -0.037612 \\
\hline 30 & 1 & 0 & -3.809159 & 4. 784661 & -2.175262 \\
\hline 31 & 1 & 0 & -3.839089 & 3. 963683 & 2. 068865 \\
\hline 32 & 1 & 0 & -4.888187 & 5. 035585 & 0.067118 \\
\hline 33 & 6 & 0 & 0.937430 & 2. 696211 & -1.902601 \\
\hline 34 & 6 & 0 & 1. 587472 & 1. 824859 & -2.803761 \\
\hline 35 & 6 & 0 & 1. 033728 & 4. 093300 & -2.095253 \\
\hline 36 & 6 & 0 & 2. 337427 & 2. 342443 & -3.877260 \\
\hline 37 & 1 & 0 & 1. 496782 & 0.748291 & -2.680419 \\
\hline 38 & 6 & 0 & 1. 777677 & 4. 607850 & -3.171483 \\
\hline 39 & 1 & 0 & 0.529025 & 4. 778812 & -1.419557 \\
\hline 40 & 6 & 0 & 2. 434524 & 3. 733968 & -4.062452 \\
\hline 41 & 1 & 0 & 2. 832474 & 1. 663432 & -4.566374 \\
\hline 42 & 1 & 0 & 1. 845916 & 5. 683088 & -3.313697 \\
\hline 43 & 1 & 0 & 3. 008196 & 4. 135079 & -4.893759 \\
\hline 44 & 6 & 0 & 1. 030274 & -0.963069 & 2. 955182 \\
\hline 45 & 6 & 0 & -0.316659 & -1.323119 & 3. 159822 \\
\hline 46 & 6 & 0 & 1. 926626 & -0.936213 & 4. 048235 \\
\hline 47 & 6 & 0 & -0.774134 & -1.649260 & 4. 450592 \\
\hline 48 & 1 & 0 & -1.009617 & -1.384796 & 2. 326194 \\
\hline 49 & 6 & 0 & 1. 465050 & -1.251831 & 5. 337320 \\
\hline 50 & 1 & 0 & 2. 976540 & -0.698339 & 3. 898926 \\
\hline 51 & 6 & 0 & 0.114173 & -1.605684 & 5. 540383 \\
\hline 52 & 1 & 0 & -1.809470 & -1.946694 & 4. 577206 \\
\hline 53 & 1 & 0 & 2. 155706 & -1.233335 & 6. 176226 \\
\hline 54 & 1 & 0 & -0.234926 & -1.859394 & 6.537979 \\
\hline 55 & 6 & 0 & 3. 136077 & -1.615061 & 0.965188 \\
\hline 56 & 6 & 0 & 4. 335063 & -1.074213 & 0.455479 \\
\hline 57 & 6 & 0 & 3. 063447 & -2.994163 & 1. 261904 \\
\hline 58 & 6 & 0 & 5. 453607 & -1.905068 & 0.251083 \\
\hline 59 & 1 & 0 & 4. 419447 & -0.016996 & 0.221882 \\
\hline 60 & 6 & 0 & 4. 182291 & -3.820186 & 1. 058755 \\
\hline 61 & 1 & 0 & 2. 146462 & -3.423549 & 1. 658936 \\
\hline 62 & 6 & 0 & 5. 380851 & -3.277773 & 0.552167 \\
\hline
\end{tabular}




\begin{tabular}{|c|c|c|c|c|c|}
\hline 63 & 1 & 0 & 6. 375521 & -1.479122 & -0.135826 \\
\hline 64 & 1 & 0 & 4. 119766 & -4.878333 & 1. 297836 \\
\hline 65 & 1 & 0 & 6.246589 & -3.916431 & 0.398636 \\
\hline 66 & 46 & 0 & -0.048399 & -0.627642 & -0.483591 \\
\hline 67 & 1 & 0 & 0.073401 & -2.139886 & -0.152412 \\
\hline 68 & 6 & 0 & -2.755922 & -0.035451 & -2.505302 \\
\hline 69 & 1 & 0 & -2.588717 & 1. 021530 & -2.271292 \\
\hline 70 & 1 & 0 & -2.897258 & -0.102771 & -3.594329 \\
\hline 71 & 6 & 0 & -4.060073 & -0.511564 & -1.801008 \\
\hline 72 & 1 & 0 & -4.121850 & -1.606910 & -1.868438 \\
\hline 73 & 1 & 0 & -4.910315 & -0.110213 & -2.370793 \\
\hline 74 & 6 & 0 & -4.175667 & -0.032195 & -0.338297 \\
\hline 75 & 1 & 0 & -3.290945 & -0.347001 & 0. 233924 \\
\hline 76 & 1 & 0 & -4.168855 & 1. 065660 & -0.330483 \\
\hline 77 & 6 & 0 & -5.463721 & -0.506220 & 0.372256 \\
\hline 78 & 1 & 0 & -5.668774 & 0.119433 & 1. 248562 \\
\hline 79 & 1 & 0 & -6.326601 & -0.414858 & -0.299705 \\
\hline 80 & 6 & 0 & 0.031970 & -2.854045 & -2.954328 \\
\hline 81 & 6 & 0 & -0.615714 & -3.575624 & -3.992464 \\
\hline 82 & 6 & 0 & 1. 339205 & -3.228177 & -2.564389 \\
\hline 83 & 6 & 0 & 0.037995 & -4.645942 & -4.622159 \\
\hline 84 & 1 & 0 & -1.621585 & -3.297231 & -4.295861 \\
\hline 85 & 6 & 0 & 1. 985755 & -4.301815 & -3.197150 \\
\hline 86 & 1 & 0 & 1. 839275 & -2.682451 & -1.770196 \\
\hline 87 & 6 & 0 & 1. 340293 & -5.013452 & -4.227126 \\
\hline 88 & 1 & 0 & -0.465221 & -5.194032 & -5.414138 \\
\hline 89 & 1 & 0 & 2. 989053 & -4.582325 & -2.888451 \\
\hline 90 & 1 & 0 & 1. 842759 & -5.844229 & -4.715141 \\
\hline 91 & 6 & 0 & -5.394520 & -2.000772 & 0.852512 \\
\hline 92 & 1 & 0 & -5.152428 & -2.644419 & -0.005577 \\
\hline 93 & 6 & 0 & -4.317932 & -2.161813 & 1. 855262 \\
\hline 94 & 6 & 0 & -6.692562 & -2.451260 & 1. 399327 \\
\hline 95 & 7 & 0 & -3.427407 & -2.215985 & 2. 622332 \\
\hline 96 & 7 & 0 & -7.743985 & -2.792808 & 1. 808726 \\
\hline
\end{tabular}

Full reference 41:

41. Gaussian 03, Revision B.05,M. J. Frisch, G. W. Trucks, H. B. Schlegel, G. E. Scuseria, M. A. Robb, J. R. Cheeseman, J. A. Montgomery, Jr., T. Vreven, K. N. Kudin, J. C. Burant, J. M. Millam, S. S. Iyengar, J. Tomasi, V. Barone, B. Mennucci, M. Cossi, G. Scalmani, N. Rega, G. A. Petersson, H. Nakatsuji, M. Hada, M. Ehara, K. Toyota, R. Fukuda, J. Hasegawa, M. Ishida, T. Nakajima, Y. Honda, O. Kitao, H. Nakai, M. Klene, X. Li, J. E. Knox, H. P. Hratchian, J. B. Cross, C. Adamo, J. Jaramillo, R. Gomperts, R. E. Stratmann, O. Yazyev, A. J. Austin, R. Cammi, C. Pomelli, J. W. Ochterski, P. Y. Ayala, K. Morokuma, G. A. Voth, P. Salvador, J. J. Dannenberg, V. 
G. Zakrzewski, S. Dapprich, A. D. Daniels, M. C. Strain, O. Farkas, D. K. Malick, A. D. Rabuck, K. Raghavachari, J. B. Foresman, J. V. Ortiz, Q. Cui, A. G. Baboul, S. Clifford, J. Cioslowski, B. B. Stefanov, G. Liu, A. Liashenko, P. Piskorz, I. Komaromi, R. L. Martin, D. J. Fox, T. Keith, M. A. Al-Laham, C. Y. Peng, A. Nanayakkara, M. Challacombe, P. M. W. Gill, B. Johnson, W. Chen, M. W. Wong, C. Gonzalez, and J. A. Pople, Gaussian, Inc., Pittsburgh PA, 2003. 